\title{
DEVELOPMENT OF A STATISTICAL SHAPE-FUNCTION MODEL OF THE \\ IMPLANTED KNEE FOR REAL-TIME PREDICTION OF JOINT MECHANICS
}

by

Kalin Gibbons

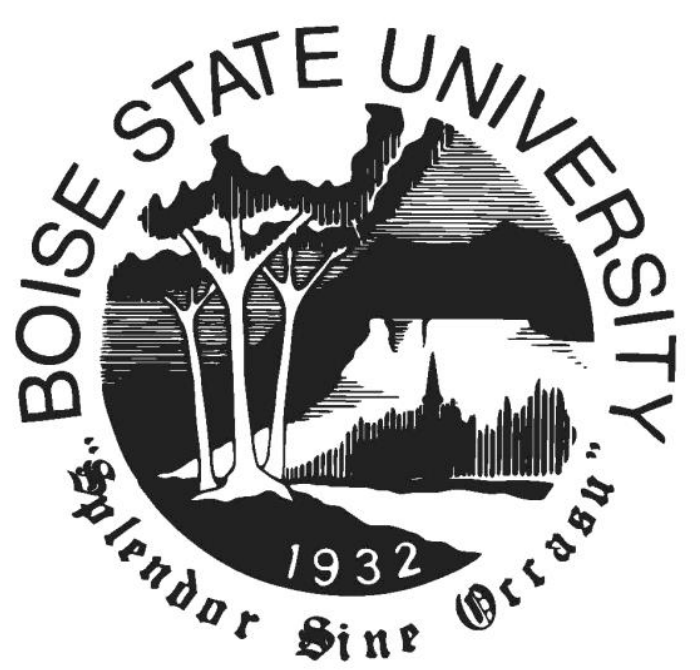

\author{
A thesis \\ submitted in partial fulfillment \\ of the requirements for the degree of \\ Master of Science in Mechanical Engineering \\ Boise State University
}

August 2019 
(C) 2019

Kalin Gibbons

ALL RIGHTS RESERVED 


\title{
BOISE STATE UNIVERSITY GRADUATE COLLEGE
}

\section{DEFENSE COMMITTEE AND FINAL READING APPROVALS}

\author{
of the thesis submitted by
}

\author{
Kalin Gibbons
}

Thesis Title: Development of a Statistical Shape-function Model of the Implanted Knee for Real-time Prediction of Joint Mechanics

Date of Final Oral Examination: $\quad 07$ June 2019

The following individuals read and discussed the thesis submitted by student Kalin Gibbons, and they evaluated his presentation and response to questions during the final oral examination. They found that the student passed the final oral examination.

Clare Fitzpatrick, Ph.D.

Trevor Lujan, Ph.D.

Gunes Uzer, Ph.D.
Chair, Supervisory Committee

Member, Supervisory Committee

Member, Supervisory Committee

The final reading approval of the thesis was granted by Clare Fitzpatrick, Ph.D., Chair of the Supervisory Committee. The thesis was approved for the Graduate College. 


\section{DEDICATION}

This thesis is dedicated to my family, who have supported me through a lifetime of schooling and taught me everything that cannot be learned from a book. My father, specifically, taught me to be meticulous in everything I do; to focus on doing things correctly the first time, and that speed and efficiency will follow. My mother taught me to be compassionate in every not-so-grand gesture of everyday life; a skill which pays higher dividends as I age, and will likely become most important of all. My sister, of course, who lets me live the nonacademic family life vicariously through her and her children, and never complains when her first notifications of my impending visits occur mere hours before my arrival. You all supply the light at the end of this education tunnel. 


\section{ACKNOWLEDGMENTS}

Firstly, I would like to thank my advisor, Dr. Clare Fitzpatrick, for allowing me the opportunity to perform research within a field which I was wholly unfamiliar. She has a preternatural knack for leading us students into well-suited projects, demonstrating care with every nuanced word of guidance or criticisms she offers. I would be much less eager to continue onward to a $\mathrm{PhD}$ without such a wonderful advisor, and her character should be a lesson to professors the world over.

I would like to express my appreciation of Drs. Trevor Lujan and Gunes Uzer for being on my thesis committee, who are very adept at asking questions that cause me a great deal of effort to answer, but make the resulting works ten-fold more compelling. This thesis is stronger because of your guidance, and I am more prepared for the work yet to come.

Going further back, I would like to thank Dr. Krishna Pakala and Matt Schmasow, who gave me my first opportunity as a learning assistant, and taught me the interpersonal and leadership skills that have allowed me to thrive as a graduate student. Without that opportunity, undergraduate life would have continued being much lonelier. Working with these two left me fully engaged with my engineering peers and my studies, illuminating the path forward to graduate school.

Lastly, I would like to thank the Boise State Mechanical and Biomedical Engineering office and IT staff, who have always gone above and beyond to solve any problems that arise. They've all had to put up with my tendency to do things in person when an email 
would surely suffice, and I'm very cognizant of all the work they do to shield us from the bureaucratic roadblocks; us engineers have the only departments where a person can have an original thought without worrying that a magical paddle might spring forth to smack them in the face. 


\begin{abstract}
Outcomes of total knee arthroplasty (TKA) are dependent on surgical technique, patient variability, and implant design. Non-optimal design or alignment choices may result in undesirable contact mechanics and joint kinematics, including poor joint alignment, instability, and reduced range of motion. Implant design and surgical alignment are modifiable factors with potential to improve patient outcomes, and there is a need for robust implant designs that can accommodate patient variability. Our objective was to develop a statistical shape-function model (SFM) of a posterior stabilized implant knee to instantaneously predict output mechanics in an efficient manner. Finite element methods were combined with Latin hypercube sampling and regression analyses to produce modeling equations relating nine implant design and six surgical alignment parameters to tibiofemoral (TF) joint mechanics outcomes during a deep knee bend. A SFM was developed and TF contact mechanics, kinematics, and soft tissue loads were instantaneously predicted from the model. Average normalized root-mean-square prediction errors were between $2.79 \%$ and $9.42 \%$, depending on the number of parameters included in the model. The statistical shape-function model generated instantaneous joint mechanics predictions using a maximum of 130 training simulations, making it ideally suited for integration into a patient-specific design and alignment optimization pipeline. Such a tool may be used to optimize kinematic function to achieve more natural motion or minimize implant wear and may aid the engineering and clinical communities in improving patient satisfaction and surgical outcomes.
\end{abstract}




\section{TABLE OF CONTENTS}

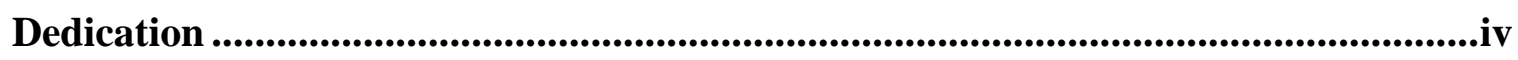

Acknowledgments...................................................................................................................v

Abstract.........................................................................................................................................vii

List of Tables ....................................................................................................................ii

List of Figures ..................................................................................................................................xiv

List of Abbreviations ......................................................................................................................

Chapter 1 Introduction ........................................................................................................1

$1.1 \quad$ The Knee

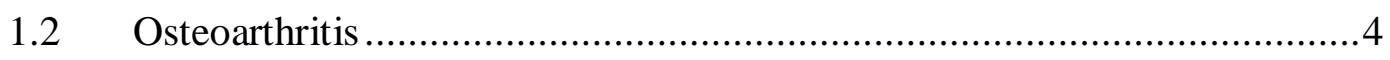

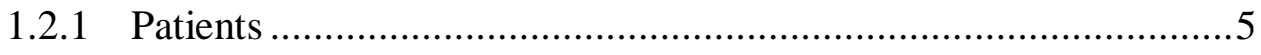

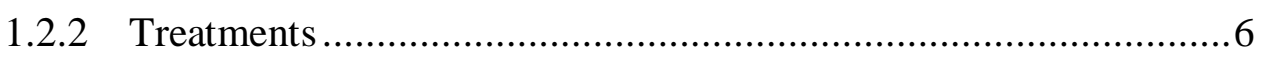

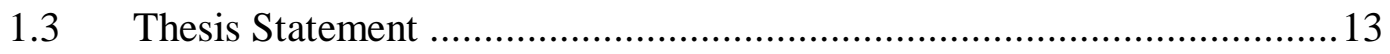

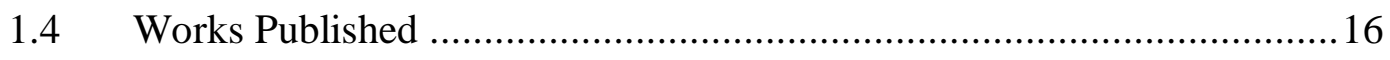

Chapter 2 Literature Review .............................................................................................17

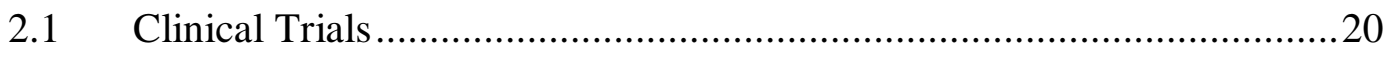

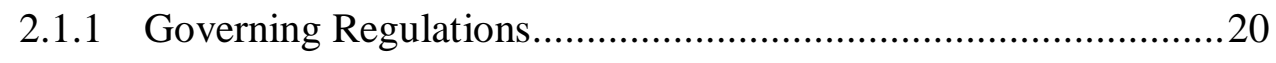

2.1.2 Clinical Trial Process …………………………....................22

2.2 Mechanical Joint Simulators (Cadaveric Knee Simulators) ......................23

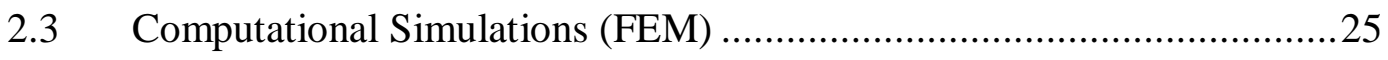


Chapter 3 Technical Background...................................................................................28

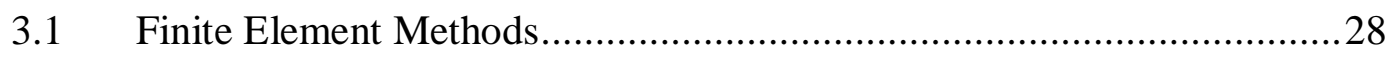

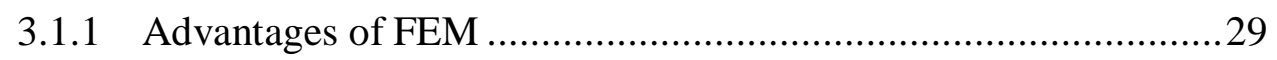

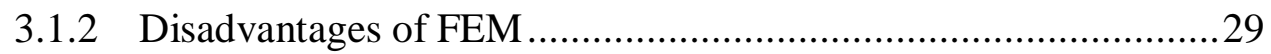

3.2 Shape Function Models ..................................................................... 30

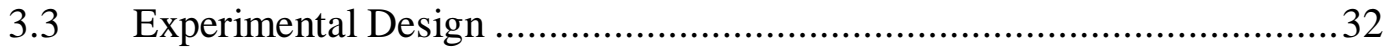

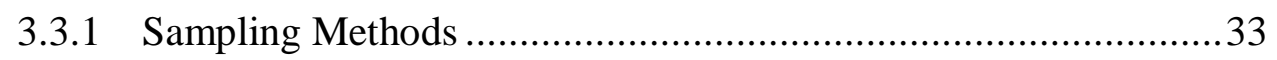

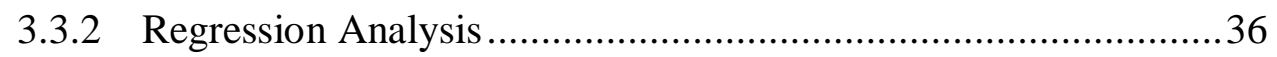

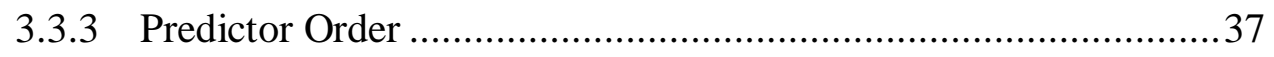

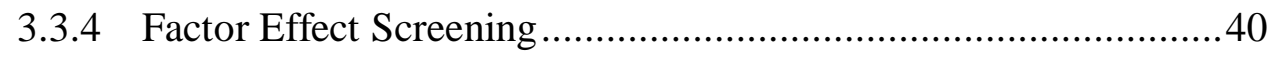

Chapter 4 Manuscript "Development of a Statistical Shape-Function Model of the Implanted Knee for Real-Time Prediction of Joint Mechanics”..41

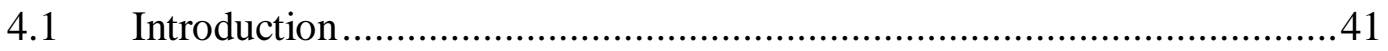

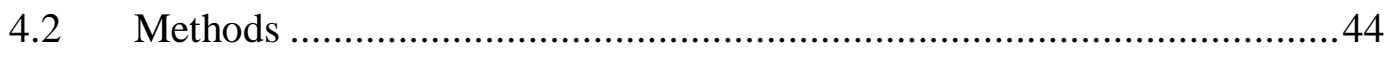

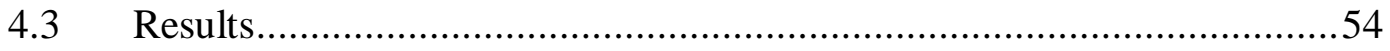

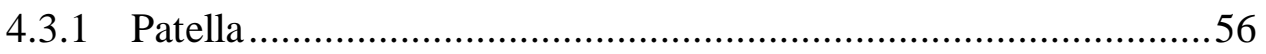

4.3.2 Ligament Elongation and Muscle Forces.......................................57

4.3.3 Tibiofemoral Kinematics..........................................................59

4.3.4 Tibiofemoral Joint Loads ……………………….......................59

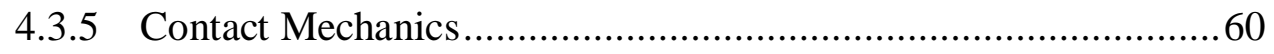

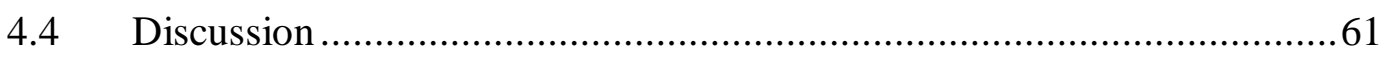

Chapter 5 Conclusions and Future Directions.............................................................65

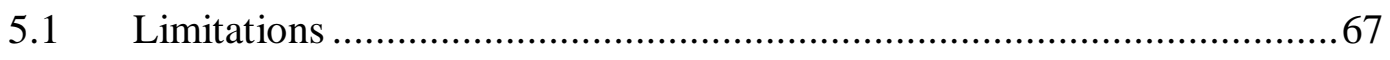

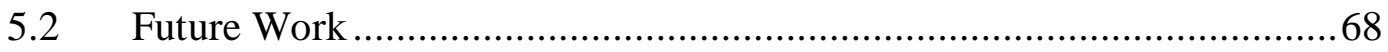


5.2.1 Shape-Function Model Improvements ................................68

5.2.2 Additional Parameterized Joints .............................................. 70

5.2.3 Graphical User Interface ................................................ 72

References ................................................................................................................................74

Appendix A: Combined Set - Two Sample T-Test Factor Sensitivities ......................A1

A.1 Tibiofemoral Contact Mechanics .................................................... A2

A.2 Tibiofemoral Joint Loads........................................................ A 8

A.3 Tibiofemoral Kinematics ........................................................ A13

A.4 Tibiofemoral Ligaments ................................................... A17

A.5 Patellofemoral Contact and Joint Mechanics, Kinematics, and Ligaments

Appendix B: Design Set - Two Sample T-Test Factor Sensitivities .......................... B1

B.1 Tibiofemoral Contact Mechanics ................................................... B4

B.2 Tibiofemoral Joint Loads..................................................... B5

B.3 Tibiofemoral Kinematics ........................................................... B6

B.4 Tibiofemoral Ligaments ................................................. 77

B.5 Patellofemoral Contact and Joint Mechanics, Kinematics, and Ligaments

Appendix C: Surgical Set - Two Sample T-Test Factor Sensitivities ........................ C1

C.1 Tibiofemoral Contact Mechanics ................................................. C3

C.2 Tibiofemoral Joint Loads ......................................................... 4

C.3 Tibiofemoral Kinematics ........................................................... C5

C.4 Tibiofemoral Ligaments ........................................................ C6 
C.5 Patellofemoral Contact and Joint Mechanics, Kinematics, and Ligaments

C7 


\section{LIST OF TABLES}

Table 1. Parameter ranges used in training and test data sets for design and surgical parameter sets. Implant geometry was parameterized using nine variables with ranges based on the geometric domain of commercially available TKA components. Six surgical alignment predictors were included, with ranges chosen to capture those of both mechanically and kinematically aligned knee replacements.

Table 2. Predicted outputs organized by functional groupings used in factor effect screening. Four groups were created for the tibiofemoral joint, while a single group collected all outputs for the patellofemoral joint.

Table 3. Average normalized RMS errors for all outputs (\%). Across all three parameter sets, doubling the sample rate while using quadratic predictors with interactions reduced errors by an average of $30.1 \%$. Further investigation of sensitivity to predictor order was performed on the design set, due to it having the largest reduction in error with increasing order. .54

Table 4. Significance by factor for the tibio-femoral contact mechanics group from the combined parameter set.

Table 5. Significance by response for the tibio-femoral contact mechanics group from the combined parameter set.

Table 6. Significance by factor for the tibio-femoral joint loads group from the combined parameter set. A9

Table 7. Significance by response for the tibio-femoral joint loads group from the combined parameter set.

Table 8. Significance by factor for the tibio-femoral kinematics group from the combined parameter set.

Table 9. Significance by response for the tibio-femoral kinematics group from the combined parameter set.

Table 10. Significance by factor for the tibio-femoral ligaments group from the combined parameter set. 
Table 11. Significance by response for the tibio-femoral ligaments group from the combined parameter set. ....................................................... 21

Table 12. Significance by factor for the patellar mechanics group from the combined parameter set.

Table 13. Significance by response for the patellar mechanics group from the combined parameter set.

Table 14. Significance of response variables for the Cam Radius parameter. This parameter was removed from the combined factor data set.

Table 15. Significance of response variables for the Femoral F-E alignment parameter. This parameter was removed from the combined factor data set. $\mathrm{C} 2$ 


\section{LIST OF FIGURES}

Figure 1. An overview of knee joint anatomy. The knee joint is the largest joint in the body, and any reduction in joint functionality will cause considerable burden to a person. Image adapted from ("Total Knee Replacement OrthoInfo - AAOS," 2019)................................................................

Figure 2. Osteoarthritis is a frequently slowly progressive joint disease typically seen in middle-aged to elderly people. In OA, the cartilage between the bones in the joint breaks down because of mechanical stress, further causing additional stress to the bones, which causes them to slowly increase in size and eventual joint failure. Image adapted from ("Total Knee Replacement - OrthoInfo - AAOS,” 2019).

Figure 3. X-ray scans showing a healthy knee (left), as well as a knee suffering from bow-leg and severe OA (right). Arrows mark the deterioration of cartilage causing a loss of spacing between the bones. Image from ("Total Knee Replacement - OrthoInfo - AAOS,” 2019).

Figure 4. An illustration depicting a knee afflicted with OA (left), and an implanted joint used in total knee arthroplasty (right). Knee replacement involves resurfacing of the bones in the joint with metal components, and a friction reducing polymer spacer called the tibial insert. Image adapted from (“Total Knee Replacement - OrthoInfo - AAOS," 2019).

Figure 5. An infographic depicting the use of FEA simulations to train a statistical shape-function model for the instantaneous prediction of joint mechanics outputs. Once the training is completed, the prediction plots will be generated using computationally inexpensive regression analysis.

Figure 6. An overview of the implant design process from initial planning to postmarket surveillance. The three main methods of investigating implant design effectiveness are prototyping, computational tools, and clinical trials. Image adapted from (Aitchison et al., 2009). 
Figure 7. The Oxford knee rig. A 6-DOF joint is made possible by allowing the hip assembly to move vertically relative to the ankle assembly, and by allowing specific rotations within the hip and ankle mechanism. The hip assembly is allowed motion within the flexion-extension and varus-valgus rotations, while the ankle assembly is allowed motion within the flexionextension, varus-valgus, and internal-external tibial clinical rotations. Image adapted from (Zavatsky, 1997).............................................24

Figure 8. A knee implant design capable of telemetric measurement through usage of four load sensors imbedded in the base of the tibial component. Image adapted from (Almouahed et al., 2017)...........................................26

Figure 9. Factorial designs only produce good models when we can collect accurate data, which was not possible in the case of this implant design (left), which used the tibial insert as a launch-ramp to dislocation (right). Other sampling methods do not require such a large number of samples and have a better spread throughout the design space, mitigating the loss of accuracy when missing a single data point.

Figure 10. An example of a full factorial (left) and central composite (right) design spaces for an experiment with three factors. A full factorial design includes every combination of factors set to high and low levels, encoded to be \pm 1 . To extend a factorial design with $k$ factors into a central composite design, we add additional "star points" at a distance $\pm 2 k / 4$ away from the mean factor value. In two and three dimensions, these additional points will circumscribe a circle or sphere with the original points.

Figure 11. Sampling a two-factor design space using a Latin square is analogous to placing rooks on a chessboard such that none of them are able to capture each other. One of the benefits of LHS is being able to select the number of samples independently from the number of factors; we can subdivide the chess board as little or as much as we want.

Figure 12. An example of the low and high factor groupings used when performing factor effect screening on a three-factor design space. The difference between average response of the high and low factor values is used for ranking the importance for each individual factor. 
Figure 13. Finite element model of knee joint TKA. Ligaments modeled in this simulation included medial and lateral collateral ligaments (MCL, LCL), anterior lateral capsule (ALC), popliteofibular ligament (PFL), posteromedial capsule (PMC), posterior capsule (PCAP), and posterior oblique structures (POL). The quadriceps muscle, which was separated into rectus femoris plus vastus intermedius (RF+VI), vastus lateralis (VL) and vastus medialis (VM) bundles, was represented by 2-D fiber-reinforced membrane elements. Patellofemoral ligaments and patellar ligament (PL) were also included.

Figure 14. Design and surgical alignment parameters, with all surgical alignments shown positive. Implant geometry (red) was parameterized using nine variables: femoral condyle distal, posterior and coronal radii, tibial insert anterior, posterior and coronal plane conformity, trochlear orientation and M-L position, and coronal plane curvature of the cam mechanism. Six surgical alignment predictors (black) included: tibial insert and femoral implant V-V and I-E alignment, femoral F-E alignment, and tibial insert slope, with ranges chosen to capture those of both mechanically and kinematically aligned knee replacements.

Figure 15. Pareto charts justifying the removal of femoral F-E alignment and cam radius parameters from the combination set, developed from factor effect screening analysis for the surgical alignment (top) and design geometry (bottom) parameters. Factor effects screening was performed by dividing output results into low and high factor blocks, where each single predictor variable was categorized as low when set below its average value, and high when set above average. For every combination of output and factor, the magnitude of the difference between the high block and the low block was taken and normalized by dividing by the average.

Figure 16. Overlayed area plots of average normalized RMS error by functional group. Within each set of parameters, highest errors are associated with linear predictors using half-sized samples, and are reduced by keeping linear predictors while doubling the sampling rate, then minimized using doubled sample rate with quadratic predictors with interaction terms. Joint loads and contact mechanics functional groups produced larger differentiation between each set, with the small sample rate with linear predictor sets showing average differences in errors of $5.6 \%$ and $5.0 \%$ when going from surgical to design, and design to combination sets, respectively. 
Figure 17. Select results from the ligament elongation functional group, from four randomly generated knees within the combination test set using a LHS sampling of 130 simulations with linear predictors. This functional group had normalized mean RMS errors, averaged by group, below 5\% across all parameter sets, with errors resulting in $1.61 \%, 3.30 \%$, and $3.97 \%$ for the surgical, design, and combined sets, respectively.

Figure 18. Prediction of kinematic results from four randomly generated knees for tibiofemoral A-P and S-I displacement, and I-E and V-V rotation of the combination parameter set using 10 simulations per parameter with linear predictors. Kinematics predictions of the TF joint resulted in normalized mean RMS errors, averaged by group, of $3.78 \%$ for the surgical, $11.3 \%$ for the design, and $10.8 \%$ for the combination parameter sets.

Figure 19. Representative contact mechanics and joint loads prediction results from the combination set, using a sample size of 130 simulations and linear predictors for regression analysis. Four randomly generated knees were selected from each functional group. From the contact mechanics group, the surgical set had a normalized mean RMS error, averaged by group, of $7.97 \%$, while the design set scored $15.3 \%$, and the combination set resulted in an error of $19.2 \%$. For the surgical set, normalized mean RMS joint load errors, averaged by group, were only $8.38 \%$, whereas the design set resulted in an error of $14.2 \%$, and the combined set had the largest error of $18.4 \%$ for this group.

Figure 20. Significance rankings for the tibiofemoral contact mechanics group of the combined set. All unique response variables are captured by the femoral $\mathrm{V}-\mathrm{V}$, tibial slope, and femoral coronal radius factors.

Figure 21. Joint loads factor rankings from the combined parameter set. All unique response variables are captured by the femoral posterior radius and V-V alignment parameters.

Figure 22. Factor significance rankings from the combined set, where the tibial slope is the most significant factor, capturing all unique response variables by itself.

Figure 23. Combined set tibiofemoral ligament group factor rankings. Interestingly, a maximum of $81 \%$ of the unique response variables are captured by the first five factors. Some of the ligaments group underwent little activity during a deep knee bend, and this will likely have contributed to the missing $19 \%$ of captured response variables. 
Figure 24. Factor significance rankings for the patellofemoral functional group of the combined set. The first four factors capture the maximum of $78 \%$ of unique response variables, which is likely due to the inclusion of ligament elongations and forces within this functional group........................A24

Figure 25. Contact mechanics factor rankings for the design set. A maximum of $90 \%$ of the unique response variables is captured by femoral posterior radius and tibial coronal conformity factors. The additional $10 \%$ were captured when moving to the combined set, showing that some of the response variables were more dependent on surgical alignment parameters......... B4

Figure 26. Design set joint load factor significance rankings. Every unique response variable is captured when including the first five factors, and tibial anterior conformity did not capture any response variables. This lack of significance is likely due to the activity under study, as the knee will roll back during a deep-knee bend.

Figure 27. Kinematics factor significance rankings for the design set. All unique response variables were captured by the femoral posterior radius factor, and none of them were significantly influenced by the cam radius........ B6

Figure 28. Factor significance rankings for the tibiofemoral ligaments functional group. Only $78 \%$ of the unique response variables were captured by the first four factors, with cam radius and femoral coronal radius proving insignificant for any of them. These results are similar to the combination factor set, though there was modest improvements when including surgical alignment parameters.........................................................

Figure 29. Patellofemoral factor significance ratings for the design set. Cam radius and femoral coronal radius again proved insignificant, but only $81 \%$ of the unique response variables were captured by the three highest ranked factors. B8

Figure 30. Contact mechanics factor significance rankings for the surgical parameter set. The femoral $\mathrm{V}-\mathrm{V}$ factor captures $80 \%$ of the unique response variables within this functional group, and the remaining $20 \%$ were not captured until introducing design parameters in the combined set.

Figure 31. Joint loads factor significance rankings for the surgical set. All unique responses were captured by the tibial I-E surgical alignment factor........ C4

Figure 32. Surgical set factor significance rankings for the tibiofemoral kinematics functional group. Every unique response within this group was captured by the tibial slope parameter. C5 
Figure 33. Tibiofemoral ligaments factor significance rankings for the surgical parameter set. A maximum of $78 \%$ of the unique response variables were captured by the first three factors. It is likely that some of the ligament response is driven by the activity under study, rather than the individual factors. C6

Figure 34. Factor significance rankings for the patellofemoral functional group of the surgical set. Only $58 \%$ of the unique response variables were captured by the first five factors, suggesting that design parameters are more significant for this functional group. 


\section{LIST OF ABBREVIATIONS}

\begin{tabular}{ll} 
A-P & Anterior-Posterior \\
ALC & Anterior Lateral Capsule \\
CDC & Centers of Disease Control and Prevention \\
CLT & Central Limit Theorem \\
DKB & Deep-Knee Bend \\
DOF & Degrees of Freedom \\
FDA & Food and Drug Administration \\
FE(M) & Finite Element (Method) \\
F-E & Flexion-Extension \\
GS & Grood-Suntay \\
GUI & Graphical User Interface \\
IDE & Lateral Collateral Ligament \\
LCH & Instividual Device Exemption \\
LCL & Internal-External \\
\hline
\end{tabular}




\begin{tabular}{|c|c|}
\hline LPOL & Lateral Posteromedial Capsule \\
\hline MCL & Medial Collateral Ligament \\
\hline MPOL & Medial Posteromedial Capsule \\
\hline M-L & Medial-Lateral \\
\hline $\mathrm{NIH}$ & National Institutes of Health \\
\hline NSAIDs & Nonsteroidal Anti-Inflammatory Drugs \\
\hline $\mathrm{OA}$ & Osteoarthritis \\
\hline $\mathrm{PF}$ & Patellofemoral \\
\hline PFL & Popliteofibular Ligament -or- Patellofemoral Ligament \\
\hline PI & Proportional-Integrative \\
\hline PL & Patellar Ligament \\
\hline PMA & Premarket Authorization \\
\hline PMC & Posteromedial Capsule \\
\hline PS & Posterior Stabilized \\
\hline$Q+I$ & Quadratic plus Interactions \\
\hline $\mathrm{RF}$ & Rectus Femorus \\
\hline RMS(E) & Root-Mean-Square (Error) \\
\hline SFM & Shape-Function Model \\
\hline S-I & Superior-Inferior \\
\hline
\end{tabular}


TF

TKA

VI

VL

VM

V-V

2-D

$+\mathrm{VE}$
Tibiofemoral

Total Knee Arthroplasty

Vastus Intermedius

Vastus Lateralis

Vastus Medialis

Varus-Valgus

Two-Dimensional

Positive 


\section{CHAPTER 1}

\section{INTRODUCTION}

The American population is getting older, on average, and this is placing strain on our in-

place healthcare systems. There is a shortage of primary care physicians, with $13 \%$ of Americans (44 million) residing within a county with inadequate coverage of primary care providers, defined as less than one primary care physician per 2,000 people (“Addressing the Nation's Primary Care Shortage: Advanced Practice Clinicians and Innovative Care Delivery Models,” 2018). By the year 2030, the U.S. population is expected to increase by 27 million people, equivalent to $8 \%$, but the number of people aged 65 and older will increase by $38 \%$ (12 million people). Of those people aged 65 and older, the number of people living with at least one chronic disease will increase by over 33\%, from 43 million to 59 million. One of the most common diseases affecting older adults is arthritis, with an estimated 54.4 million U.S. adults $(22.7 \%)$ being diagnosed between the years of 20132015 (Barbour et al., 2017). Arthritis is a blanket term for joint disease, and when coupled with the fact that knee arthroplasty is the most common surgical joint procedure within the United States (McDermott et al., 2017), affecting some 752,941 Americans in 2014, it becomes clear that arthritis of the knee places considerable strain on the American healthcare system. To understand the challenges facing the current healthcare system, and the research 


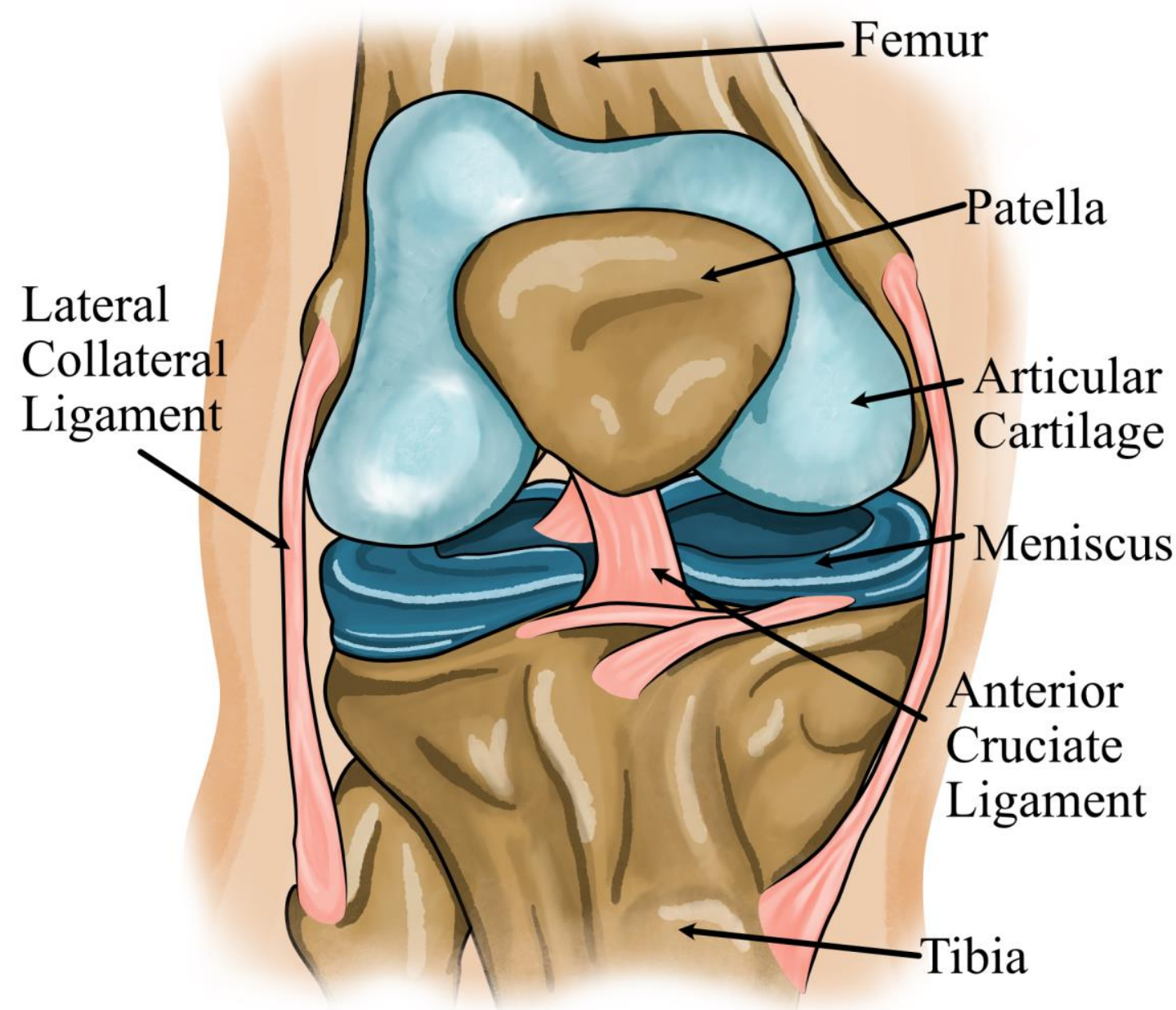

Figure 1. An overview of knee joint anatomy. The knee joint is the largest joint in the body, and any reduction in joint functionality will cause considerable burden to a person. Image adapted from ("Total Knee Replacement - OrthoInfo - AAOS," 2019).

being performed to combat them, we must begin by understanding the knee, and the diseases which affect it.

\subsection{The Knee}

The knee is one of the most complex joints in the body and having healthy knees is required to perform most everyday activities. The knee joint is made up of the distal end 
of the femur, the proximal end of the tibia, and the patella (Figure 1). The contact surfaces of these three bones are protected by articular cartilage: a smooth, low-friction material that protects the bones and facilitates easy articulation. The menisci, C-shaped, shock absorbing wedges are located between the femur and tibia, and act to cushion the joint. Joint stability is provided by large ligaments which hold the femur and tibia together, while the long quadriceps muscles give the joint its strength. The rest of the joint surfaces are covered by a thin lining which releases a fluid that lubricates the cartilage called the synovial membrane. This fluid filled membrane acts to reduce friction within the joint to nearly zero within the healthy knee (Basalo et al., 2007; Qian et al., 2006). All of these components work in harmony within the healthy knee, but disease or injury can disrupt their normal function, resulting in muscle weakness, reduced function, and chronic pain.

Arthritis is the most common cause of chronic knee pain and disability (Lawrence et al., 2008). There are many types of arthritis, but the types most associated with knee pain are osteoarthritis, rheumatoid arthritis, and post-traumatic arthritis.

- Osteoarthritis: This is an age-related degenerative type of arthritis caused by normal 'wear and tear'. It typically occurs in people above the age of 50, but is increasingly occurring in younger people, also. When a patient has symptoms of osteoarthritis, the cartilage that protects the bones of the knee softens and wears away. This causes the bones to rub against each other, causing stiffness and knee pain.

- Rheumatoid arthritis: The most common form of a group of disorders called inflammatory arthritis. When the synovial membrane that surrounds the joint becomes inflamed and thickened, we term this rheumatoid arthritis. Eventually, this chronic inflammation can damage the cartilage and cause cartilage loss, pain, and stiffness. 

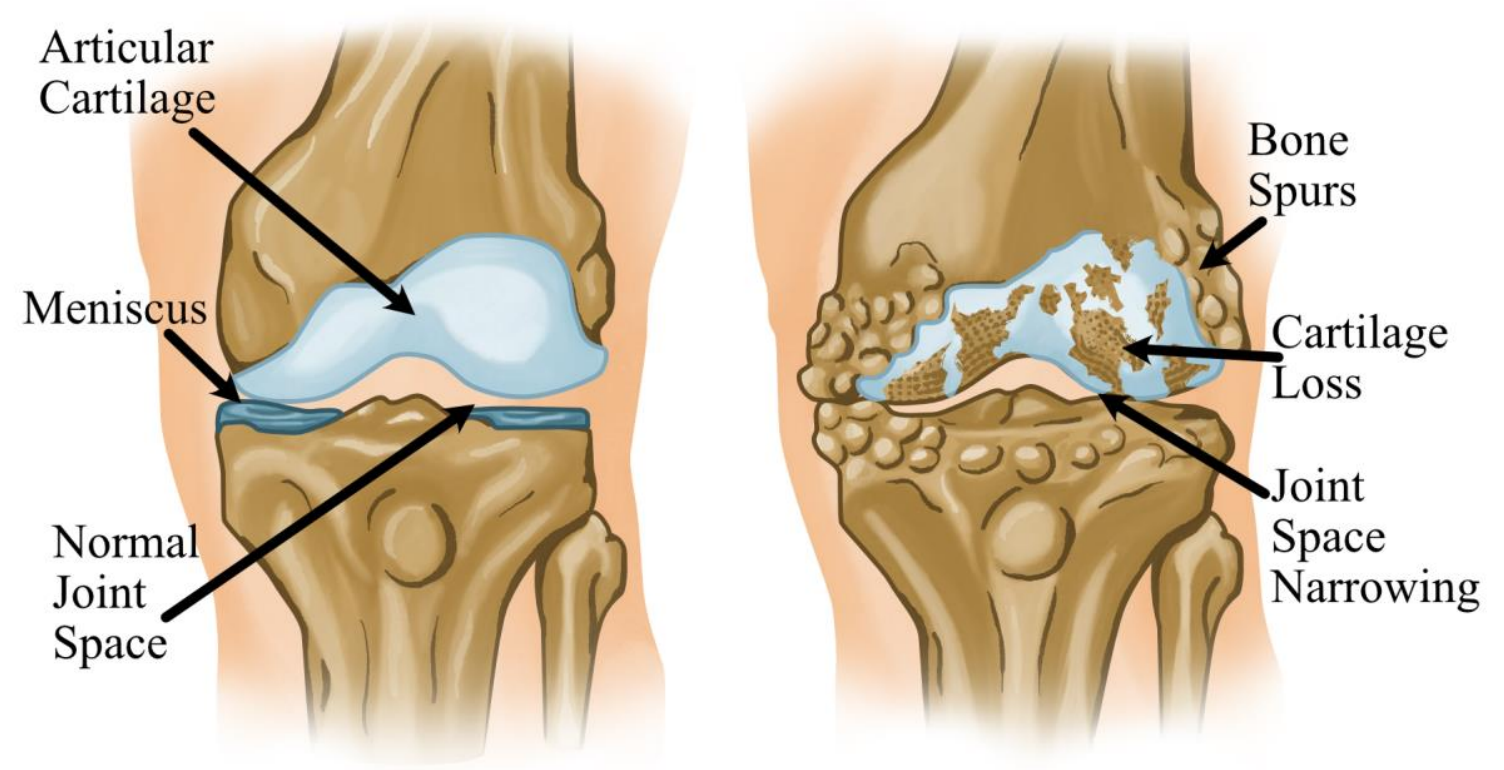

Figure 2. Osteoarthritis is a frequently slowly progressive joint disease typically seen in middle-aged to elderly people. In OA, the cartilage between the bones in the joint breaks down because of mechanical stress, further causing additional stress to the bones, which causes them to slowly increase in size and eventual joint failure. Image adapted from ("Total Knee Replacement - OrthoInfo - AAOS," 2019).

- Post-traumatic arthritis: Following a serious knee injury, tears of the knee ligaments or fractures of the bones surrounding the knee may damage the articular cartilage, causing knee pain and limiting knee function.

All of the above examples share the traits of cartilage loss, have similar symptoms, and slowly worsen with time. As osteoarthritis is the most common form of arthritis (Heidari, 2011; Lawrence et al., 2008), the following section will look at OA in detail.

\subsection{Osteoarthritis}

Osteoarthritis (OA) is a common joint disease that most often affects middle-age to elderly people. This disease affects the entire joint, involving cartilage, the joint lining, ligaments, and bone. When a patient presents with OA, it is characterized by a deterioration 
of the cartilage, tendons and ligaments, and by bony changes of the joint, which are accompanied by inflammation of the joint lining to various degrees (Lane et al., 2011) (Figure $2)$.

The patient reported symptoms of OA include:

- Joint pain and stiffness, possibly during rest as well as activity

- Knobby (lumpy) swelling at the joint

- Cracking or grinding noise with joint movement, especially when using stairs

- Decreased function and mobility of the joint

This arthritis is not limited to the knee, and also tends to occur within the hand, spine, hips, and great toe joints. According to the Johnston County Osteoarthritis Project (Murphy et al., 2008), a long-term study from the University of North Carolina and sponsored by the Centers of Disease Control and Prevention (CDC) and National Institutes of Health (NIH), the lifetime risk of developing OA of the knee is about $46 \%$, and the lifetime risk of developing OA of the hip is $25 \%$. The incidence of knee OA is rising commensurately with population age, and it is one of the leading causes of disability in older people.

\subsubsection{Patients}

OA affects people of all races and sexes, though it is much more likely to have multiple large joint involvement in African Americans (Jordan, 2015). Most often, it occurs 
in patients age 50 and older, but it can occur earlier if a person has other OA risk factors, which, in addition to older age, include:

- Obesity

- Having family members with a history of OA

- Previous traumatic joint injury or repetitive use of joints

- Joint deformity such as unequal leg length, bowlegs, or knocked knees

There is no known cure for the disease, but current treatments exist which aim to reduce pain and improve function, and most importantly, to slow disease progression.

\subsubsection{Treatments}

Most often, doctors diagnose OA based on the typical symptoms and results of a physical exam. In some cases, imaging tests such as X-ray may be useful to help rule out other joint problems or to detect the extent of disease progression (Figure 3). However, changes to the soft tissues in the joint cannot be visualized in this way, and X-ray imaging detection has proven most successful only at late stages of the disease (Lane et al., 2011). While there exists no proven treatment that can reduce the joint damage caused by OA, treatments exist which enable reduced pain and may improve affected joint function. Most often, treatment will entail a mixture of physical measures and drug therapy and, as a final resort, surgery. 

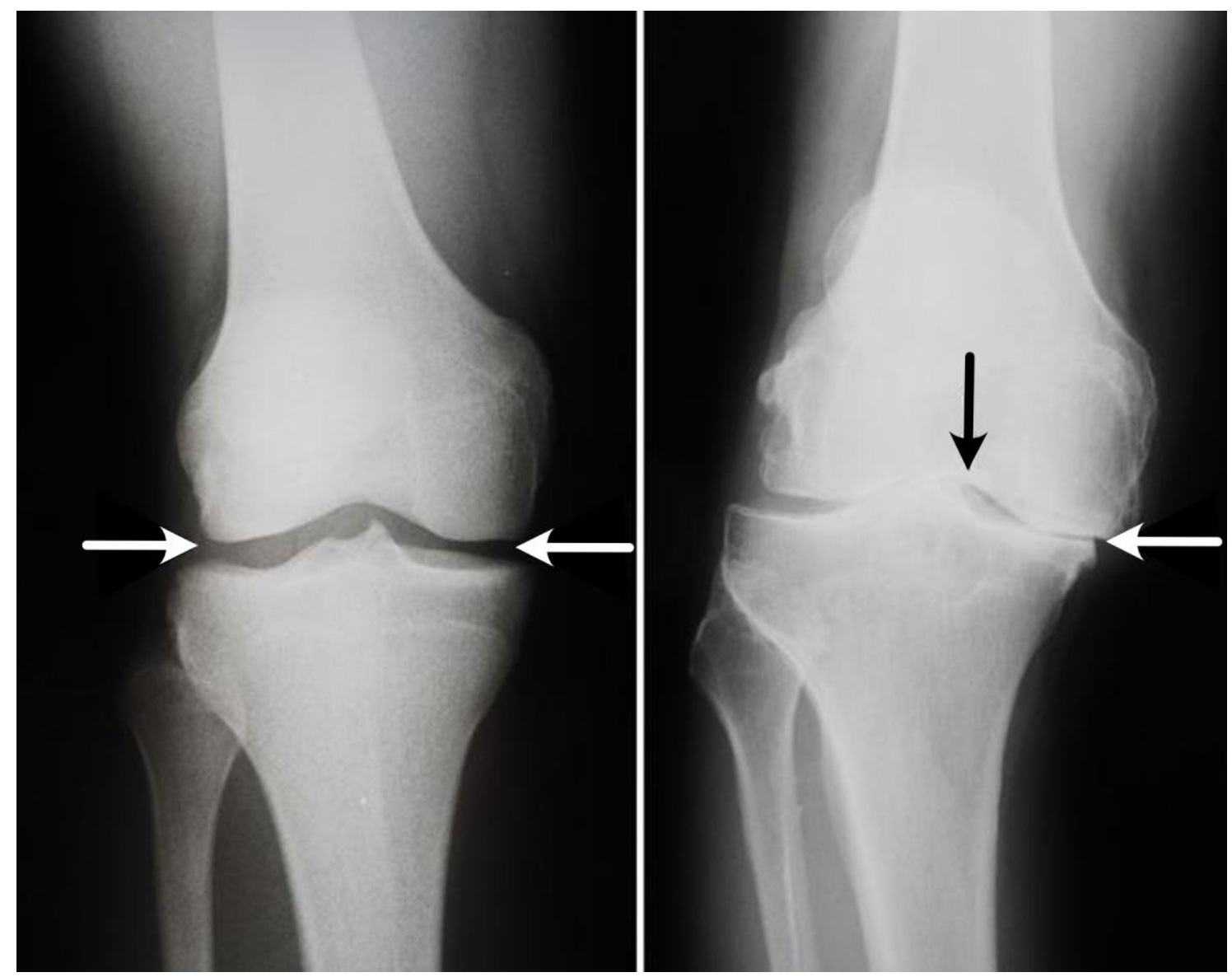

Figure 3. X-ray scans showing a healthy knee (left), as well as a knee suffering from bow-leg and severe OA (right). Arrows mark the deterioration of cartilage causing a loss of spacing between the bones. Image from ("Total Knee Replacement - OrthoInfo - AAOS," 2019).

\section{$\underline{\text { Physical Measures }}$}

Weight loss and exercise are important in the treatment OA. Excess weight puts stress on a patient's knee joints, hips, and lower back. For every 10 pounds of weight lost over 10 years, the chance of developing knee OA is reduced by up to $50 \%$ ("NIH Consensus Statement on Total Knee Replacement,” 2003). Exercise can improve muscle strength, decrease joint pain and stiffness, and lower the chance of disability caused by OA. If the disease has not progressed very far, assistive support devices, such as orthotics or a walking 
cane, can help patients to continue performing daily activities. For short term relief of OA symptoms, heat or cold therapy may help (Brosseau et al., 2003).

\section{Drug Therapy}

Forms of drug therapy used in the treatment of OA include topical, oral, and injections. You apply topical drugs such as capsaicin cream, lidocaine and diclofenac gel directly to the skin of the affected joints. Frequently, oral pain relievers such as acetaminophen and nonsteroidal anti-inflammatory drugs (NSAIDs), which decrease swelling and pain are common first treatments. In 2010, the Food and Drug Administration (FDA) approved the use of duloxetine for chronic musculoskeletal pain, which allows its use in treating OA. If the disease has progressed to a moderate point, patients may be prescribed stronger medications, such as narcotics. For providing moderate length relief (months) from $\mathrm{OA}$, joint injections with corticosteroids or hyaluronic acid may be given. When the hyaluronic acid lubricant is used across multiple treatments, it may help delay the need for a knee replacement by up to a few years in some cases (Askari et al., 2016). 

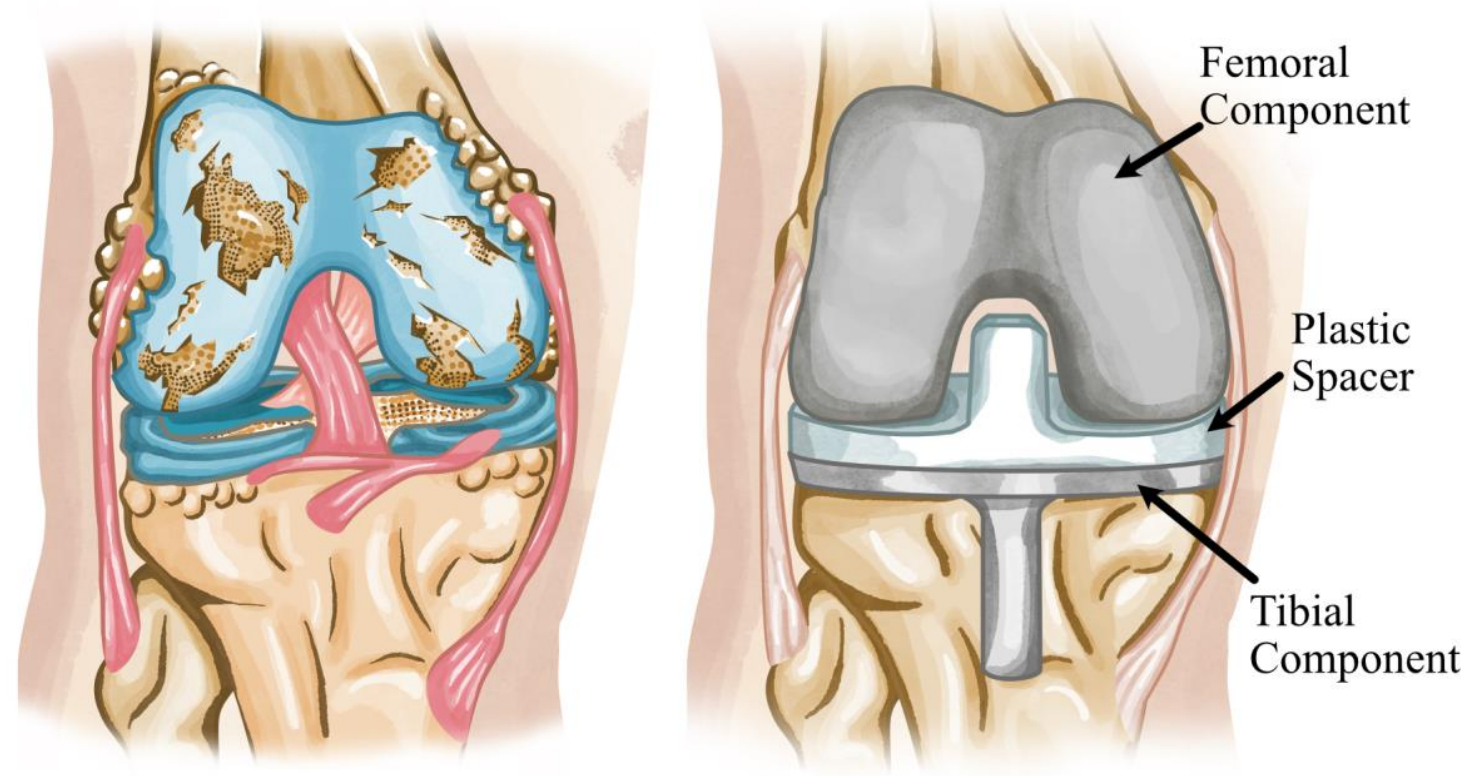

Figure 4. An illustration depicting a knee afflicted with OA (left), and an implanted joint used in total knee arthroplasty (right). Knee replacement involves resurfacing of the bones in the joint with metal components, and a friction reducing polymer spacer called the tibial insert. Image adapted from ("Total Knee Replacement - OrthoInfo - AAOS," 2019).

\section{Surgery}

For severe cases of OA, where the joint is seriously damaged, or when other treatments have failed to relieve pain, or the patient has a major loss of function, surgical treatment becomes an option. Surgery may involve repair of the joint done through small incisions, known as arthroscopy (Barnes et al., 2006; Law et al., 2019). If the joint damage cannot be repaired in this way, joint replacement is the next option (Figure 4). Joint replacement may be recommended when:

- Severe knee pain or stiffness limits the patient's everyday activities, including walking, climbing stairs, and getting in and out of chairs. Candidates for knee replacement may find it hard to walk more than a few blocks without significant pain and they may already be using a cane or walker 
- Patients experience moderate or severe knee pain while resting, whether this occurs during the day or at night

- Chronic knee inflammation and swelling which has not shown improvement with rest or the administration of medications

- A patient exhibits a Knee deformity known to cause rapid OA progression, such as a bowing in or out of the knee joint

- Failure to show substantial improvement with other forms of treatment such as anti-inflammatory medications, cortisone and lubricating injections, physical therapy, or arthroscopic surgeries

The modern version of knee replacement surgery was first performed by Frank Gunston in 1968 (Shetty et al., 2003), and improvements in surgical materials and techniques over the past 50 years have greatly increased its safety and effectiveness. Today, total knee replacements are one of the most frequent and successful procedures in the United States. As of 2014, combined partial and total knee replacement is ranked the third most frequent operating room procedures, with more than 700,000 knee replacements performed each year in the United States (McDermott et al., 2017).

When performing a total knee replacement surgery, the procedure may be divided into four primary steps:

1. Bone preparation: A small layer of bone, including the damaged cartilage surfaces at the end of the femur and tibia are removed 
2. Resurfacing the tibio-femoral joint: The removed cartilage and bone is replaced with metal that has been shaped in order to recreate the smooth surface of the joint. These metal parts may be cemented or "press-fit" into the bone, and some materials exist which can chemically bond with existing bone

3. Resurfacing the patella: If wear is extensive, the contacting surface of the patella is resurfaced with plastic

4. Tibial spacer insertion: To prevent metal-on-metal wear, and to reduce the friction within the joint, a plastic spacer is inserted between the metal components

For $90 \%$ of people who have total knee replacement surgery, there is a reliable reduction of knee pain and a significant improvement in the ability to perform common activities of daily living, with full restoration of pre-disease joint function being the upper limit of surgical outcomes (Bade et al., 2010; "NIH Consensus Statement on Total Knee Replacement," 2003). Most patients can expect to be able to almost fully straighten the replaced knee and to bend the knee sufficiently for daily tasks, but kneeling is sometimes uncomfortable, although not harmful. Patients may also feel some stiffness, particularly when performing activities with excessive bending. Most people also feel or hear some clicking of the metal and plastic when using their joint. These symptoms often reduce with time, and most patients find them to be a tolerable improvement over pre-surgery function.

Similar to our natural knee, over time with normal use and activity, every knee replacement implant begins to exhibit wear within the tibial insert. Excessive activity or weight may contribute to the rate of wear, possibly causing the knee replacement to loosen and patients may begin to once again experience pain. Surgeons advise most patients to 
avoid high-impact activities for the rest of their post-surgery lives. Low-impact activities which a patient can expect to safely engage in following total knee replacement include walking, swimming, golf, driving, light hiking, biking, ballroom dancing, and other lowimpact sports (Swanson et al., 2009).

Rehabilitation following total knee replacement surgery typically begins the day after surgery and, for some cases, patients may begin moving their knee on the same day as receiving surgery. Following surgery, surgeons will typically instruct patients to do the following:

- Participate in regular light exercise programs to maintain proper strength and mobility of their new knee.

- Take precautions to avoid falls and injuries

- Return for periodic follow-up appointments with the orthopaedic surgeon, generally once per year

When patients stick to this type of low-impact activity and follow their surgeon's guidelines, they can expect their knee replacement to last for many years. Today, over $90 \%$ of modern total knee replacements are still functioning correctly 15 years following surgery (Abdel et al., 2011).

In general, full restoration of joint motion is uncommon, with functional performance in patients one year following TKA being reported with an $18 \%$ slower walking speed, 51\% slower stair climbing speed, and a reduction in quadriceps strength of nearly $40 \%$ (Bade et al., 2010). While patient outcomes following TKA are already very good, up to $20 \%$ of knee replacement patients are dissatisfied with the outcome of their knee surgery (Halawi et al., 2019), and, when coupled with the fact that 700,000 knee replacements are 

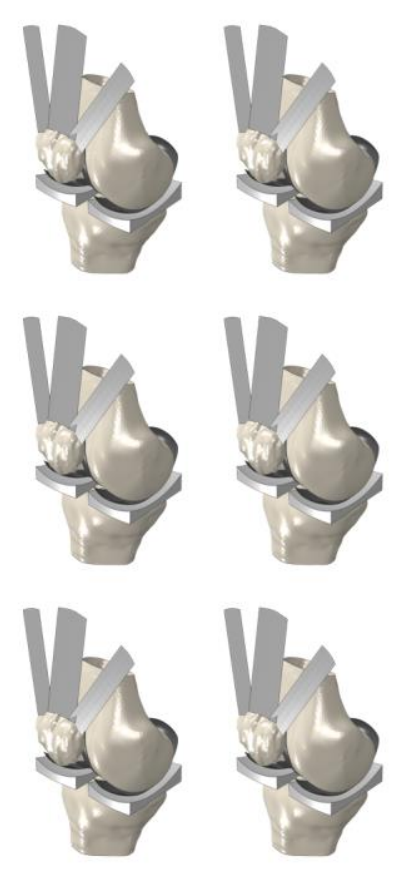
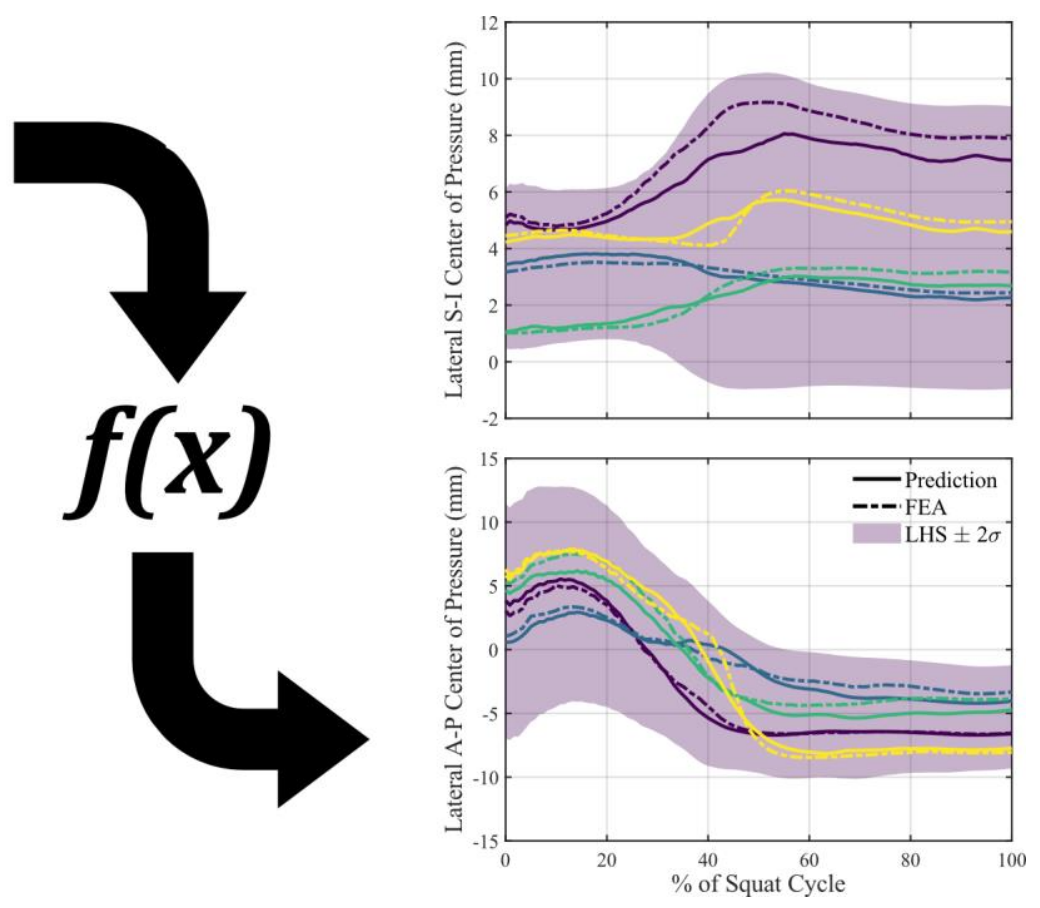

Figure 5. An infographic depicting the use of FEA simulations to train a statistical shape-function model for the instantaneous prediction of joint mechanics outputs. Once the training is completed, the prediction plots will be generated using computationally inexpensive regression analysis.

performed annually within the United States, this works out to up to 140,000 dissatisfied patients. Improvements to implant design and surgical technique are active areas of research, with the goal of improving the current techniques until patient functionality can be completely restored to pre-surgery levels. The present thesis work contributes work towards this goal with the aim of reducing the time and expertise requirements associated with implant design through the use of finite element simulations as a training set in developing a novel computational joint mechanics prediction model (Figure 5).

\subsection{Thesis Statement}

Computational tools such as finite element analysis (FEA) are already in use within the medical device industry (Aitchison et al., 2009), and aid in reducing the time-to-market 
when developing a new implant design. However, the use of FEA still predicates considerable investments of time, capital, and expertise. The present thesis research aims to provide a computational framework which will allow for the instantaneous prediction of implanted knee joint mechanics in a manner that reduces the requisite knowledge and expertise when compared to the development of an FEA model. The specific objectives are as follows:

- Develop a set of parameterized FEA simulations of the implanted knee performing a deep knee bend (DKB) which may be used to train a statistical prediction model. Model parameters will include geometric factors and surgical alignments

- Determine sensitivity of key joint mechanics outputs, such as joint loads, contact mechanics, and kinematics of the tibiofemoral and patellofemoral joints, and ligament elongations and muscle forces, to changes in individual model parameters

- Produce a statistical model which enables the real-time prediction of the previously mentioned joint mechanics groups

- Validation of the statistical models against an additional test set of FEA simulations by quantifying the resulting errors for every joint mechanic output

The present thesis is organized in the following way:

Chapter 2 summarizes current methods used within the total knee replacement design research community. First, an overview of the implant design process is provided, where it is shown that clinical trials, mechanical joint simulators, and computational tools are the three primary ways researchers and manufacturers study knee implant design. For 
each method, the process required, and the advantages and disadvantages of the technique are summarized.

Chapter 3 outlines the methods used in the statistical modeling of joint mechanics present within this study. It begins with an explanation of statistical shape functions, then continues to detail the components of experimental design including a survey of experimental sampling methods, a description of regression analysis and the nuances of the equations used in linear regression, as well as a description of one sensitivity analysis technique: factor effects screening.

Chapter 4 includes a faithful reproduction of the authors' manuscript, previously published in the Journal of Biomechanics. This manuscript provides a detailed summary of the development of the statistical prediction models which allow us to instantaneously predict joint mechanics outcomes before running a finite element simulation, as well as quantification of results and validations.

Chapter 5 gives concluding remarks and suggests future work for improving the statistical models, outlines some of the efforts made to increase the likelihood that this framework may be transferred to other models or joints, and makes suggestions towards incorporating this framework as part of a graphical user interface program to assist in implant optimization. 


\subsection{Works Published}

The following works were published during the course of this study:

Gibbons, K.D., Clary, C.W., Rullkoetter, P.J., Fitzpatrick, C.K., 2019. Development of a statistical shape-function model of the implanted knee for real-time prediction of joint mechanics. Journal of Biomechanics 88, 55-63.

https://doi.org/10.1016/j.jbiomech.2019.03.010

Gibbons, K. D., Clary, C.W., Rulkoetter, P.J., Fitzpatrick, C.K., 2019. A statistical shapefunction model of the implanted knee to predict joint mechanics. $65^{\text {th }}$ Annual Meeting of the Orthopaedic Research Society, Austin, TX, February 2019. Podium talk. 


\section{CHAPTER 2}

\section{LITERATURE REVIEW}

To understand the current research methodology surrounding surgical implants, it is important to first understand the implant design process. The implant design process is outlined using a series of seven steps (Figure 6):

1. Design feasibility studies

a. Planning stage where design inputs, commercial aspects, regulatory requirements, and design requirements are fleshed out

2. Design production

a. A narrowing of initial conceptual designs until a subset of one or more detailed designs are developed

3. Design verification

a. Using the most resource effective tools available to verify that the detailed design should operate as expected, satisfying the design requirements developed during the design feasibility stage

b. Often makes use of computational models, such as finite element methods

c. May include rapid prototyping and mechanical testing 


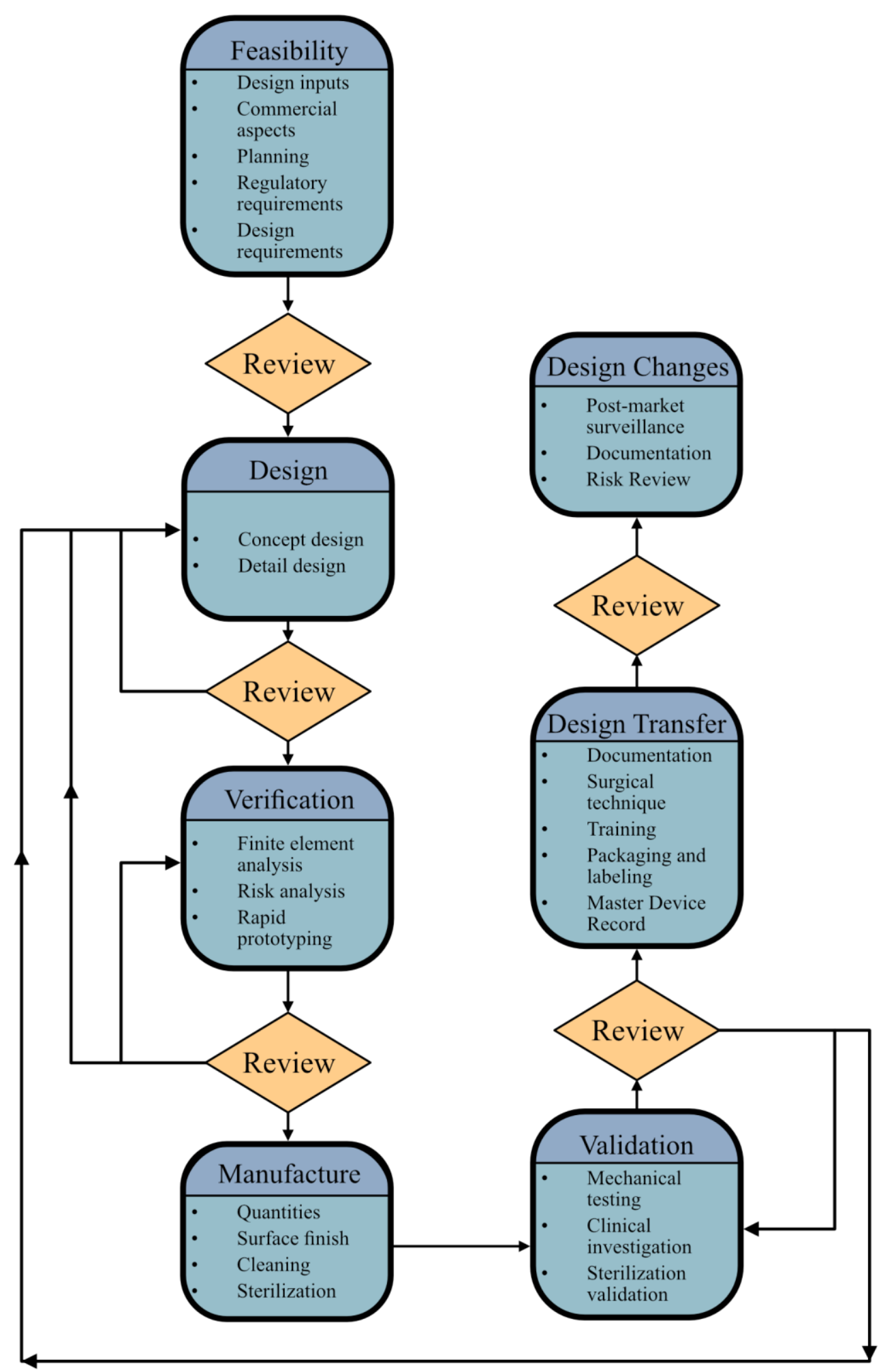

Figure 6. An overview of the implant design process from initial planning to postmarket surveillance. The three main methods of investigating implant design effectiveness are prototyping, computational tools, and clinical trials. Image adapted from (Aitchison et al., 2009). 


\section{Design manufacture}

a. Decisions must be made about the manufacturing methods used to produce the final products, where factors relating to this include the number to be produced, the surface finishes required, post machining cleaning processes, and any required sterilization processes

5. Design validation

a. Late stage prototypes will undergo more stringent mechanical testing, and possibly clinical testing as required by regulatory oversight. Sterilization processes will be tested and validated

6. Design transfer

a. Documentation and necessary clinical training protocols must be produced, packaging and labeling requirements must be implemented, and master device records must be finalized

7. Design changes (post-market)

a. Post market surveillance process must be implemented to ensure the safety of patients and healthcare workers, and feedback from these users will likely lead to new design changes that must be documented, investigated, and implemented

These phases of implant design are reviewed between each step to assess the quality of the design. It is very common to return to an earlier stage in the process for redesign, and the entire process takes an average of 3 to 7 years for an entirely new design to come to market (Van Norman, 2016). Research into implant design with the goal of improving patient outcomes and reducing time-to-market is ongoing, and the tools utilized by investigators mimic those used to design the implants themselves: clinical trials, mechanical and cadaverous testing using joint simulators, and computational studies such as FEA. 


\subsection{Clinical Trials}

Clinical trials are costly, and take a long time to perform, but generate the most comprehensive data to measure in vivo device performance. They exhibit the most regulatory oversight when studying implants, and require the approval of an institutional review board (IRB), which is a group formally designated to protect the rights, safety, and wellbeing of humans involved in the clinical trial by reviewing all aspects of the proposed study before approving its undertaking.

\section{$\underline{2.1 .1}$ Governing Regulations}

Within the United States, this regulatory oversight is handled by the Food and Drug Administration (FDA), which was initially given jurisdiction over implant devices by the 1976 Medical Device Amendments (Rogers, 1976). This amendment was later followed by the Medical Device User Fee and Modernization Act of 2002 (Greenwood, 2002), which granted the FDA the authority to collect user fees for select medical device premarket submissions and established new regulatory requirements for 'reprocessed' devices. A unique device identification system for medical devices was introduced in the 2007 FDA Amendments Act (Dingell, 2007), and over the next few years it became apparent that the present regulatory systems were becoming increasingly strained by the growing number and complexity of medical devices.

To streamline the regulatory approval process, the 2012 FDA Administration and Safety Innovation Act created the simplified De Novo pathway (Harkin, 2012), which simplified the path to approval for novel, low-to-moderate risk devices. Continuing along this trajectory, the $21^{\text {st }}$ Century Cures Act of 2016 implemented the FDA's expedited review 
program for breakthrough devices (Bonamici, 2016), permitted the use of central IRB oversite instead of local IRBs for implanted devices, and streamlined the process for exempting devices from the premarket notification requirements. The most recent legislation concerning implant design was the 2017 FDA Reauthorization Act (Walden, 2017), which included improvements to premarket review times and the National Evaluation System for health Technology and patient input. With some knowledge of the regulatory laws in place, we may now take a look at the clinical trial process, as it pertains to implanted medical devices.

The FDA assigns medical devices to one of three regulatory classes based on their intended use, whether the device is invasive or implantable, and the risks posed by the device to their users. These device classes determine the level of evidence and evaluation required to demonstrate their safety and effectiveness, and implant devices typically fall under Class III, requiring the most stringent requirements before being brought to market. A novel device will require premarket authorization (PMA), which requires proof of safety and effectiveness through the submission of clinical trials. If the device poses low-to-moderate risk, the manufacturer can apply for reclassification to Class II or I through the de novo process, which will relax the requirements to that of the $510(\mathrm{k})$ process, which requires device makers to prove that the new device is substantially equivalent to a legally marketed predicate device. A PMA, when required of device manufacturers for new designs, will necessitate the use of an Investigational Device Exemption (IDE) and review from an IRB before, during, and after a clinical trial has taken place. 


\subsubsection{Clinical Trial Process}

The first step in performing a clinical trial for an implant device is submission of a completed IDE application for review by the FDA. Following IDE approval, investigators must submit their investigational plan and report of prior investigations to the IRB at each institution where the investigation is to be conducted for review and approval. An IRB is an appropriately constituted group that has been formally designated to review and monitor biomedical research involving human subjects, and has the authority to approve, require modifications in, or disapprove research. They must be registered with the FDA, and must comply with all applicable requirements of the IRB and IDE regulations. Their purpose is to ensure that appropriate steps are taken to protect the rights, safety, and welfare of humans participating as subjects in research. Following initial IRB approval, the following requirements must be met in order to conduct the investigation in compliance with the IDE regulations:

- Labeling - The device must be labeled in accordance with the labeling provisions of the IDE regulations and must bear the statement "CAUTION Investigational Device. Limited by Federal (or United States) law to investigational use."

- Distribution - Investigational devices may only be distributed to qualified investigators

- Informed Consent - Each subject must be provided with and sign an informed consent form before being enrolled in the study

- Monitoring - All investigations must be properly monitored to protect the human subjects and assure compliance with device monitoring protocols 
- Prohibitions - Commercialization, promotion, and misrepresentation of an investigational device and prolongation of the study are prohibited

- Records and Reports - Sponsors and investigators are required to maintain specific records and make reports to investigators, IRBS, and the FDA

\subsection{Mechanical Joint Simulators (Cadaveric Knee Simulators)}

A joint simulator is a mechanical device which allows us to study manufactured joint implants within the laboratory, and are designed to mimic the in vivo characteristics of the joint, to varying degrees of success. They are a valuable tool in the pre-clinical evaluation of joint replacement devices, and a number of mechanical knee simulators, which range from standard mechanical testing machines used to measure the geometric constraint of implants under anterior-posterior (A-P) and internal-external (I-E) loads (Haider and Walker, 2005), to machines specifically designed for loading and measurement of knee joint mechanics during dynamic conditions, have been developed to perform comparative evaluation of the mechanics and wear of current or prospective knee replacement designs. The models which have been designed for dynamic conditions include the Oxford-rig type simulators (Figure 7), which apply loads to the hip, ankle, and quadriceps. This type of simulator allows for a six-degree-of-freedom (6-DOF) knee joint (Colwell et al., 2011; Maletsky and Hillberry, 2005; Varadarajan et al., 2009), and the Stanmore knee simulator which has been used to assess wear in the tibiofemoral (TF) joint (DesJardins et al., 2000). 


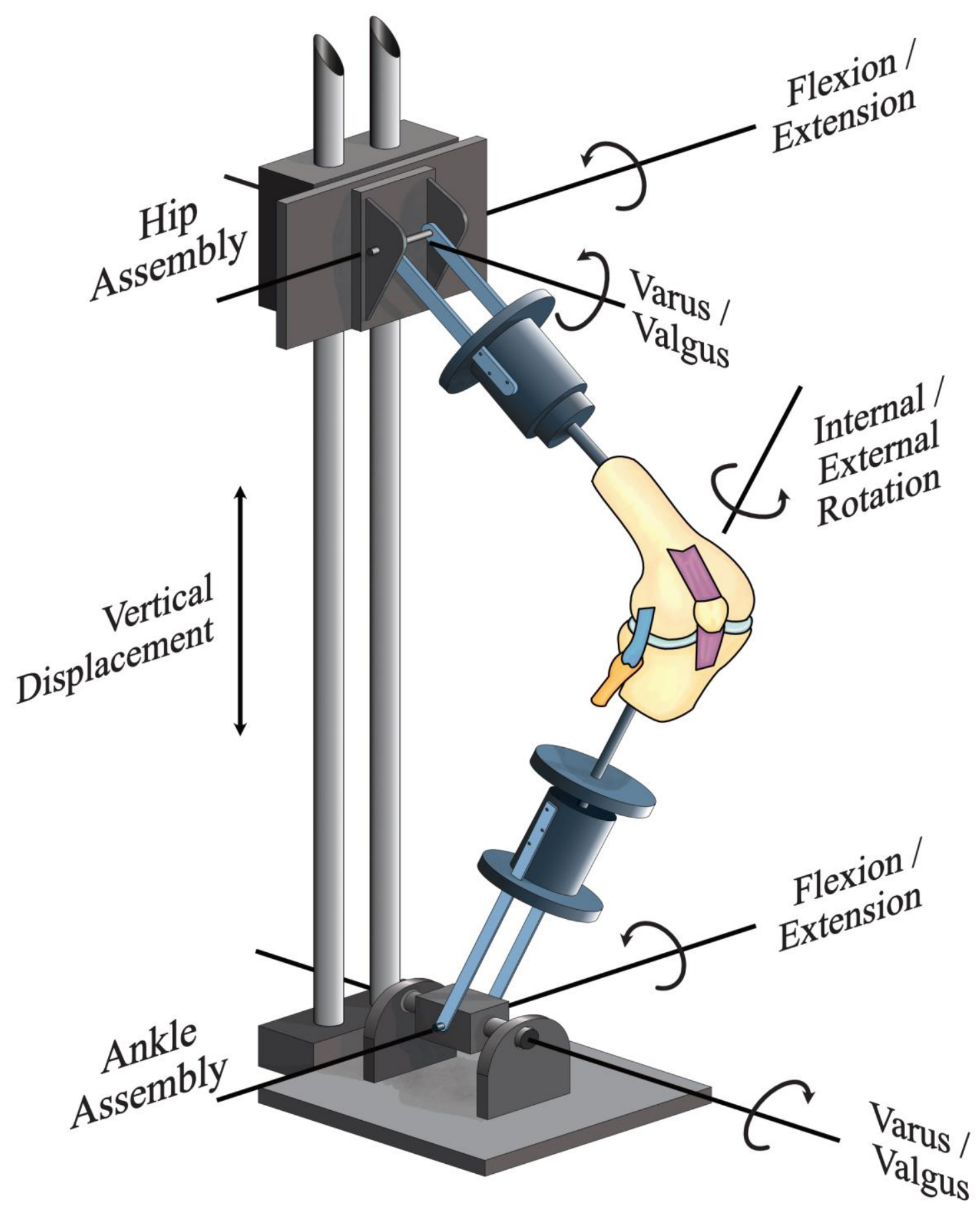

Figure 7. The Oxford knee rig. A 6-DOF joint is made possible by allowing the hip assembly to move vertically relative to the ankle assembly, and by allowing specific rotations within the hip and ankle mechanism. The hip assembly is allowed motion within the flexion-extension and varus-valgus rotations, while the ankle assembly is allowed motion within the flexion-extension, varus-valgus, and internal-external tibial clinical rotations. Image adapted from (Zavatsky, 1997). 
More recently, a new 6-DOF joint simulator has become available which facilitates testing of implants under more realistic joint conditions. This new simulator, named the VIVO simulator (AMTI, Watertown, MA), is entirely servo-controlled. When operating the VIVO, loads or motions can be applied in any combination for all six DOFs. The VIVO accepts load or kinematic profiles within the Grood and Suntay (GS) (Grood and Suntay, 1983) coordinate frame, with target profiles produced using a control system, in combination with a load sensor under the tibial component. When the user submits a loading or motion profile, they are used to calculate the desired loads or motions required by the machine's actuators, and the control system engages the signals to replicate the desired motions and loads.

Limitations associated with joint simulators include the cost and person-power required to design and fabricate the physical components, run the experimental tests, and the simulator up-time necessary for running the experiments, which can encompass weeks or months in the case of long-running wear and fatigue testing. These limitations impose constraints to the number of designs which can be tested in a reasonable time-frame, and the number of loading conditions selected for evaluation.

\subsection{Computational Simulations (FEM)}

Implant designs almost exclusively contain complicated geometry, and necessitate the modeling of biological processes and constructs. Due to these requirements, finite element methods (FEM) are a frequently used tool within this field of research. Typically, there are a lot of analogs between the design of mechanical joint simulators and the FEM 


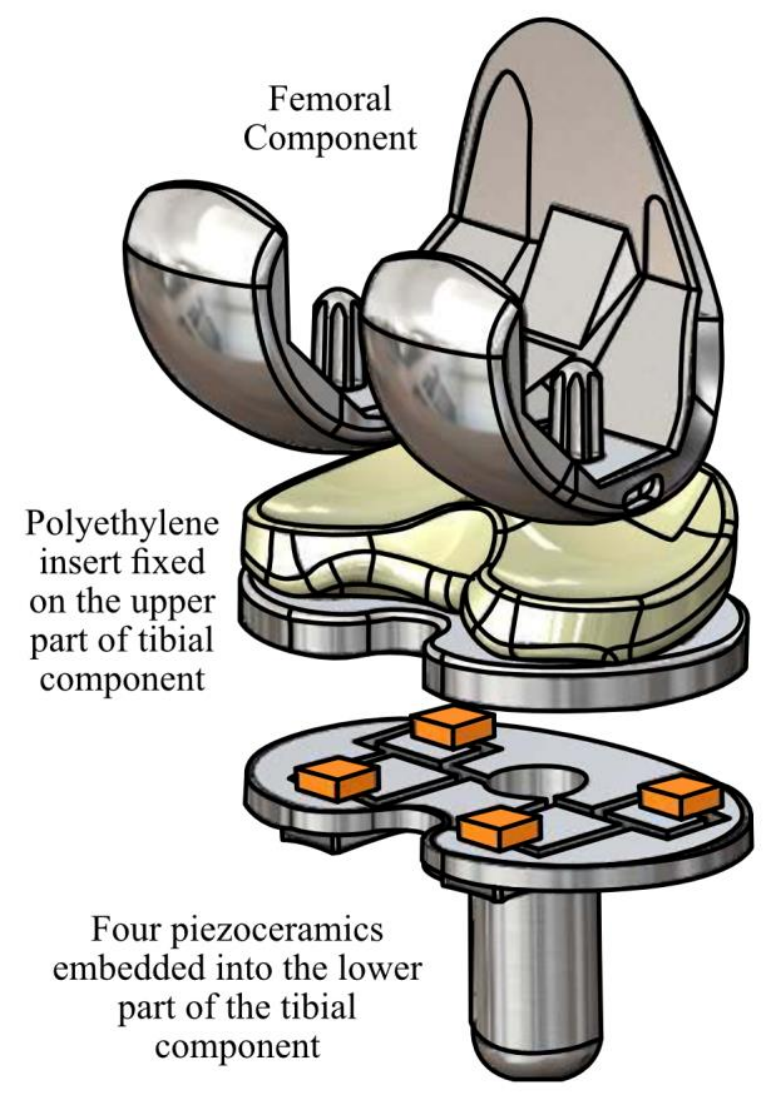

Figure 8. A knee implant design capable of telemetric measurement through usage of four load sensors imbedded in the base of the tibial component. Image adapted from (Almouahed et al., 2017).

being developed. We may parametrize geometry, loading conditions, and physical properties to better model the in vivo characteristics of an implant, and we typically model muscles and ligaments using actuators and loading profiles in the same manner as a joint simulator. The benefits of modeling are that they are significantly cheaper to produce, and allow us to generate results in a much shorter timeframe, before any part of a prototype must be manufactured. FEM allows us to implement simplifying assumptions in various parts of the models, so that we may reduce time-to-results by excising unimportant factors, and, in the case of simplifying a more complex model, validate our assumptions that those factors were indeed not important. 
One of the benefits of FEA models is that researchers may generate data that would otherwise be infeasible to measure. Joint mechanic data during dynamic motion is considerably easier to collect from a computational model when compared to experimental measurements, which would require an implant with built-in telemetric sensors and a laboratory equipped to utilize them (Figure 8). Models also offer us considerable speed gains when trying to model patient variability before implantation, and we are nearing the capability of producing patient-specific models which may aid in implant selection, customization, and placement. The main obstacle to this is procuring imaging scans with a high fidelity, and the subsequent process of image segmentation necessary to turn those scans into a computer aided design model that may be meshed using FEA techniques. Even with these challenges in place, it is much easier and faster to model individualized patient factors than it would be to, say, rapid prototype a patient-specific model for use in a joint simulator. Work is underway to utilize image recognition techniques, such as convolution neural networks to automate the image segmentation process (Burton II et al., 2019; Tack et al., 2018), and once that barrier is removed it is hoped that it will be possible to scan a patient's joint, quickly generate a model, and select the optimal implant geometry and surgical alignments to recreate the patient's previously healthy joint kinematic profiles.

The present thesis work utilizes an FEM model of an implanted knee, based on a previously developed model which was validated against a mechanical joint simulator (Baldwin et al., 2012; Fitzpatrick et al., 2014), as well as design of experiments and regression techniques. Additional background of these topics is provided in Chapter 3. 


\section{CHAPTER 3}

\section{TECHNICAL BACKGROUND}

This chapter gives a brief overview of the finite element method and detailed background on the use of interpolation shape-functions, as well as common methods of experimental design. Within the experimental design section, various experimental sampling methods and regression analysis techniques are described in sufficient detail, as well as the use of factor effect screening to reduce the number of input parameters within a model.

\subsection{Finite Element Methods}

The finite element method (FEM) is a numerical method for solving problems of engineering and mathematical physics. More specifically, it is a general discretization procedure of continuum problems posed by mathematically defined statements. The types of problems best suited to the use of FEM often require the use of partial differential equations and known boundary values for their analytical solution, which may be reduced down to algebraic equations when discretized. The procedure is to divide the continuum into a finite number of elements, where the behavior of each element is defined by a finite number of parameters, and the solution of the complete system as an assembly of its elements follows the same rules as those applicable to standard discrete problems which results in an ap- 
proximation to the continuum problem (Zienkiewicz et al., 2010). Like any tool in the engineer's arsenal, FEM encompasses a number of advantages and disadvantages, which are outlined in the following subsections.

\subsubsection{Advantages of FEM}

Discretizing a continuum into smaller elements has several advantages, including:

- Accurate representation of complex geometry

- Inclusion of dissimilar material properties which may vary throughout the continuum

- The safe simulation of potentially dangerous, destructive or impractical loading conditions and failure modes

- Comprehensive representation of the total solution, at any location, which leads to the

- Capture of local effects which may have been neglected in an analytical approach

- When the model is validated against an experiment, the ability to extrapolate existing experimental results using model parameters

- Relatively low investment of time and expertise compared to experimental methods

\subsubsection{Disadvantages of FEM}

There are some disadvantages, of course. Some of the limitations are:

- Uninformed solver/element selection may lead to inaccurate results 
- The solver calculations are susceptible to round-off errors

- Solutions are sensitive to mesh size and element types

- Leading commercial FEM packages are purely numeric, with no dimensional analysis safeguard in place

- Good engineering judgement and experimental validation still required for interpretation of results

While it can be challenging to develop a FEM model, the benefits listed above readily lend themselves to studies involving machine learning. Once an initial model is developed and parameterized, it is possible to generate a large number of simulations and training data for use as training inputs for statistical analysis and prediction. This use-case justifies their usefulness as a tool for the development of less resource intensive predictive models created through classical means. These classical methods often take sampled data and generate predictions through the use of basis interpolation functions, which in the FEM world are known as shape-function models.

\subsection{Shape Function Models}

Shape functions are a type of interpolation function used within finite element analysis to approximate the exact solution of field variables within a modeled continuum. They are a type of basis function which are used as the functional building blocks for individual discretized elements, which may themselves be connected together to describe a larger, more complicated continuum geometry. Since shape functions are themselves basis functions within a specific field, it is helpful to describe basis functions in general. 
Basis functions are the tools we use when modeling governing phenomena and fitting data. When modeling, we desire a mathematical description of a curve which fits data distributed over a continuum, such as time or space. We need this mathematical construct to be flexible and modular, because we need it to be adaptable between different data and phenomena. To accomplish this, we may choose to create linear combinations of elementary functions. The simplest example of a basis function is the family of polynomials, which have been used to model particle motion through the time continuum. The polynomial functions consist of the powers of $t$, which may be shifted or scaled individually, and summed together to model more complex behavior. An example of a polynomial basis function is

$$
f(t)=a_{0} \theta_{0}\left(t-b_{0}\right)+a_{1} \theta_{1}\left(t-b_{1}\right)+\cdots+a_{k} \theta_{k}\left(t-b_{k}\right)
$$

where scaling and shifting are accomplished by the $a_{i}$ and $b_{i}$ terms, respectively, and the different $\theta_{i}$ terms represent different powers of $t$, though not necessarily the $i^{\text {th }}$ power of $t$. Other examples of basis functions include the Fourier basis, which is used to model periodicity, and an extension of the polynomial basis, named the spline basis, which offers significantly more control by imposing constraints at the boundaries of piecewise polynomial domains. These basis functions may be applied in the modeling of any continuum using FEA, where they serve to smooth and interpolate the transition between adjacent elements, and we adopt the shape function moniker when working within this field.

While the traditional definition of shape functions relates to the interpolation of the transitions between elements and their respective nodes, the biomedical engineering community has adapted the term to include any form of basis function which estimates the field 
variables associated with finite element models, when model geometries are used as input factors (Fitzpatrick et al., 2011a, 2011b). Typically, if the interpolation is done outside the finite elements themselves, researchers say they are using statistical shape functions. In summary, shape functions are basis functions which are used to link geometry to function by interpolating and approximating continuum phenomena. Within the context of this thesis work, the term shape function will describe the statistical prediction of key joint mechanics using FEA simulations as the training and validation data sets. The production of these sets and the shape function models used on them is performed using common techniques of experimental design.

\subsection{Experimental Design}

During an experiment, we change one or more input variables (factors) in order to observe the effects of those changes on one or more output variables (responses). The design of experiments is the act of planning experiments such that the response data obtained can be analyzed to yield valid and objective conclusions across the experimental design space. It is infeasible to test every combination of factors, so judicious choice of factor combinations is necessary to allow us enough data to be able to model the response variables as continuous functions. The choice of factor combinations is known as factor sampling, and the continuous equations used to model the response variables often take the form of linear or quadratic polynomial basis functions. 

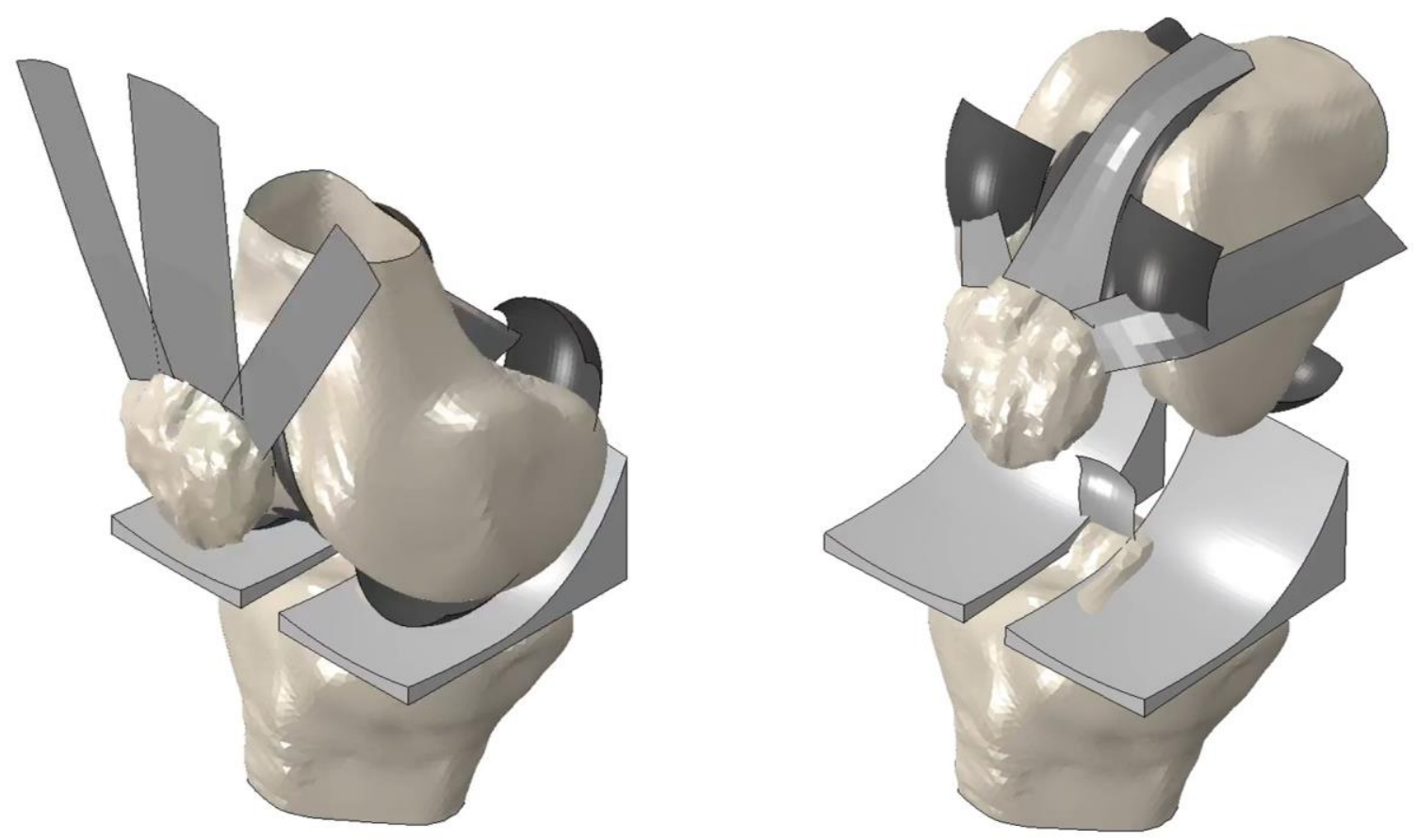

Figure 9. Factorial designs only produce good models when we can collect accurate data, which was not possible in the case of this implant design (left), which used the tibial insert as a launch-ramp to dislocation (right). Other sampling methods do not require such a large number of samples and have a better spread throughout the design space, mitigating the loss of accuracy when missing a single data point.

\subsubsection{Sampling Methods}

Methods of sampling an experimental design space include random and non-random designs, where randomized samples are the preferred method to eliminate selection bias. Simple random sampling is performed by assigning every possible factor value an equal probability of selection. This is implemented by sampling a uniform distribution between the high and low factor values, and repeating the process until the desired number of samples are chosen. The weakness of this method is that there are no guarantees in place to ensure that the sampled points do not cluster near each other, leaving important parts of the design space unsampled. To combat this, it is possible to systematically increase the probability of sampling within certain ranges of factors, where a non-uniform distribution 

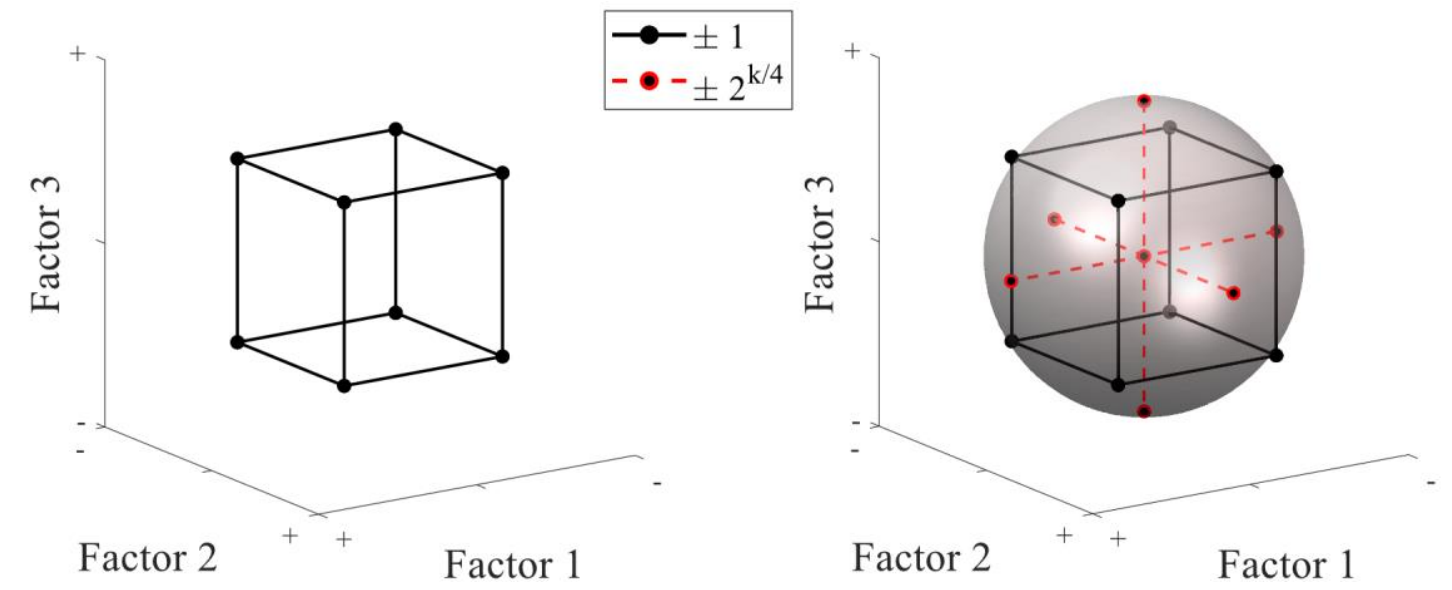

Figure 10. An example of a full factorial (left) and central composite (right) design spaces for an experiment with three factors. A full factorial design includes every combination of factors set to high and low levels, encoded to be \pm 1 . To extend a factorial design with $\boldsymbol{k}$ factors into a central composite design, we add additional "star points" at a distance $\pm 2^{k / 4}$ away from the mean factor value. In two and three dimensions, these additional points will circumscribe a circle or sphere with the original points.

is assigned to each factor. This is known as proportional-to-size sampling, and the primary challenge with this sampling method is selecting a criterion to choose other distributions which will not introduce selection bias. A method which guarantees samples throughout the design space without the possibility of introducing bias is desirable. Some such desirable methods are factorial or central-composite, and Latin square designs.

Factorial designs take the range of factors, select evenly spaced intervals within those ranges, and sample every combination of factor landing on the interval boundaries. This method can be extended to a central-composite design by including additional sample points where all factors are held averaged except for one, which is then sampled a distance below and above the normal ranges (Figure 10). This is performed for every factor, and generally leads to a more accurate response model throughout the sampled design space. These methods perform very well when it is feasible to collect good data, but scientists and 

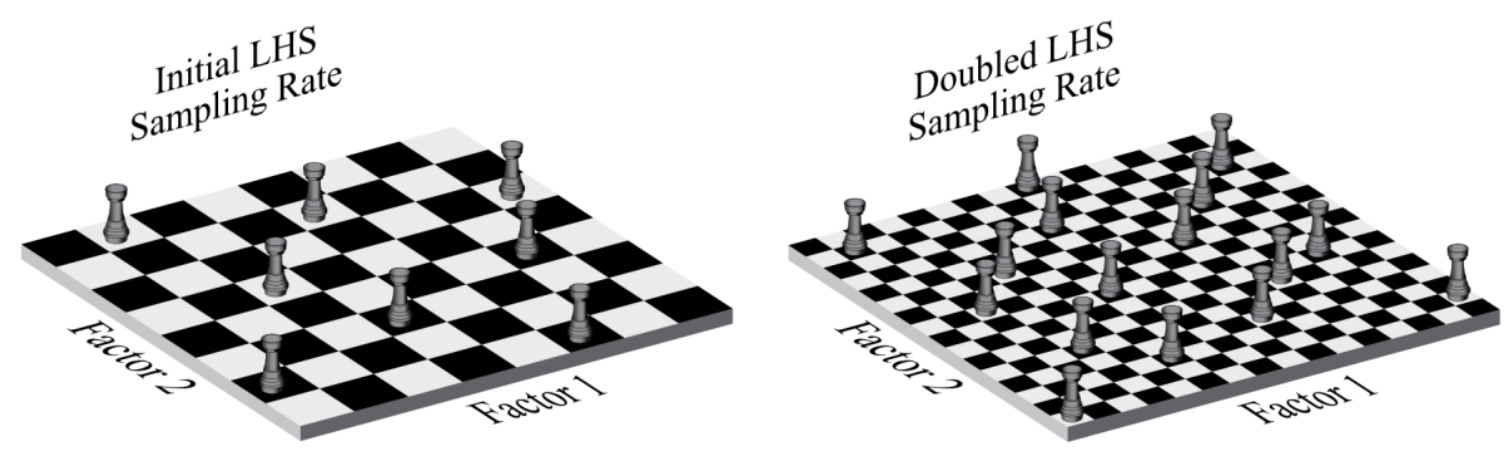

Figure 11. Sampling a two-factor design space using a Latin square is analogous to placing rooks on a chessboard such that none of them are able to capture each other. One of the benefits of LHS is being able to select the number of samples independently from the number of factors; we can subdivide the chess board as little or as much as we want.

engineers generally develop models for the widest range of factors possible, and it is often the case that the extreme parameter combinations will result in experiments that are not possible to implement (Figure 9). Additionally, these methods can result in an extremely large sample set, and are typically avoided when the number of factors is high. For example, a full-factorial design with five factors would require 32 experimental runs, and a central-composite design would require 42 runs, both excluding replications. A sampling method which can guarantee sampling throughout the design space without an exponential increase in sample size is the Latin square method.

Latin square sampling allows for sample size selection independent from the number of factors examined. A design space with two factors will form a square. If we subdivide that square into evenly spaced smaller square elements, one element for each possible sampling point, then our sample forms a Latin square if and only if there is only one sample in each row or column (Figure 11). With this method, we control the number of elements within the design space, and this allows us to sample the entire design space while imposing a limit on sample size. This process is analogous to placing many rooks on a chess board, 
without any of the rooks being in position to attack the others, but we're not limited to the standard number of tiles on the board (Figure 11). It is still possible that some sample points may cluster together, so computer algorithms have been developed to perform this process iteratively, maximizing the distance between sample points, or reducing sample correlation. This process extends to as many dimensions as number of factors dictates, and is known as Latin hypercube sampling when more than three factors are required. Due to the independence of sample size and coverage, as well as the computational efficiency, within this thesis work, Latin hypercube is the preferred sampling method, and the method of predicting design response is regression analysis.

\subsubsection{Regression Analysis}

Regression analysis is the statistical process of estimating the relationship between the factor and response variables, often using basis functions. Regression analysis takes many forms, but in all cases a function of the factor variables called the regression function is to be estimated, and this function estimates the average response to varying factor levels (Devore, 2012). For this thesis work, multiple linear regression is the chosen method of predicting joint mechanic response variables, and this term warrants an explanation. A multiple linear regression equation takes the simplest form of

$$
\hat{y}=\beta_{0}+\beta_{1} f_{1}\left(x_{1}\right)+\beta_{2} f_{2}\left(x_{2}\right)+\cdots+\beta_{n} f_{n}\left(x_{n}\right),
$$

where the equation is linear in coefficients, but not necessarily linear in predictors. The predictors are free to be transformed by any function we choose, but are most often modeled as polynomials. When using this model, the estimated response value $\hat{y}$ is assumed 
to be a scalar value, rather than a vector of values, but the increasing speed of computers allows us to create individual regression functions for each scalar element of a vector. There are several estimation techniques for fitting the $\beta_{i}$ parameters, but ordinary least squares methods, where the overall solution minimizes the sum of the squares of the residual errors, are the most common. With the freedom to transform the $x_{i}$ predictors, the method is a powerful tool for response predictions, but care must be chosen to select a predictor order (or function) that is able to capture the observed response.

\subsubsection{Predictor Order}

When selecting a linear regression function within the polynomial basis, there are terms that account for the primary factor effects, and we have the option of including secondary interaction effects. These interaction effects are modeled as products of the main factors, and it is trivial to create a very large number of these interaction terms. For example, if we select a model that is quadratic in three predictors, while including every interaction term, we arrive at

$$
\begin{gathered}
\hat{y}=\beta_{0}+\beta_{1} x_{1}+\beta_{2} x_{2}+\beta_{3} x_{3}+\beta_{12} x_{1} x_{2}+\beta_{13} x_{1} x_{3}+\beta_{23} x_{2} x_{3}+\beta_{11} x_{1}^{2} \\
+\beta_{22} x_{2}^{2}+\beta_{33} x_{3 .}^{2}+\beta_{123} x_{1} x_{2} x_{3}
\end{gathered}
$$

When looking at this equation with only three factors, it is clear that regression models will rapidly increase in complexity when including interactions, although, it should be noted that the $\beta_{123} x_{1} x_{2} x_{3}$ term is often excluded. Likewise, we may choose to omit the inclusion of some of these other interaction terms, and iterative methods exist that will attempt to include or exclude each term based on a selection criterion, such as minimizing 
the root-mean-square error of the modeled vs. measured response, but they become computationally expensive when modeling a response with numerous factors. These algorithms are known as stepwise fitting of linear models. Including stepwise methods, the linear regression models that are examined within the present thesis work use a polynomial basis, and are called linear, linear with interactions, pure quadratic, and quadratic with interactions.

An example of a linear model with differing predictor order:

Linear

$$
\hat{y}=\beta_{0}+\beta_{1} x_{1}+\beta_{2} x_{2}+\beta_{3} x_{3}
$$

Linear with interactions

$$
\begin{aligned}
\hat{y}=\beta_{0}+\beta_{1} x_{1} & +\beta_{2} x_{2}+\beta_{3} x_{3}+\beta_{12} x_{1} x_{2}+\beta_{13} x_{1} x_{3}+\beta_{23} x_{2} x_{3} \\
& +\beta_{123} x_{1} x_{2} x_{3}
\end{aligned}
$$

Pure quadratic

$$
\hat{y}=\beta_{0}+\beta_{1} x_{1}+\beta_{2} x_{2}+\beta_{3} x_{3}+\beta_{11} x_{1}^{2}+\beta_{22} x_{2}^{2}+\beta_{33} x_{3}^{2}
$$

Quadratic with interactions

$$
\begin{gathered}
\hat{y}=\beta_{0}+\beta_{1} x_{1}+\beta_{2} x_{2}+\beta_{3} x_{3}+\beta_{12} x_{1} x_{2}+\beta_{13} x_{1} x_{3}+\beta_{23} x_{2} x_{3}+\beta_{11} x_{1}^{2} \\
+\beta_{22} x_{2}^{2}+\beta_{33} x_{3}^{2}+\beta_{123} x_{1} x_{2} x_{3}
\end{gathered}
$$



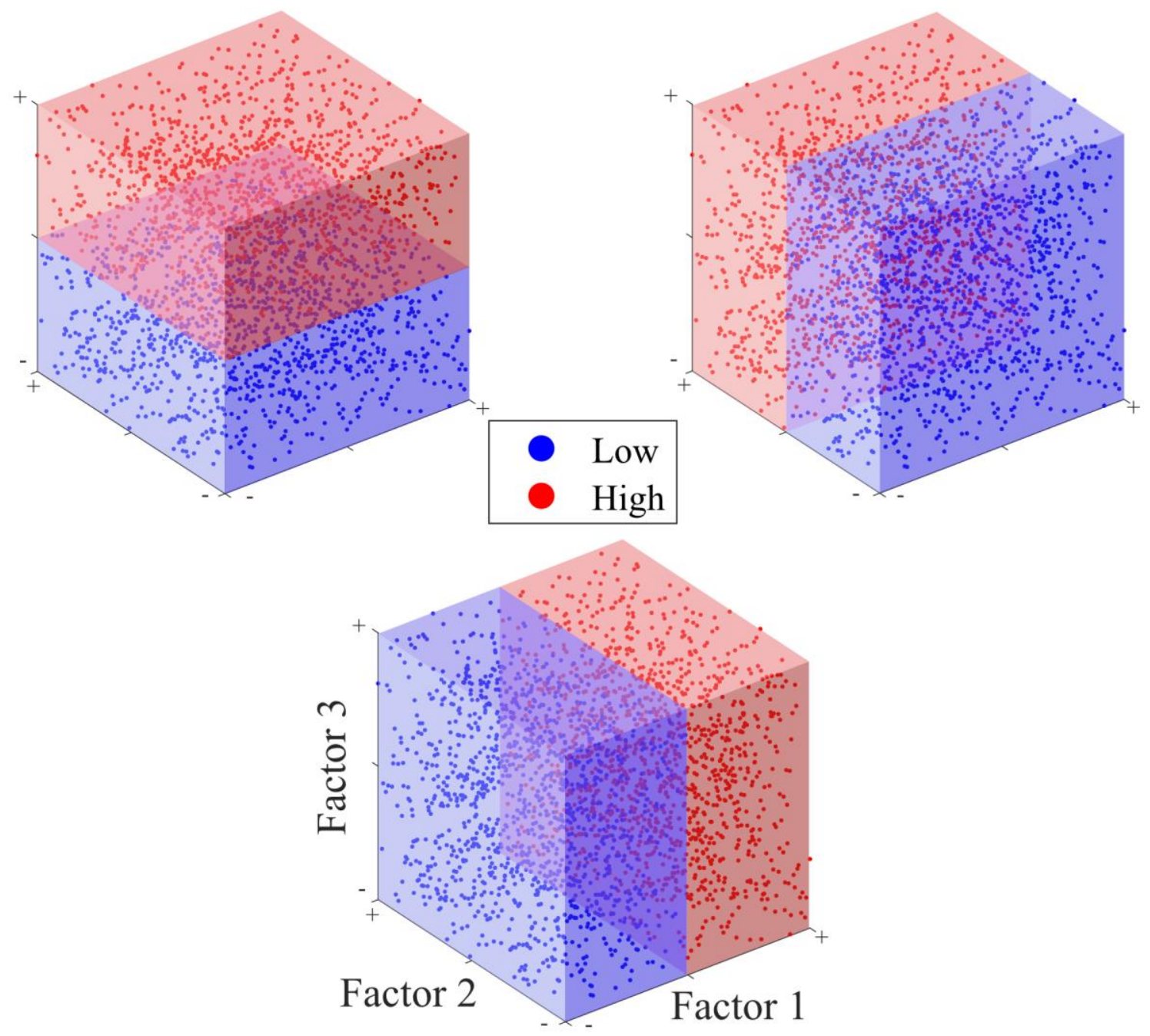

Figure 12. An example of the low and high factor groupings used when performing factor effect screening on a three-factor design space. The difference between average response of the high and low factor values is used for ranking the importance for each individual factor.

When viewing the preceding equations, it becomes apparent that reducing the number of factors is of the upmost importance during model development. This process of selecting important factors is performed using a sensitivity analysis, with the simplest method being factor effect screening. 


\subsubsection{Factor Effect Screening}

When designing an experiment, there is an infinite number of potential factors to choose from, but many will have negligible effect, with only a subset of these factors actually influencing the modeled response. A method is required to quantify the sensitivity of the response to changes in the factor levels, and one such method is factor effect screening. To perform factor effect screening, you first calculate the response variable of interest for every factor combination included within the chosen sample set. Next, you group the responses based on whether a single factor is above, or below its average value (Figure 12). Once this is completed, we may reduce the many response values to a pair of statistical metrics, often the average, where we have a statistic for the high and low factor values. Finally, the magnitude of the difference between these values are taken to be representative of the sensitivity of the response to this one factor. When we repeat this for every factor, we have quantitative data to compare sensitivities and decide which factors are most important for study. While this process is simple for a single response variable, additional complexity arises when studying multiple response variables, as it is unlikely that the studied responses are dimensionally consistent. In these cases, it becomes important to nondimensionalize the output response variables so that they may be aggregated together into a combined sensitivity.

The preceding treatments of the finite element methods, shape-function models, and experimental design offer sufficient background for us to understand the methods used within the present thesis work, which are detailed in Chapter 4. 


\section{CHAPTER 4}

\section{MANUSCRIPT "DEVELOPMENT OF A STATISTICAL SHAPE-FUNCTION MODEL OF THE IMPLANTED KNEE FOR REAL-TIME PREDICTION OF JOINT MECHANICS"}

The following manuscript has been published in the journal of biomechanics (see Page 16), and contains a succinct description of our usage of finite element methods to create a predictive model for the joint mechanics of an implanted knee performing a deep knee bend (DKB). The training set was sampled using Latin hypercube methods, factor effect screening was performed to reduce the number of factors by two, and linear regression was performed to generate predictive models for output mechanics for the duration of the DKB. The various polynomial linear regression models were compared, with the quality of results compared against a test set of simulations using an average root-mean-square error criterion.

\subsection{Introduction}

Outcomes of total knee arthroplasty (TKA) are dependent on surgical technique, patient variability, and implant design. Non-optimal design or alignment choices may result in undesirable contact mechanics and joint kinematics, including poor alignment, instability, and reduced range of motion. Some of the concerns specifically affecting posterior stabilized designs are bone resection, cam position, post wear and breakage, patellar 
clunk, and anterior-posterior (A-P) mid flexion instability (Scuderi et al., 2012). Patient satisfaction rates range from $75 \%$ to $92 \%$, with primary complaints being residual pain and limited function, including difficulty kneeling, squatting, and climbing stairs after TKA, resulting in only $22 \%$ of patients rating their surgical outcomes as 'excellent' (Choi and Ra, 2016). Implant design and surgical alignment are modifiable factors that have potential to improve patient outcomes. There is a need for robust implant designs that can accommodate patient-specific sources of variability. Real-time prediction of TKA joint mechanics under variable design and/or alignment conditions would facilitate incorporating these factors into patient-specific surgical planning strategies to optimize post-operative joint mechanics.

Prior research has assessed joint mechanics outcomes for TKA procedures both experimentally and computationally, with FE methods used extensively in both deterministic and probabilistic studies using available patient data, as well as data generated through statistical means (Galloway et al., 2012). Studies focusing on implant design (Willing and Kim, 2011), surgical decisions (Kessler et al., 2008; Thompson et al., 2012), and subjectspecific factors (Dhaher and Kahn, 2002; Elias et al., 2010; Mesfar and Shirazi-Adl, 2005) have been conducted, while other work has quantified the relative contributions of each source with respect to total variability (Fitzpatrick et al., 2012). Recent work has identified geometric parameters of the articular surface that cause a competing duality between optimal contact pressure and knee kinematics (Ardestani et al., 2015). The authors noted that femoral and tibial distal radii, femoral and tibial posterior radii, and femoral coronal radius were all parameters that affected contact mechanics and kinematics simultaneously, and 
gave pairs of parameters that would optimize either contact pressure or knee kinematics at minimal detriment to the other.

While these past efforts have given valuable insight into predicting surgical outcomes following TKA, there are inefficiencies that limit their usefulness to individual patients within the clinical setting. In vitro experiments are often expensive, which limits the number of designs or alignments that may feasibly be evaluated. Finite element (FE) methods combined with probabilistic analysis provides an effective platform to investigate coupled interactions between knee design parameters, surgical choices, and patient-specific variability (Ardestani et al., 2015). However, time, effort, and expertise is required to perform each simulation, which imposes a barrier to adoption of traditional FE methods for development of targeted TKA treatments on a patient-specific basis. A statistical model linking design and alignment parameters to joint mechanics outcomes with real-time response could have substantial utility during surgical planning to guide the optimal surgical choices for an individual.

Our objective was the development of a statistical shape-function model (SFM) of a posterior stabilized implant knee to instantaneously predict output mechanics in a resource efficient manner. Finite element methods were combined with Latin hypercube sampling (LHS) and regression analysis to produce modeling equations relating nine implant design and six surgical alignment parameters to tibiofemoral (TF) joint mechanics outcomes during a deep knee bend (DKB) activity. Initially, only design parameters were modified and resulting TF contact mechanics, kinematics, and soft tissue loads were predicted. Once the initial prediction algorithms were tuned, a separate set of models with averaged design parameters and varied surgical parameters was developed. Finally, an 

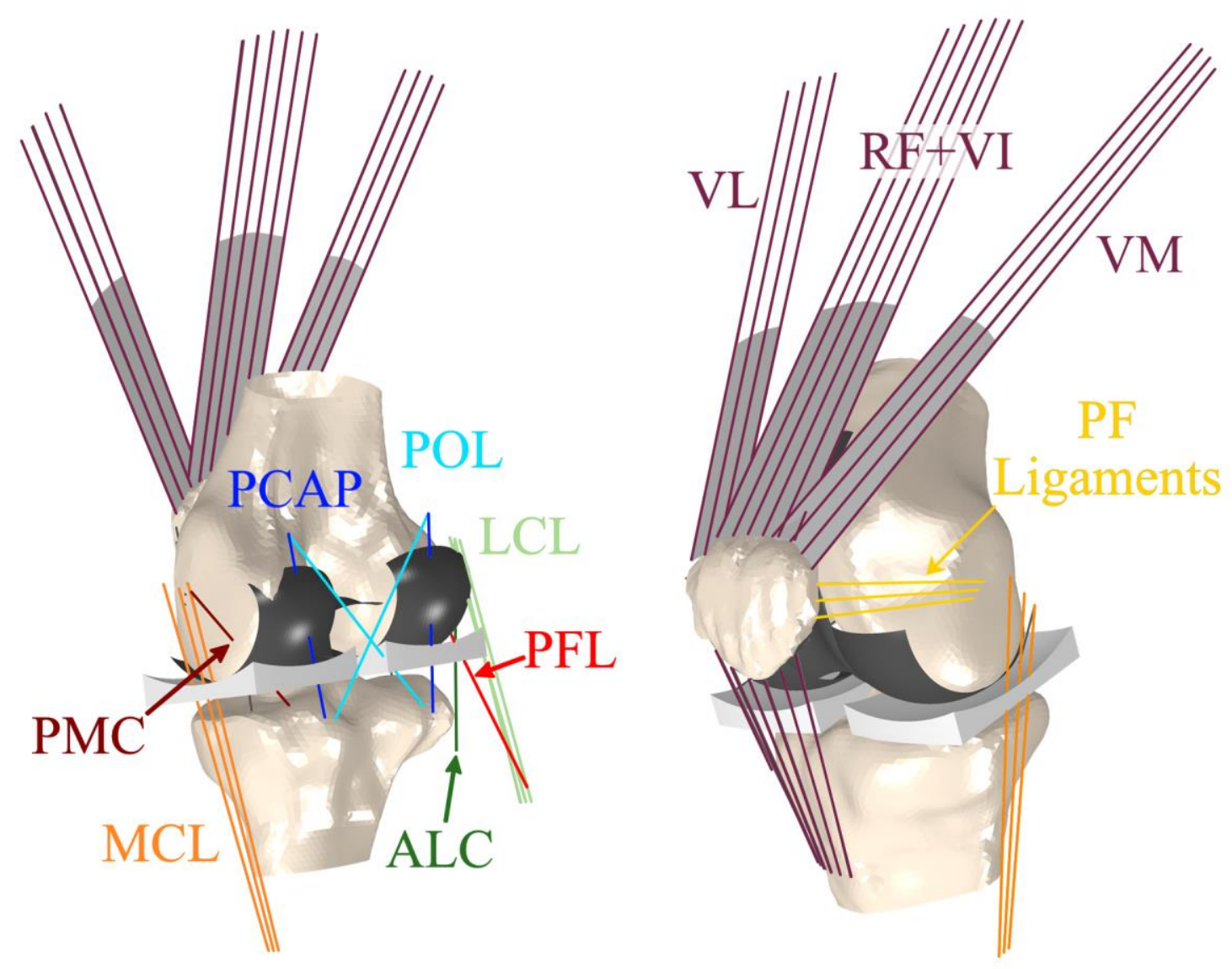

Figure 13. Finite element model of knee joint TKA. Ligaments modeled in this simulation included medial and lateral collateral ligaments (MCL, LCL), anterior lateral capsule (ALC), popliteofibular ligament (PFL), postero-medial capsule (PMC), posterior capsule (PCAP), and posterior oblique structures (POL). The quadriceps muscle, which was separated into rectus femoris plus vastus intermedius $(\mathrm{RF}+\mathrm{VI})$, vastus lateralis $(\mathrm{VL})$ and vastus medialis $(\mathrm{VM})$ bundles, was represented by 2-D fiber-reinforced membrane elements. Patellofemoral ligaments and patellar ligament (PL) were also included.

SFM was developed where both design and surgical parameters were varied, and joint mechanics were instantaneously predicted from the resulting model.

\subsection{Methods}

The FE model in this study was based on a previously developed model of an implanted posterior-stabilized (PS) knee model (Fitzpatrick et al., 2014, 2012). In brief, the 
model consisted of femoral, tibial, and patellar bones and implants, patellar tendon, quadriceps, and PF and TF ligaments (Figure 13). Each ligament was represented by non-linear tension-only spring elements, with reference strains and linear stiffness parameters adopted from prior work (Baldwin et al., 2012). Ligaments included medial and lateral collateral ligaments (MCL, LCL--separated into anterior, medial and posterior bundles), anterior lateral capsule (ALC), popliteofibular ligament (PFL), posteromedial capsule (PMC), medial and lateral posterior capsule (MPCAP, LCAP), and medial and lateral posterior oblique structures (MPOL, LPOL). The quadriceps muscle, which was separated into rectus femoris plus vastus intermedius $(\mathrm{RF}+\mathrm{VI})$, vastus lateralis $(\mathrm{VL})$ and vastus medialis $(\mathrm{VM})$ bundles, was represented by two-dimensional (2-D) fiber-reinforced membrane elements to allow for contact and wrapping in deep flexion, with line of action for each bundle based on cadaveric data (Farahmand et al., 2004). Sensors in the FE model, represented using connector elements, were used to connect each tibial implant surface (separated into medial, lateral and post components) to the underlying tibial bone. These sensors were used to record the 6-DOF loads acting on each surface. Bone and components were modeled as rigid bodies, for computational efficiency, with contact between the components defined using a pressure-overclosure relationship (Halloran et al., 2005). A coefficient of friction between the femoral component and polyethylene components of 0.04 was applied (Godest et al., 2002; Hashemi and Shirazi-Adl, 2000).

Knee loads and muscle forces were applied using hinge, translational, or cylindrical (allowing both rotation and translation) mechanical actuators, implemented in our FE through force- or moment-driven connector elements. To create a 6-DOF TF joint, quadriceps load was applied to the RF+VI, VL and VM actuators, vertical load was applied at the 
hip, anterior-posterior (A-P) force was applied to the femur, with femoral internal-external (I-E) rotation constrained, medial-lateral (M-L) translation free, and knee flexion determined by a balance of vertical hip and quadriceps load. I-E and varus-valgus $(\mathrm{V}-\mathrm{V})$ torques were applied to the tibia, with the remaining tibial DOFs constrained. The patella was kinematically unconstrained in all 6-DOF, with constraint provided by the patellar ligament (PL), patellofemoral ligaments (PFL), and quadriceps (Baldwin et al., 2012).

The knee flexion profile for a deep knee bend simulation was adopted from video recordings of five patients with telemetric implants during a knee bend activity (Heinlein et al., 2007; Kutzner et al., 2010). TF compression, A-P, I-E and V-V loading profiles were extracted from the published telemetric data of these same patients. Flexion and joint loading profiles were averaged across the five subjects to create representative TKA profiles. These were used as load and kinematic targets in the FE simulation. Sensors in the FE model were used to measure knee flexion angle, compressive, A-P, I-E, and V-V loads at the tibiofemoral joint throughout the dynamic knee flexion simulation where the knee was flexed from full extension to $100^{\circ}$ flexion. A proportional-integral (PI) control system was implemented in the model through an Abaqus/Explicit user subroutine to apply the external loads required to match the target joint loading and flexion profiles. External loads were applied through translational and rotational connectors to apply a vertical load to the hip, A-P force to the femur, I-E and V-V torques to the tibia, and muscle forces to the quadriceps. The quadriceps force was updated via the control system to match flexion angle, while loads in the vertical hip, femoral A-P, tibial I-E and tibial V-V connectors were updated to match their associated $\mathrm{TF}$ joint loading profile. 
Table 1. Parameter ranges used in training and test data sets for design and surgical parameter sets. Implant geometry was parameterized using nine variables with ranges based on the geometric domain of commercially available TKA components. Six surgical alignment predictors were included, with ranges chosen to capture those of both mechanically and kinematically aligned knee replacements.

Parameter

Design Set

Femoral Distal Radius (mm)

Femoral Posterior Radius (mm)

Femoral Coronal Radius (mm)

Tibial Insert Anterior Conformity

Tibial Insert Posterior Conformity

Tibial Insert Coronal Conformity

Trochlear Groove Angle (degrees)

Trochlear Groove Offset (mm)

Cam Radius (mm)

Surgical Set (degrees)

Tibial Insert Vr(+ve)-Vl Alignment

$-9.40$

0.00

$-0.80$

$-0.20$

0.00

High

50.0

50.0

Tibial Insert I(+ve)-E Alignment

Tibial Insert Posterior Slope

Femoral Vr(+ve)-V1 Alignment

Femoral I(+ve)-E Alignment

Femoral F(+ve)-E Alignment
5.00 

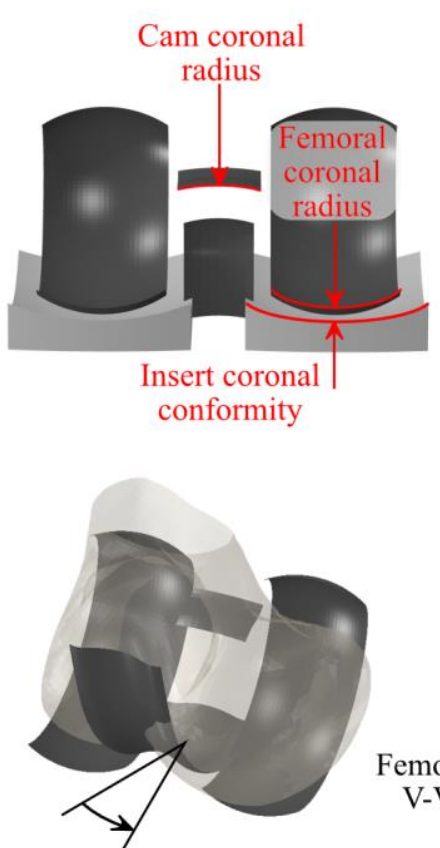

Femoral I-E

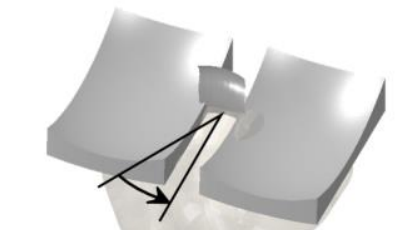

Tibial I-E
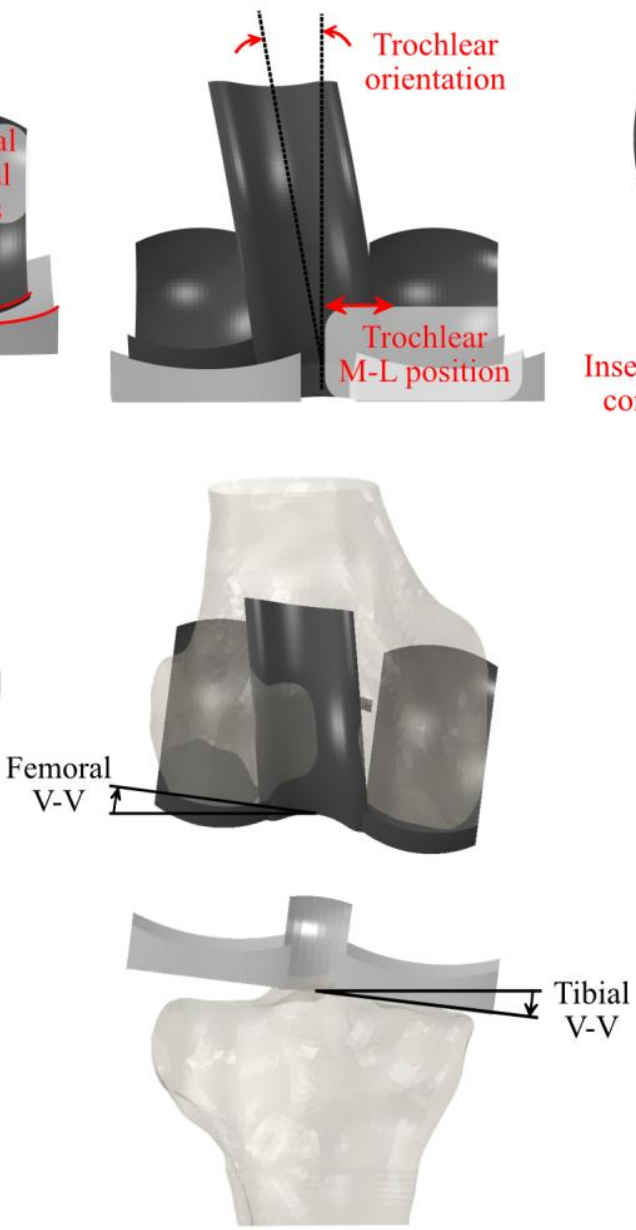
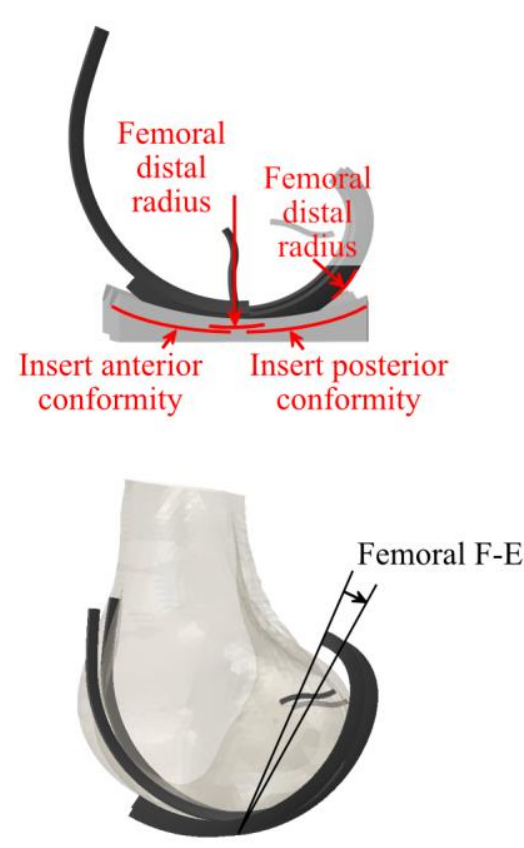

Figure 14. Design and surgical alignment parameters, with all surgical alignments shown positive. Implant geometry (red) was parameterized using nine variables: femoral condyle distal, posterior and coronal radii, tibial insert anterior, posterior and coronal plane conformity, trochlear orientation and M-L position, and coronal plane curvature of the cam mechanism. Six surgical alignment predictors (black) included: tibial insert and femoral implant V-V and I-E alignment, femoral F-E alignment, and tibial insert slope, with ranges chosen to capture those of both mechanically and kinematically aligned knee replacements.

Implant geometry was parameterized using nine variables: femoral condyle distal, posterior and coronal radii, tibial insert anterior, posterior and coronal plane conformity, trochlear orientation and M-L position, and coronal plane curvature of the cam mechanism (Figure 14). Ranges of design parameters were based on the geometric range of commercially available TKA components. Six surgical alignment predictors included: tibial insert and femoral implant V-V and I-E alignment, femoral F-E alignment, and tibial insert slope, 
with ranges chosen to capture those of both mechanically and kinematically aligned knee replacements (Howell and Hull, 2012; Theodore et al., 2017) (Table 1). A previously developed MATLAB script (Fitzpatrick et al., 2012) was used to automatically generate surface geometry of the femoral medial and lateral condyles, trochlear and cam geometries, and tibial insert medial and lateral condyles and post geometries from the nine design parameters. The surfaces were meshed as quadrilateral elements, with an average element edge length of $1 \mathrm{~mm}$ (Halloran et al., 2005), and a dome-shaped patellar button design was used in all analyses.

Linear and quadratic regression analyses were used to develop the shape-functions within this study. Regression analysis is used to estimate the relationships between multiple predictor variables, or factors, and one or more response variables. There exist many methods of sampling the predictor experimental design space including random, factorial or central-composite, and Latin square designs. Latin hypercube sampling (LHS) allows for sample size selection independent from the number of factors examined. For this study, the models combining design and surgical parameters included 13 factors, which would require 4123 samples to produce a 1/6 fractional central composite design. As such, computationally efficient LHS was selected for this study. An initial sample rate of $20 \mathrm{FE}$ simulations per predictor variable was selected, followed by halving the rate to 10 simulations per predictor to compare the accuracy of predictions with reduced design space sampling. 
Table 2. Predicted outputs organized by functional groupings used in factor effect screening. Four groups were created for the tibiofemoral joint, while a single group collected all outputs for the patellofemoral joint.

Functional Group

Tibiofemoral Joint Loads

Tibiofemoral Contact Mechanics

Tibiofemoral Kinematics

Ligament Elongations and Muscle Forces

Patellofemoral Mechanics

\section{Response Outputs}

A-P force, compressive force, $\mathrm{V}-\mathrm{V}$ torque, I-E torque

Contact area, pressure, and center of pressure

A-P translation, I-E rotation, $\mathrm{V}-\mathrm{V}$ rotation

PCL, PMC, MCL, POM, ALC, POL, LCL, and PL elongations; PFL elongation and forces, and vasti muscle force

M-L, S-I, and A-P contact force; Contact area, pressure, and center of pressure; All clinical translations and rotations, PL elongation and force 

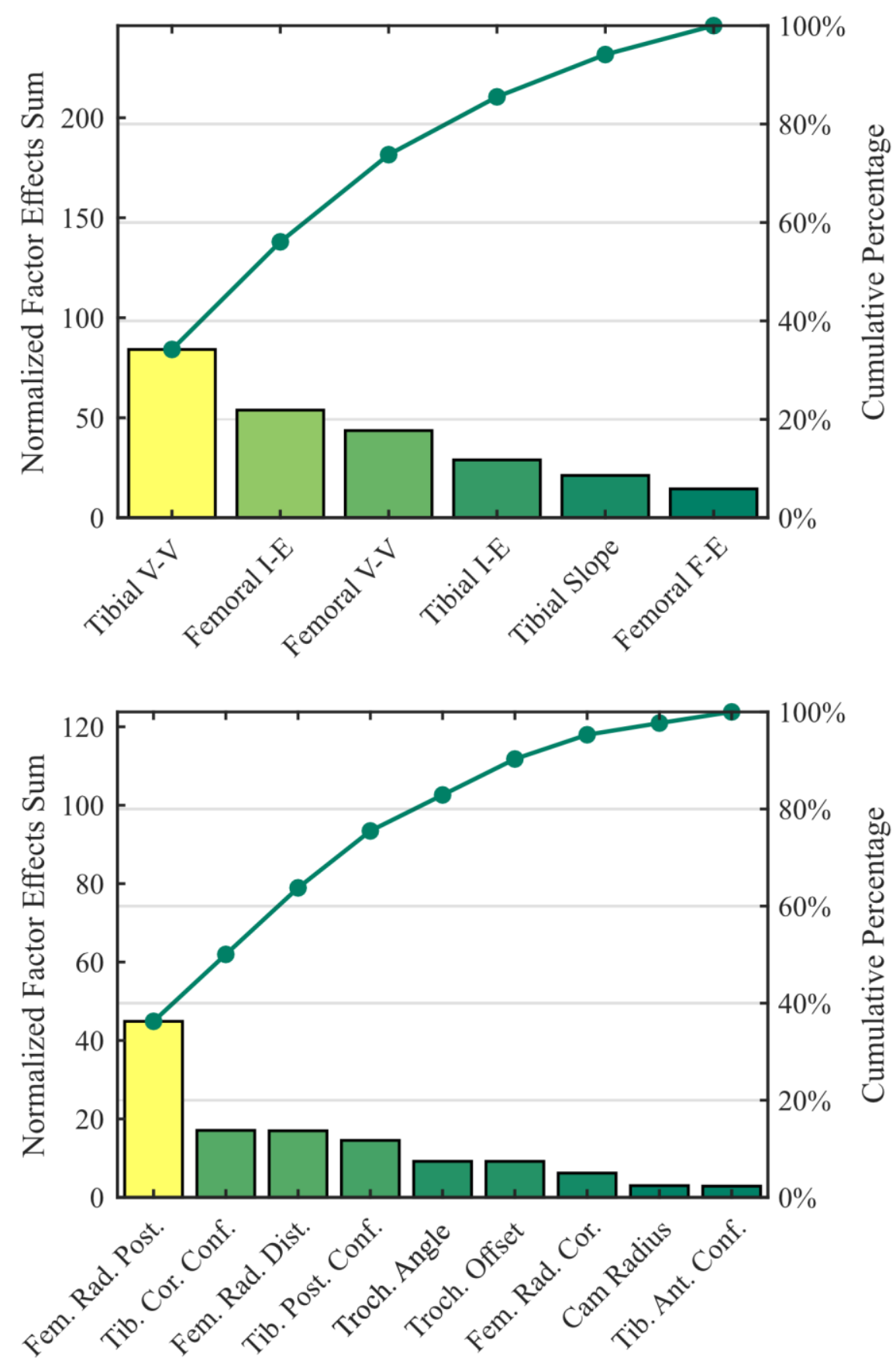

Figure 15. Pareto charts justifying the removal of femoral F-E alignment and cam radius parameters from the combination set, developed from factor effect screening analysis for the surgical alignment (top) and design geometry (bottom) parameters. Factor effects screening was performed by dividing output results into low and high factor blocks, where each single predictor variable was categorized as low when set below its average value, and high when set above average. For every combination of output and factor, the magnitude of the difference between the high block and the low block was taken and normalized by dividing by the average. 
For the surgical alignment and design parameter combination set, the initial rate of 20 FE simulations per predictor resulted in a sampling of 300 simulations. In order to reduce the computational cost of this set, the least critical parameters were eliminated. This was accomplished using factor effect screening analysis, which was performed by dividing output results into low and high factor blocks, where each predictor variable was categorized as low when set below its average value, and high when set above average. For every combination of output and input, the magnitude of the difference between the high block and the low block were collected into functional groups of outputs, including: joint loads, contact mechanics, kinematics, and ligament elongations (Table 2). These factor sensitivities were used to populate Pareto charts (Figure 15), which were used to remove implant F-E surgical alignment and cam radius design parameters from the combined set.

With sets including nine design geometry parameters, six surgical alignments, and a combined set of 13 parameters, the initial sampling rate produced 180, 120, and 260 simulations for the design, surgical, and combined sets, respectively. Following this initial sampling, the half-sized samples produced 90 simulations for the design, 60 for the surgical, and 130 for the combination sets. For all sets of data, linear regression was performed using MATLAB's fitlm function, across all response variables for every increment of time. A DKB was simulated over three seconds with a time increment of 0.025 seconds, resulting in 121 regression models ( 1 at each time point) for each response variable. The initial sets of data included enough simulations to allow for regression models specified as having quadratic predictors with interaction terms included $(\mathrm{Q}+\mathrm{I})$, but the reduction in simulations for the final set forced the use of linear predictors during the fitting process. To investigate and uncouple sample rate from predictor order, additional data was generated using the 
large sets with linear predictors, and all half-sized sets using MATLAB's stepwiselm function, which produces linear models using a forward-and-backwards stepwise algorithm. This algorithm uses a residual sum-of-squares criterion for adding or removing terms, and was initialized with linear predictors. Reported response variables were combined into functional groups, including joint loads, contact mechanics, kinematics, and ligament elongations (Table 2).

To quantify the predictive capability of the models for each of design, surgical, and combination sets, 100 additional parameter combinations were generated for each, using randomized parameter values sampled from uniform distributions of each factor range. This number was selected such that the central limit theorem (CLT) would be satisfied by a wide margin. 30 samples are required by the CLT to ensure that calculated RMS error estimations approach their true mean errors. The parameter values were input into each regression model, and the resulting outputs were compared to those of an FE simulation using the same parameters. This comparison was quantified using root mean square (RMS) errors averaged across all randomized test simulations. To measure the relative size of the RMS errors to their respective response variables, the average ranges of $95 \%$ confidence intervals for each parameter, and the percentage of the range represented by the RMS errors were reported as average normalized RMS errors. 
Table 3. Average normalized RMS errors for all outputs (\%). Across all three parameter sets, doubling the sample rate while using quadratic predictors with interactions reduced errors by an average of $30.1 \%$. Further investigation of sensitivity to predictor order was performed on the design set, due to it having the largest reduction in error with increasing order.

Mean Normalized RMS Error $(\%)$

Sample Rate (Simulations / Parameter) Predictor Order Surgical Design Combined

10

Linear

4.13

7.80

9.42

10

Stepwise Linear

4.13

7.18

9.67

20

Linear

3.99

7.38

8.97

20

Linear with Interactions

$--$

6.54

6.77

20

Pure Quadratic

$--$

2.79

4.89

7.49

\subsection{Results}

DKB predictions were performed for each parameter set, with calculated average normalized RMS errors producing similar trends for each parameter set (Table 3). For the design parameters, when using linear predictors and a rate of 10 samples per parameter, the average normalized RMS error was $7.8 \%$. This error was reduced to $7.4 \%$ when doubling the sampling rate, and further reduced to $4.9 \%$ when specifying Q+I predictors. The surgical parameter set behaved similarly - average normalized RMS error was $4.1 \%$ with 
the linear predictors and smaller sample rate, which reduced to $4.0 \%$ when doubling the sample rate, and was minimized to $2.8 \%$ when increasing predictor order. Combining the design and surgical parameters resulted in an average normalized RMS error of $9.4 \%$ when sampled at 10 simulations per parameter using linear predictors. Using 20 simulations per parameter decreased the error to $9.0 \%$, and including Q+I predictors further reduced the average normalized RMS error to $7.5 \%$. Across all parameter sets, doubling the sample rate while using $\mathrm{Q}+\mathrm{I}$ predictors reduced errors by an average of $30.1 \%$. Due to the considerable gains in computational efficiency and for brevity, only specific output results from the smaller sample size of 10 simulations per parameter using linear predictors are presented here. The design set had 47 out of a total of 56 outputs below $15 \%$ normalized RMS error, with the highest error being $25.7 \%$. The surgical set had average normalized RMS errors for nearly all 56 outputs below 15\%, with the lone exception of lateral area, which resulted in a predictive error of $16.8 \%$. For the combination set, 43 of the 56 outputs were below $15 \%$ average normalized RMS errors, with all errors falling below $28.8 \%$. Results for each parameter set may be specified by functional groups, including: patellar, ligament elongation, kinematics, joint loads, and contact mechanics outputs (Figure 16). Key results for individual sets are detailed below. 


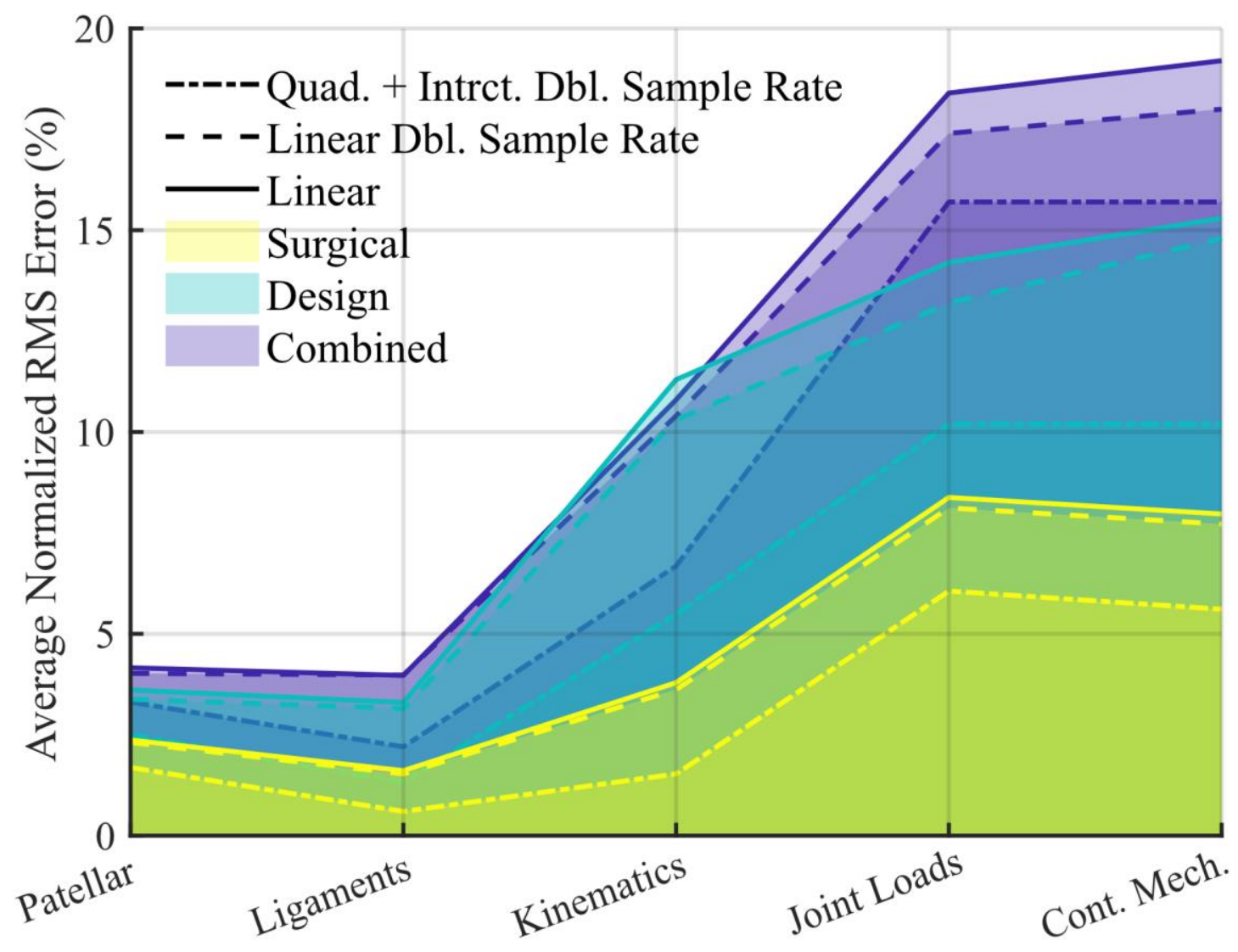

Figure 16. Overlayed area plots of average normalized RMS error by functional group. Within each set of parameters, highest errors are associated with linear predictors using half-sized samples, and are reduced by keeping linear predictors while doubling the sampling rate, then minimized using doubled sample rate with quadratic predictors with interaction terms. Joint loads and contact mechanics functional groups produced larger differentiation between each set, with the small sample rate with linear predictor sets showing average differences in errors of $5.6 \%$ and $5.0 \%$ when going from surgical to design, and design to combination sets, respectively.

\section{$\underline{4.5 .3}$ Patella}

Patellofemoral kinematics, contact mechanics, and joint forces represented one functional group. For this group, mean normalized RMS errors, averaged by group, were below 5\% across all parameter sets, with errors of $2.4 \%, 3.6 \%$, and $4.2 \%$ for surgical, design, and combined sets, respectively. For the surgical set, every output scored a mean normalized RMS error below 5\%, while both the design and combination set included error 

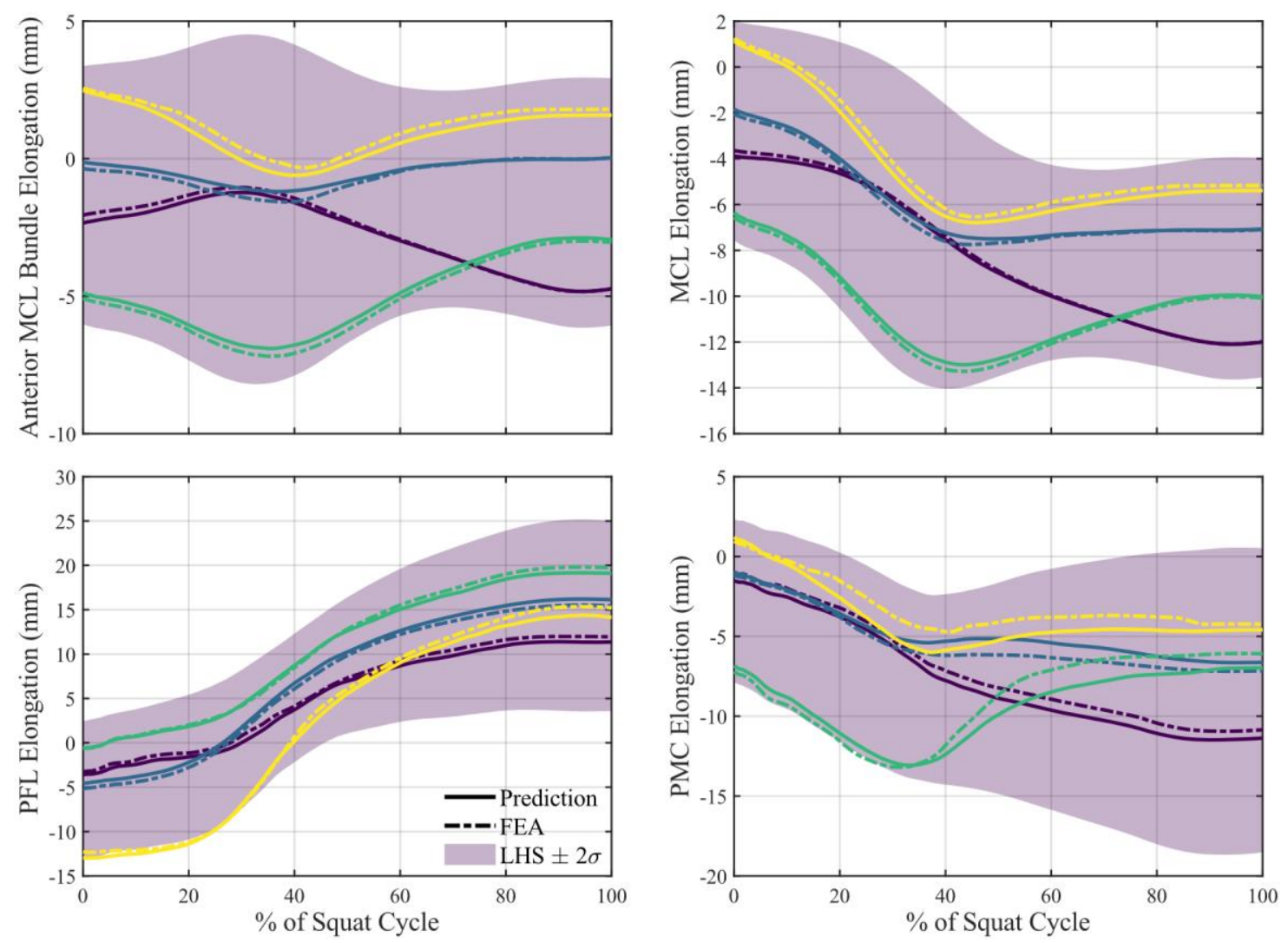

Figure 17. Select results from the ligament elongation functional group, from four randomly generated knees within the combination test set using a LHS sampling of 130 simulations with linear predictors. This functional group had normalized mean RMS errors, averaged by group, below $5 \%$ across all parameter sets, with errors resulting in $1.61 \%, 3.30 \%$, and $3.97 \%$ for the surgical, design, and combined sets, respectively.

terms below $10 \%$, except for the M-L center of pressure, at $10.2 \%$ and $10.8 \%$ for the design and combined sets, respectively. A-P and S-I rotation, M-L and compressive A-P contact forces were accurately predicted, with mean normalized RMS errors below $2.5 \%$ for all parameter sets.

\subsubsection{Ligament Elongation and Muscle Forces}

The ligament group included MCL, LCL, PFL, PMC, POM, POL, and ALC elongations and vasti and PFL forces. The remaining forces developed in the ligaments were 

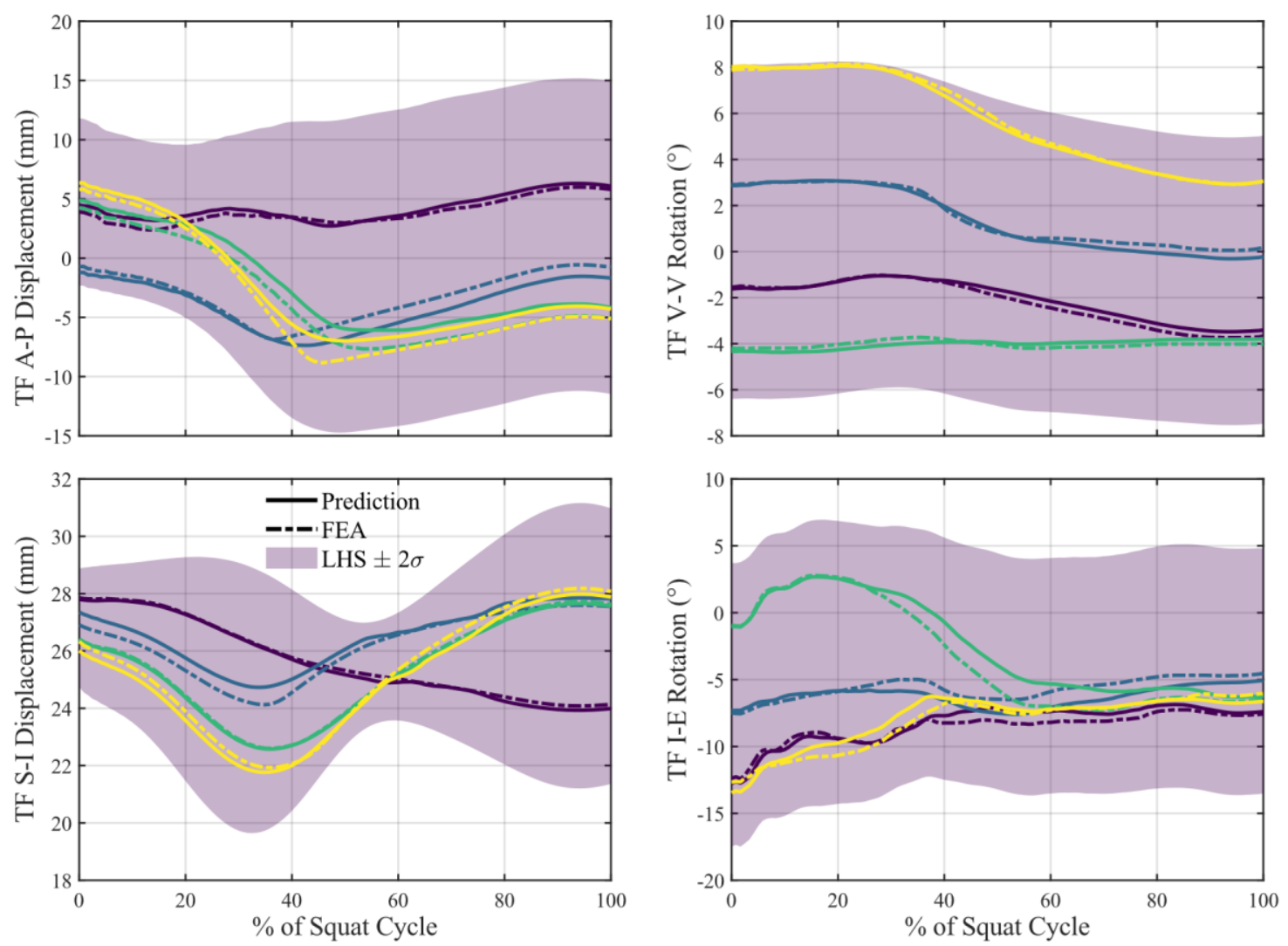

Figure 18. Prediction of kinematic results from four randomly generated knees for tibiofemoral A-P and S-I displacement, and I-E and V-V rotation of the combination parameter set using 10 simulations per parameter with linear predictors. Kinematics predictions of the TF joint resulted in normalized mean RMS errors, averaged by group, of $3.78 \%$ for the surgical, $11.3 \%$ for the design, and $10.8 \%$ for the combination parameter sets.

omitted due primarily to large domains of inactivity during the DKB; only a small subset of geometries produced forces, skewing all predictions for these omitted outputs. This group also had normalized mean RMS errors, averaged by group, below 5\% across all parameter sets, with errors resulting in 1.6\%, 3.3\%, and 4.0\% for the surgical, design, and combined sets, respectively. For the combined set, the highest mean normalized RMS error resulted from MCL elongation at $8.8 \%$. Prediction of forces developed within the PFL resulted in an error of $4.2 \%$, while vasti muscle force predictions contained an error of $3.2 \%$ (Figure 17). 


\subsubsection{Tibiofemoral Kinematics}

Kinematics predictions of the TF joint resulted in normalized mean RMS errors, averaged by group, of $3.8 \%$ for the surgical, $11.3 \%$ for the design, and $10.8 \%$ for the combination parameter sets. For the surgical set, all mean normalized RMS errors were less than $6.3 \%$. All outputs of the design set, apart from M-L translation, fell below a mean normalized RMS error of $11 \%$. The design set exception, M-L translation, had an error of 25.7\%. For the combination set, predicted mean normalized RMS errors for S-I and A-P translations, and V-V rotation fell below 10\%, while M-L translation and I-E rotation had prediction errors of $15.5 \%$ and $19.0 \%$, respectively (Figure 18).

\subsubsection{Tibiofemoral Joint Loads}

The TF joint loads functional group contained eight outputs, including: A-P and compressive forces, as well as V-V and I-E torques for both medial and lateral condyles of the tibial implant. For the surgical set, normalized mean RMS joint load errors, averaged by group, were only $8.4 \%$, whereas the design set resulted in an error of $14.2 \%$, and the combined set had the largest error of $18.4 \%$ for this group. For the combination set, all contact forces were predicted with mean normalized RMS errors less than $15 \%$ except for the medial A-P joint force, which scored 20.5\% (Figure 19). At the other end of the range, medial and lateral tibial insert joint torques scored mean normalized RMS errors between $21.3 \%$ and $28.8 \%$. 

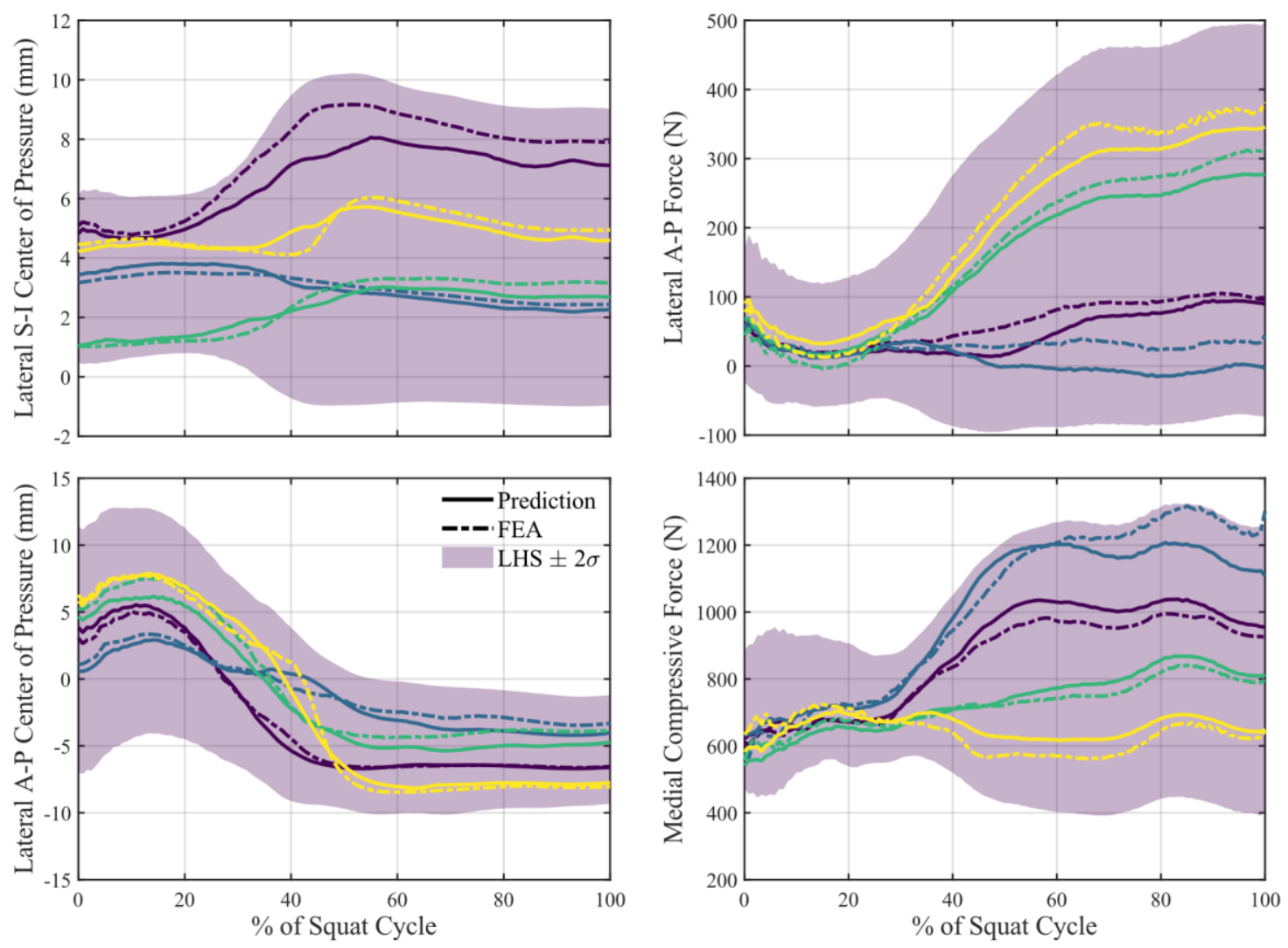

Figure 19. Representative contact mechanics and joint loads prediction results from the combination set, using a sample size of $\mathbf{1 3 0}$ simulations and linear predictors for regression analysis. Four randomly generated knees were selected from each functional group. From the contact mechanics group, the surgical set had a normalized mean RMS error, averaged by group, of $7.97 \%$, while the design set scored $15.3 \%$, and the combination set resulted in an error of $19.2 \%$. For the surgical set, normalized mean RMS joint load errors, averaged by group, were only $8.38 \%$, whereas the design set resulted in an error of $\mathbf{1 4 . 2 \%}$, and the combined set had the largest error of $18.4 \%$ for this group.

\subsubsection{Contact Mechanics}

The contact mechanics functional group included contact areas and pressures, and center of pressure locations in the S-I, M-L, and A-P directions, from both the medial and lateral condyles. For these 10 outputs, the surgical set had a normalized mean RMS error, averaged by group, of $8.0 \%$, while the design set scored $15.3 \%$, and the combination set resulted in an error of $19.2 \%$. For the surgical set, all mean RMS errors were below $15 \%$, 
except for contact area on the lateral side of the tibial insert at $16.8 \%$. For the design set, contact pressures and their A-P locations were within mean normalized RMS errors of $7.9 \%$, with the remaining outputs falling between $15.3 \%$ and $24.2 \%$. For the combination set, contact pressures and A-P locations fell below mean normalized RMS errors of 13.4\%, with the rest of the error terms falling between $20.2 \%$ and $28.2 \%$ (Figure 19).

\subsection{Discussion}

The goal of this study was to generate a statistical model to predict contact mechanics and kinematic outcomes of the TKA joint during a DKB activity due to implant design and surgical alignment. Utilizing a set of five surgical alignment and eight implant geometry parameters, our most computationally efficient model (130 training FE simulations) is able to instantaneously predict outputs relating to patellar, ligament elongations, and kinematics functional groups with high precision, resulting in errors less than $12 \%$. Our model is also able to predict outputs from the contact mechanics and joint loads functional groups with an aggregate mean normalized RMS error below $20 \%$, and we have quantified the reduction in error as the result of doubling sample size and increasing predictor order.

While it was expected that the joint loads and contact mechanics functional groups would prove the most challenging to predict, it was interesting that the average normalized RMS error of the kinematics group was reduced when moving from the design to combination parameter sets. Furthermore, when increasing the predictor order to include Q+I terms, the largest reductions in error, $4.1 \%$ for the combination and $5.8 \%$ for the design sets, came from the kinematics functional group. These results suggest that the relationship between knee kinematics and design parameters may exhibit more nonlinearity than the 
other functional groups. It may be beneficial for future studies where kinematic prediction is critical to use sample sizes large enough for $\mathrm{Q}+\mathrm{I}$ predictors in the regression models.

For each parameter set within this study, FE simulations were performed in order to train the statistical model for two different sampling rates. Additionally, test simulations were also run to quantify the accuracy of the shape-function model predictions. Due to these requirements, over 1000 simulations were performed which necessitated a computationally efficient knee model, and a number of assumptions and simplifications were required. The removal of F-E surgical alignment and cam radius parameters in the combined set was based on factor-effect screening, and these results are likely activity-specific - a different activity may affect the relative influence of these parameters. The model created a physiological loading condition at the knee joint, but not in a perfectly physiological fashion. Only the quadriceps muscle was included, with net contribution from the rest of the muscles incorporated without direct representation. The vertical hip load primarily controlled the compressive load at the joint, the A-P force at the joint was controlled by application of an A-P force to the femur, and I-E and V-V torques were controlled by moments applied to the tibia. The loading condition represented in the FE model reflects a single knee flexion activity. While we have made efforts to ensure that this loading condition is a valid representation of kinematics and joint loads from a TKA patient performing this activity, there is likely wide variability in loads across the TKA population which have not been accounted for in this model. However, the shape function modeling framework presented in this study could be expanded to include easily measurable patient-specific parameters (e.g. weight, limb alignment, lower limb mechanics, etc.) in order to develop a SFM that can be customized to an individual patient. 
In the current study, the bounds of the parameters included our analysis are guided by current design and alignment strategies, and our predictions have only been validated within these bounds. To investigate a broader range of design and alignment parameter would likely require development of a new SFM which incorporated these extended ranges in the training set. We would expect our current model to perform well with small deviations outside our current ranges, but would likely have poorer performance with large deviations, particularly with combinations of parameters that may result in discontinuities in joint mechanics behavior such as edge loading or joint dislocation. An additional constraint of the shape-function model developed in this study is that is has been developed to predict results from finite element simulations, rather than in vivo data. However, as finite element simulation is becoming increasingly used and accepted in pre-clinical design phase of prospective devices, the approach described here has distinct benefit in reducing the time and computational resources required during this iterative phase.

The present framework could be utilized for implant design in an optimization pipeline such as that used by (Willing and Kim, 2012, 2011). These studies utilized single and multi-objective optimization methods to solve for an optimized design based on minimizing deviation from natural knee kinematics. Performing optimization using these techniques required iteratively solving of the objective function hundreds of times in order to minimize deviation from natural knee laxity data. Each solution of this function required an FE simulation, and the computational requirements to perform one entire optimization were reported as 10 days of calculations for one of these studies. Comparatively, the upfront computational cost to build the statistical SFM was 130 simulations, with each simulation taking 20 minutes to solve on a standard PC using a single CPU, for an initial time- 
savings of eight days. Once produced, the SFMs developed in this study are able to predict all output results from a simulation nearly instantaneously within a high degree of accuracy, resulting in computational requirements to produce a similar number of results measured in seconds, not days.

This work illustrates the efficacy of statistical models in pre-clinical design of TKA devices. The SFM generated instantaneous joint mechanics predictions using a small number of training simulations, making it ideally suited for integration into a design optimization pipeline. Such a tool may be used, for example, to optimize kinematic function to achieve more natural kinematics or minimize implant wear. Additionally, patient-specific parameters, such as limb alignment and weight, could be included in a similar optimization pipeline and integrated into surgical planning tools to guide clinicians in choosing alignments that optimize joint mechanics and kinematics outcomes. These tools may aid the engineering and clinical communities in improving patient satisfaction and surgical outcomes. 


\section{CHAPTER 5}

\section{CONCLUSIONS AND FUTURE DIRECTIONS}

During the course of this thesis work, multiple training sets of parameterized FEA simulations of the implanted knee performing a deep-knee-bend were produced. These simulations were then used to train statistical linear regression models to aid in the realtime prediction of key joint mechanics, without necessitating the creation of additional FEA simulations. Three parameter sets were investigated, one containing six surgical alignment parameters, another containing nine geometric design parameters, and a final set utilizing 13 combined parameters, which were selected using factor effect screening to determine the parameters which had greatest impact on the aggregated response variables. For each parameter set, two sampling rates and multiple choices of predictor order were investigated, with the resulting RMS errors quantified. For the largest set of parameters, with the lowest sampling rate and simplest predictor order, mean normalized RMS errors were kept below $20 \%$ for all functional groups.

To aid researchers and implant design teams in their future work, we have charted and tabulated the results of an additional factor significance study of the combined factor set in Appendix A. To test significance, the same high and low factor blocks as those from factor effect screening were used to develop two-sample t-tests with a significance level of $\alpha=0.05$. The appendix is sectioned by functional group, where each section begins with 
a chart ranking factors by significance. Higher ranked factors significantly influence a higher proportion of the functional group's response variables, and additional information is presented showing the percent of unique response variables that are captured as additional factors are included. Following the charts, lookup tables are provided to search for the specific factor-response combinations where significance was established. The first table in each subsection will be referenced by factor name, while the second will be referenced by response variable.

Following this, Appendix B and C are devoted to sensitivity studies of the Design and Surgical sets, respectively. These were included as a tool for future researchers interested in the two factors which were reduced by factor effect screening. The factor ranking significance plots were produced using identical methods, but each appendix includes only a single table, which outlines the response variables which were significant to the removed parameters. The entries are referenced by functional group, and the response variables for which the Cam Radius (Table 14) and Femoral F-E alignment (Table 15) parameters may be found there. The Cam Radius parameter was found to be significant to only five response outputs, with the only applicable functional groups being the TF contact mechanics and joint loads functional group. In contrast to this, the Femoral F-E alignment parameter was significant for 20 of the response variables, spanning every functional group. While this parameter was found to be significant to a larger number of outputs, it was still within the last three rankings for every functional group except the kinematics group, and only captured $20 \%$ of the TF contact mechanics and $40 \%$ of the TF joint mechanics groups. While $80 \%$ of the kinematic response variables were captured by F-E femoral alignment, this was a group that maintained approximately $10 \%$ average normalized RMS errors, and this 
shows that this was a good choice of factor for removal. The addition of these significance studies lends further credence to this framework as a design tool, and the authors believe the present thesis work would be an invaluable component to future optimization pipelines. However, this work was not without limitations, and there is always room for additional research and future work.

\subsection{Limitations}

Within the current thesis work, the range of parameters included in our analysis were guided by current design and alignment strategies, and our predictions have only been validated within these ranges. It is expected that our current model would perform well with small deviations outside the current ranges, but attempting parameter combinations which fall outside of these upper and lower bounds would require additional training simulations and the generation of a new statistical model. Additionally, our FEA model was only produced, and validated, using a simulated deep-knee bend; the shape-function can only hope to produce predictions as good as the FEA simulations themselves, which incorporate their own assumptions and errors into our shape-function model. While linear regression is viewed as a method which is easily explained, each equation contains at least 13 parameters, and at most over 200, for the combined factor set. This firmly plants our model in the phenomenological realm, as it would require a great deal of effort to explain the resulting equations in terms of the governing physics of the system. While these limitations exist, finite element simulations are becoming an increasingly relied upon design 
tool, and the described approach has the distinct benefit of reducing the computational resources required to get approximate results quickly, while laying the groundwork for future research into implant design.

\subsection{Future Work}

In the following sections, we outline some prospective methods to improve the shape-function models, as well as describing some of the work that went into ensuring this framework may be adapted to other joints and models, and detail the first prospective steps towards adapting the framework for inclusion within an optimization pipeline and graphical user interface.

\subsubsection{Shape-Function Model Improvements}

Linear regression was the prediction algorithm selected for use during the present thesis work, but the field of machine learning is an area of high research activity, with new models and methods coming out frequently. It is very likely that a newer, more robust fitting algorithm exists within this realm, and more research can be undertaken to select and quantify better models. The authors investigated an automated stepwise linear fitting model during this thesis work, where the program attempted to add or remove certain interaction effects in an iterative manner, but this method did not take into accounts the physics being modeled, and likely did not test the most important factors first. The ordering of testing in automated algorithms is important, because every method must include a criterion for abandoning further attempts. The option would exist to have a researcher supervise the stepwise models, but would only be feasible if the number of models were reduced. 
This could be facilitated by improving the algorithms to fit parameters based on an array of response data, rather than generating a new model for every individual time step. For our FEA model, this would reduce the number of linear models by 121 for each response. While this may be feasible to perform for this model, it would still represent a considerable amount of human supervision, and would reduce the ease of which the present framework could be adapted to other models; someone would need to supervise the process every time the FEA model was upgraded. Due to these shortcomings of the linear regression models used for the present thesis, it would be beneficial to investigate different automated models and methodologies that would allow us to assign heavier weights to the most important factors. Some work to quantify factor importance was already performed during factor effect screening, and these results would likely prove useful in this regard.

The authors performed factor effect screening in order to reduce the number of training simulations required to ensure balance between training costs and model accuracy, but the results of these screenings were never reintroduced as feedback into the training routines. It is not unreasonable to expect that prediction models exist which will allow us to assign importance weights to the response variables, or to prioritize the fitting of important factor coefficients when training a model. With minimal research into the field of machine learning, decision tree regression looks promising (Czajkowski and Kretowski, 2016; Quinlan, 1992; Wang and Witten, 1997). This form of regression begins with one factor selection, then assigns a minimal variance tipping point for the factor quantity, creating a high and low block that is not necessarily centered about the factor mean. Predictions are then created based on the parameter falling within the high or low level, and, if the prediction error maintains above a minimum error threshold, the process repeats for the 
next most important factor. Essentially, what the method is doing is dividing a global design space into increasingly smaller partitions, and continuing this process until we can fit the partitioned spaces with individual simplified models. It is entirely possible that the branches of a decision tree will stop before using every factor, just as it is possible for a factor to dominate the model when set within a certain range. This process may be repeated to obtain better results, using model ensembling methods, such as Random Forest Regression.

To perform random forest regression, we sample our training set multiple times, with replacement, and run decision tree regression on each sampled training set (Breiman, 2001). Once completed, the resulting models are weighted against a selected criterion and combined into one model. The main idea behind this method is that combining multiple estimations into one may possibly result in a better model, and it is likely to reduce outlier bias due to the replacement sampling involved; a sample with 'good' data will be weighted higher than the others, and the important data will likely be contained within multiple high weight samples.

\subsubsection{Additional Parameterized Joints}

During the course of this thesis, we developed a computational framework which allowed us to predict the instantaneous joint mechanics response for the implanted knee, which was selected due to the relatively low patient satisfaction rates, which are approximately $20 \%$ lower when compared to a total hip replacement (Scott et al., 2012; Tilbury et al., 2016). However, the present framework could readily be adapted to other implanted joints such as the hip, and many back-end development efforts were made to allow this. 
The source code is organized into three object classes, with 59 method functions, where an entire experimental setup may be run from a single 250 -line script. The program allows users to assign factor variable names, the high and low levels of the design matrix, and variable names and values for constants, as well. Next, the program creates a configurable design matrix allowing for factorial and central-composite designs, as well as Latin hypercube designs.

When it comes time to run the Abaqus simulations, the program contains the capabilities to set up an organized file system for tracking the various results, and uses a command line interface to run Abaqus simulations in parallel, with the number of Abaqus instances, and cores per instance assigned by the user. This process includes the training sets and the test sets. The program is capable of running a list of Python scripts to extract result data from an Abaqus output file, and aggregates the results into structures representing the training and test sets, while maintaining simulation identifiers and handling failed simulations gracefully. The user may select the important joint mechanics outputs using regular expressions, and use the program to collect these outputs into user-defined functional groups. Once the correct results are selected, the user may run a sensitivity analysis on the results and produce fitting models for the selected outputs. Errors may be calculated using a single method call, and the program can produce plots for every output and treatment within the testing set.

While such modularity and user defined control would not be necessary to complete the present thesis work, the intent was to provide a head-start for future research. The freedom to define factors and response variables at runtime will allow future researchers to adapt this framework to other Abaqus models and parameterized joints. By design, the 
majority of the work necessary to adapt the framework will take place in Abaqus input files, where the selected factors, constants, and response variables will need to be encoded into an Abaqus simulation. The primary concern when converting the present framework to a different joint model will be the mesh generation functions for our parameterized geometries. Forecasting the challenges that will arise when future researchers undertake this endeavor, but it is expected that large portions of the mesh generation functions may be used to develop new functions, as they essentially operate using radial curvature and sweptpath methods. In addition to developing this framework in anticipation of other joints and models, all of the runtime user-defined parameters will lend themselves to the future development of a graphical user interface (GUI).

\subsubsection{Graphical User Interface}

In its present form, this thesis work is purely an academic proof-of-concept, but we've developed it with commercial, clinical, and research applications in mind. Currently, the use of the program requires multiple steps and more programming knowledge than the average technician. Making the program viable for licensing to other users, companies, and research groups predicates the creation of a user-friendly GUI, which would allow the user to select factor levels, and generate relevant output geometry and joint mechanic plots in a fast and responsive interface. Creation of a functional prototype interface using MATLAB would take the author approximately two months of work, but this would only be the first step towards a GUI that would be useful to each of the preceding stakeholders. In order to create a truly useful GUI, the entire framework should be transferred to a portable language, such as Python or C. 
Once an initial GUI was developed, additional features could be created, such as an optimization component which would allow users to supply target response profiles and iteratively find the parameter combinations which would most closely match these targets. This module could use a similar selection criterion to our own RMS error reporting in order to select an optimal solution, and we could expand this capability to allow for selection of multiple target profiles. With the capability to interface with Abaqus through the command line baked in already, the user could be given the capability to run a simulation of the optimal solution parameters to validate the modeled results. Before running a simulation, an ideal GUI would generate a graphical representation of the geometric meshes, and perform the clinical rotations for fast user checking of results. 


\section{REFERENCES}

Abdel, M.P., Morrey, M.E., Jensen, M.R., Morrey, B.F., 2011. Increased Long-Term Survival of Posterior Cruciate-Retaining Versus Posterior Cruciate-Stabilizing Total Knee Replacements. (Cover story). J. Bone Joint Surg. Am. 93, 2072-2078. https://doi.org/10.2106/JBJS.J.01143

Addressing the Nation's Primary Care Shortage: Advanced Practice Clinicians and Innovative Care Delivery Models, 2018.

Aitchison, G.A., Hukins, D.W.L., Parry, J.J., Shepherd, D.E.T., Trotman, S.G., 2009. A Review of the Design Process for Implantable Orthopedic Medical Devices. Open Biomed. Eng. J. 3, 21-27. https://doi.org/10.2174/1874120700903010021

Ardestani, M.M., Moazen, M., Jin, Z., 2015. Contribution of geometric design parameters to knee implant performance: Conflicting impact of conformity on kinematics and contact mechanics. The Knee 22, 217-224. https://doi.org/10.1016/j.knee.2015.02.011

Askari, A., Gholami, T., NaghiZadeh, M.M., Farjam, M., Kouhpayeh, S.A., Shahabfard, Z., 2016. Hyaluronic acid compared with corticosteroid injections for the treatment of osteoarthritis of the knee: a randomized control trail. SpringerPlus 5. https://doi.org/10.1186/s40064-016-2020-0

Bade, M.J., Kohrt, W.M., Stevens-Lapsley, J., 2010. Outcomes Before and After Total Knee Arthroplasty Compared to Healthy Adults. J. Orthop. Sports Phys. Ther. 40, 559-567. https://doi.org/10.2519/jospt.2010.3317

Baldwin, M.A., Clary, C.W., Fitzpatrick, C.K., Deacy, J.S., Maletsky, L.P., Rullkoetter, P.J., 2012. Dynamic finite element knee simulation for evaluation of knee replacement mechanics. J. Biomech. 45, 474-483. https://doi.org/10.1016/j.jbiomech.2011.11.052

Barbour, K.E., Helmick, C.G., Boring, M., Brady, T.J., 2017. Vital Signs: Prevalence of Doctor-Diagnosed Arthritis and Arthritis-Attributable Activity Limitation - United States, 2013-2015. MMWR Morb. Mortal. Wkly. Rep. 66, 246-253. https://doi.org/10.15585/mmwr.mm6609e1

Barnes, C.L., Mesko, J.W., Teeny, S.M., York, S.C., 2006. Treatment of Medial Compartment Arthritis of the Knee: A Survey of the American Association of Hip and Knee Surgeons. J. Arthroplasty 21, 950-956. https://doi.org/10.1016/j.arth.2006.01.003 
Basalo, I.M., Chahine, N.O., Kaplun, M., Chen, F.H., Hung, C.T., Ateshian, G.A., 2007. Chondroitin sulfate reduces the friction coefficient of articular cartilage. J. Biomech. 40, 1847-1854. https://doi.org/10.1016/j.jbiomech.2006.07.007

Bonamici, S., 2016. H.R.34 - 114th Congress (2015-2016): 21st Century Cures Act [WWW Document]. URL https://www.congress.gov/bill/114th-congress/house-bill/34 (accessed 6.29.19).

Breiman, L., 2001. Random Forests. Mach. Learn. 45, 5-32.

https://doi.org/10.1023/A:1010933404324

Brosseau, L., Yonge, K.A., Welch, V., Marchand, S., Judd, M., Wells, G.A., Tugwell, P., 2003. Thermotherapy for treatment of osteoarthritis. Cochrane Database Syst. Rev. https://doi.org/10.1002/14651858.CD004522

Burton II, W.S., Sintini, I., Chavarria, J.M., Brownhill, J.R., Laz, P.J., 2019. Assessment of scapular morphology and bone quality with statistical models. Comput. Methods Biomech. Biomed. Engin. 22, 341-351. https://doi.org/10.1080/10255842.2018.1556260

Choi, Y.-J., Ra, H.J., 2016. Patient Satisfaction after Total Knee Arthroplasty. Knee Surg. Relat. Res. 28, 1-15. https://doi.org/10.5792/ksrr.2016.28.1.1

Colwell, C.W., Chen, P.C., D'Lima, D., 2011. Extensor malalignment arising from femoral component malrotation in knee arthroplasty: Effect of rotating-bearing. Clin. Biomech. 26, 52-57. https://doi.org/10.1016/j.clinbiomech.2010.08.009

Czajkowski, M., Kretowski, M., 2016. The role of decision tree representation in regression problems - An evolutionary perspective. Appl. Soft Comput. 48, 458-475. https://doi.org/10.1016/j.asoc.2016.07.007

DesJardins, J.D., Walker, P.S., Haider, H., Perry, J., 2000. The use of a force-controlled dynamic knee simulator to quantify the mechanical performance of total knee replacement designs during functional activity. J. Biomech. 33, 1231-1242. https://doi.org/10.1016/S0021-9290(00)00094-4

Devore, J.L., 2012. Probability and Statistics for Engineering and the Sciences, 8th ed. Brooks/Cole, Cengage Learning, Boston, MA 02210.

Dhaher, Y.Y., Kahn, L.E., 2002. The Effect of Vastus Medialis Forces on Patello-femoral Contact: A Model-based Study. J. Biomech. Eng. 124, 758-767. https://doi.org/10.1115/1.1516196

Dingell, J.D., 2007. H.R.3580 - 110th Congress (2007-2008): Food and Drug Administration Amendments Act of 2007 [WWW Document]. URL https://www.congress.gov/bill/110th-congress/house-bill/3580 (accessed 6.29.19). 
Elias, J.J., Kilambi, S., Cosgarea, A.J., 2010. Computational assessment of the influence of vastus medialis obliquus function on patellofemoral pressures: Model evaluation. J. Biomech. 43, 612-617. https://doi.org/10.1016/j.jbiomech.2009.10.039

Farahmand, F., Naghi Tahmasbi, M., Amis, A., 2004. The contribution of the medial retinaculum and quadriceps muscles to patellar lateral stability — an in-vitro study. The Knee 11, 89-94. https://doi.org/10.1016/j.knee.2003.10.004

Fitzpatrick, C.K., Baldwin, M.A., Clary, C.W., Maletsky, L.P., Rullkoetter, P.J., 2014. Evaluating knee replacement mechanics during ADL with PID-controlled dynamic finite element analysis. Comput. Methods Biomech. Biomed. Engin. 17, 360-369. https://doi.org/10.1080/10255842.2012.684242

Fitzpatrick, C.K., Baldwin, M.A., Laz, P.J., FitzPatrick, D.P., L. Lerner, A., Rullkoetter, P.J., 2011a. Development of a statistical shape model of the patellofemoral joint for investigating relationships between shape and function. J. Biomech. 44, 2446-2452. https://doi.org/10.1016/j.jbiomech.2011.06.025

Fitzpatrick, C.K., Baldwin, M.A., Rullkoetter, P.J., Laz, P.J., 2011b. Combined probabilistic and principal component analysis approach for multivariate sensitivity evaluation and application to implanted patellofemoral mechanics. J. Biomech. 44, 13-21. https://doi.org/10.1016/j.jbiomech.2010.08.016

Fitzpatrick, C.K., Clary, C.W., Laz, P.J., Rullkoetter, P.J., 2012. Relative contributions of design, alignment, and loading variability in knee replacement mechanics. J. Orthop. Res. 30, 2015-2024. https://doi.org/10.1002/jor.22169

Galloway, F., Worsley, P., Stokes, M., Nair, P., Taylor, M., 2012. Development of a statistical model of knee kinetics for applications in pre-clinical testing. J. Biomech. 45, 191-195. https://doi.org/10.1016/j.jbiomech.2011.09.009

Godest, A.C., Beaugonin, M., Haug, E., Taylor, M., Gregson, P.J., 2002. Simulation of a knee joint replacement during a gait cycle using explicit finite element analysis. J. Biomech. 9.

Greenwood, J.C., 2002. H.R.5651 - 107th Congress (2001-2002): Medical Device User Fee and Modernization Act of 2002 [WWW Document]. URL https://www.congress.gov/bill/107th-congress/house-bill/5651 (accessed 6.29.19).

Grood, E.S., Suntay, W.J., 1983. A Joint Coordinate System for the Clinical Description of Three-Dimensional Motions: Application to the Knee. J. Biomech. Eng. 105, 136144. https://doi.org/10.1115/1.3138397

Haider, H., Walker, P.S., 2005. Measurements of constraint of total knee replacement. J. Biomech., Knee Mechanics: An Update of Theoretical and Experimental Analyses 38, 341-348. https://doi.org/10.1016/j.jbiomech.2004.02.014 
Halawi, M.J., Jongbloed, W., Baron, S., Savoy, L., Williams, V.J., Cote, M.P., 2019. Patient Dissatisfaction After Primary Total Joint Arthroplasty: The Patient Perspective. J. Arthroplasty 34, 1093-1096. https://doi.org/10.1016/j.arth.2019.01.075

Halloran, J.P., Petrella, A.J., Rullkoetter, P.J., 2005. Explicit finite element modeling of total knee replacement mechanics. J. Biomech., Knee Mechanics: An Update of Theoretical and Experimental Analyses 38, 323-331. https://doi.org/10.1016/j.jbiomech.2004.02.046

Harkin, T., 2012. S.3187 - 112th Congress (2011-2012): Food and Drug Administration Safety and Innovation Act [WWW Document]. URL https://www.congress.gov/bill/112th-congress/senate-bill/3187 (accessed 6.29.19).

Hashemi, A., Shirazi-Adl, A., 2000. Finite Element Analysis of Tibial Implants — Effect of Fixation Design and Friction Model. Comput. Methods Biomech. Biomed. Engin. 3, 183-201. https://doi.org/10.1080/10255840008915264

Heidari, B., 2011. Knee osteoarthritis prevalence, risk factors, pathogenesis and features: Part I. Casp. J. Intern. Med. 2, 205-212.

Heinlein, B., Graichen, F., Bender, A., Rohlmann, A., Bergmann, G., 2007. Design, calibration and pre-clinical testing of an instrumented tibial tray. J. Biomech., Interaction of Mechanics and Biology in Knee Joint Restoration and Regeneration 40, S4-S10. https://doi.org/10.1016/j.jbiomech.2007.02.014

Howell, S.M., Hull, M.L., 2012. Kinematic Alignment in Total Knee Arthroplasty, in: Insall and Scott Surgery of the Knee. Elsevier, pp. 1255-1268. https://doi.org/10.1016/B978-1-4377-1503-3.00120-7

Jordan, J.M., 2015. An Ongoing Assessment of Osteoarthritis in African Americans and Caucasians in North Carolina: The Johnston County Osteoarthritis Project. Trans. Am. Clin. Climatol. Assoc. 126, 77-86.

Kessler, O., Patil, S., Colwell, C.W., D’Lima, D.D., 2008. The effect of femoral component malrotation on patellar biomechanics. J. Biomech. 41, 3332-3339. https://doi.org/10.1016/j.jbiomech.2008.09.032

Kutzner, I., Heinlein, B., Graichen, F., Bender, A., Rohlmann, A., Halder, A., Beier, A., Bergmann, G., 2010. Loading of the knee joint during activities of daily living measured in vivo in five subjects. J. Biomech. 43, 2164-2173. https://doi.org/10.1016/j.jbiomech.2010.03.046

Lane, N.E., Brandt, K., Hawker, G., Peeva, E., Schreyer, E., Tsuji, W., Hochberg, M.C., 2011. OARSI-FDA initiative: defining the disease state of osteoarthritis. Osteoarthritis Cartilage, OARSI FDA Initiative on issues related to clinical development programs for the treatment 19, 478-482. https://doi.org/10.1016/j.joca.2010.09.013 
Law, G.W., Lee, J.K., Soong, J., Lim, J.W.S., Zhang, K.T., Tan, A.H.C., 2019. Arthroscopic debridement of the degenerative knee - Is there still a role? Asia-Pac. J. Sports Med. Arthrosc. Rehabil. Technol. 15, 23-28. https://doi.org/10.1016/j.asmart.2018.11.003

Lawrence, R.C., Felson, D.T., Helmick, C.G., Arnold, L.M., Choi, H., Deyo, R.A., Gabriel, S., Hirsch, R., Hochberg, M.C., Hunder, G.G., Jordan, J.M., Katz, J.N., Kremers, H.M., Wolfe, F., National Arthritis Data Workgroup, 2008. Estimates of the prevalence of arthritis and other rheumatic conditions in the United States. Part II. Arthritis Rheum. 58, 26-35. https://doi.org/10.1002/art.23176

Maletsky, L.P., Hillberry, B.M., 2005. Simulating Dynamic Activities Using a Five-Axis Knee Simulator. J. Biomech. Eng. 127, 123. https://doi.org/10.1115/1.1846070

McDermott, K.W., Freeman, W.J., Elixhauser, A., 2017. Overview of Operating Room Procedures During Inpatient Stays in U.S. Hospitals, 2014: Statistical Brief \#233. Agency for Healthcare Research and Quality (US), Rockville (MD).

Mesfar, W., Shirazi-Adl, A., 2005. Biomechanics of the knee joint in flexion under various quadriceps forces. The Knee 12, 424-434. https://doi.org/10.1016/j.knee.2005.03.004

Murphy, L., Schwartz, T.A., Helmick, C.G., Renner, J.B., Tudor, G., Koch, G., Dragomir, A., Kalsbeek, W.D., Luta, G., Jordan, J.M., 2008. Lifetime risk of symptomatic knee osteoarthritis. Arthritis Rheum. 59, 1207-1213. https://doi.org/10.1002/art.24021

NIH Consensus Statement on Total Knee Replacement, 2003. . Presented at the NIH Consensus and State-of-the-Science Statements, U.S. Department of Health and Human Services, NIH.

Qian, S., Ge, S., Wang, Q., 2006. The Frictional Coefficient of Bovine Knee Articular Cartilage. J. Bionic Eng. 3, 79-85. https://doi.org/10.1016/S1672-6529(06)60011-5

Quinlan, J.R., 1992. LEARNING WITH CONTINUOUS CLASSES, in: In Proceedings AI'92 (Adams \& Sterling, Eds). World Scientific, Singapore, pp. 343-348.

Rogers, P.G., 1976. H.R.11124 - 94th Congress (1975-1976): Medical Device Amendments [WWW Document]. URL https://www.congress.gov/bill/94th-congress/housebill/11124 (accessed 6.29.19).

Scott, C.E.H., Bugler, K.E., Clement, N.D., MacDonald, D., Howie, C.R., Biant, L.C., 2012. Patient expectations of arthroplasty of the hip and knee. J. Bone Joint Surg. Br. 94-B, 974-981. https://doi.org/10.1302/0301-620X.94B7.28219

Scuderi, G.R., Bourne, R.B., Noble, P.C., Benjamin, J.B., Lonner, J.H., Scott, W.N., 2012. The New Knee Society Knee Scoring System. Clin. Orthop. Relat. Res. 470, $3-$ 19. https://doi.org/10.1007/s11999-011-2135-0 
Shetty, A.A., Tindall, A., Ting, P., Heatley, F.W., 2003. The evolution of total knee arthroplasty. Part III:: surface replacement. Curr. Orthop. 17, 478-481.

https://doi.org/10.1016/j.cuor.2003.09.005

Swanson, E.A., Schmalzried, T.P., Dorey, F.J., 2009. Activity recommendations after total hip and knee arthroplasty: a survey of the American Association for Hip and Knee Surgeons. J. Arthroplasty 24, 120-126. https://doi.org/10.1016/j.arth.2009.05.014

Tack, A., Mukhopadhyay, A., Zachow, S., 2018. Knee menisci segmentation using convolutional neural networks: data from the Osteoarthritis Initiative. Osteoarthritis Cartilage 26, 680-688. https://doi.org/10.1016/j.joca.2018.02.907

Theodore, W., Twiggs, J., Kolos, E., Roe, J., Fritsch, B., Dickison, D., Liu, D., Salmon, L., Miles, B., Howell, S., 2017. Variability in static alignment and kinematics for kinematically aligned TKA. The Knee 24, 733-744.

https://doi.org/10.1016/j.knee.2017.04.002

Thompson, W.R., Rubin, C.T., Rubin, J., 2012. Mechanical regulation of signaling pathways in bone. Gene 503, 179-193. https://doi.org/10.1016/j.gene.2012.04.076

Tilbury, C., Haanstra, T.M., Leichtenberg, C.S., Verdegaal, S.H.M., Ostelo, R.W., de Vet, H.C.W., Nelissen, R.G.H.H., Vliet Vlieland, T.P.M., 2016. Unfulfilled Expectations After Total Hip and Knee Arthroplasty Surgery: There Is a Need for Better Preoperative Patient Information and Education. J. Arthroplasty 31, 2139-2145. https://doi.org/10.1016/j.arth.2016.02.061

Total Knee Replacement - OrthoInfo - AAOS [WWW Document], 2019. URL https://www.orthoinfo.org/en/treatment/total-knee-replacement/ (accessed 6.3.19).

Van Norman, G.A., 2016. Drugs, Devices, and the FDA: Part 2: An Overview of Approval Processes: FDA Approval of Medical Devices. JACC Basic Transl. Sci. 1, 277-287. https://doi.org/10.1016/j.jacbts.2016.03.009

Varadarajan, K.M., Harry, R.E., Johnson, T., Li, G., 2009. Can in vitro systems capture the characteristic differences between the flexion-extension kinematics of the healthy and TKA knee? Med. Eng. Phys. 31, 899-906.

https://doi.org/10.1016/j.medengphy.2009.06.005

Walden, G., 2017. H.R.2430 - 115th Congress (2017-2018): FDA Reauthorization Act of 2017 [WWW Document]. URL https://www.congress.gov/bill/115th-congress/housebill/2430 (accessed 6.29.19).

Wang, Y., Witten, I.H., 1997. Inducing Model Trees for Continuous Classes, in: In Proc. of the 9th European Conf. on Machine Learning Poster Papers. pp. 128-137. 
Willing, R., Kim, I.Y., 2012. Quantifying the competing relationship between durability and kinematics of total knee replacements using multiobjective design optimization and validated computational models. J. Biomech. 45, 141-147. https://doi.org/10.1016/j.jbiomech.2011.09.008

Willing, R., Kim, I.Y., 2011. Design optimization of a total knee replacement for improved constraint and flexion kinematics. J. Biomech. 44, 1014-1020. https://doi.org/10.1016/j.jbiomech.2011.02.009

Zavatsky, A.B., 1997. A kinematic-freedom analysis of a flexed-knee-stance testing rig. J. Biomech. 30, 277-280. https://doi.org/10.1016/S0021-9290(96)00142-X

Zienkiewicz, O.C., Taylor, R.L., Zhu, J., 2010. The finite element method. Vol. 1: Its basis and fundamentals, 6. ed., repr. ed. Elsevier Butterworth-Heinemann, Amsterdam. 


\section{APPENDIX A}

\section{COMBINED SET - TWO SAMPLE T-TEST FACTOR SENSITIVITIES}

The following charts were produced using the dataset most likely to be used in a design scenario, the combination set with linear predictors and a sample rate of 10 simulations per parameter. Two-sample t-tests with $\alpha=0.05$ were performed for every factor and response combination using the same high and low factor blocks created during factoreffect screening (Figure 12). The appendix is split into functional group subsections, where each subsection contains a chart ranking factors by significance. If the factor was significant for a higher proportion of the functional group's output variables, the rank increased. Additionally, lookup tables are provided to search for significant factors. The first table in each subsection will be referenced by factor name, while the second will be referenced by response variable name. 


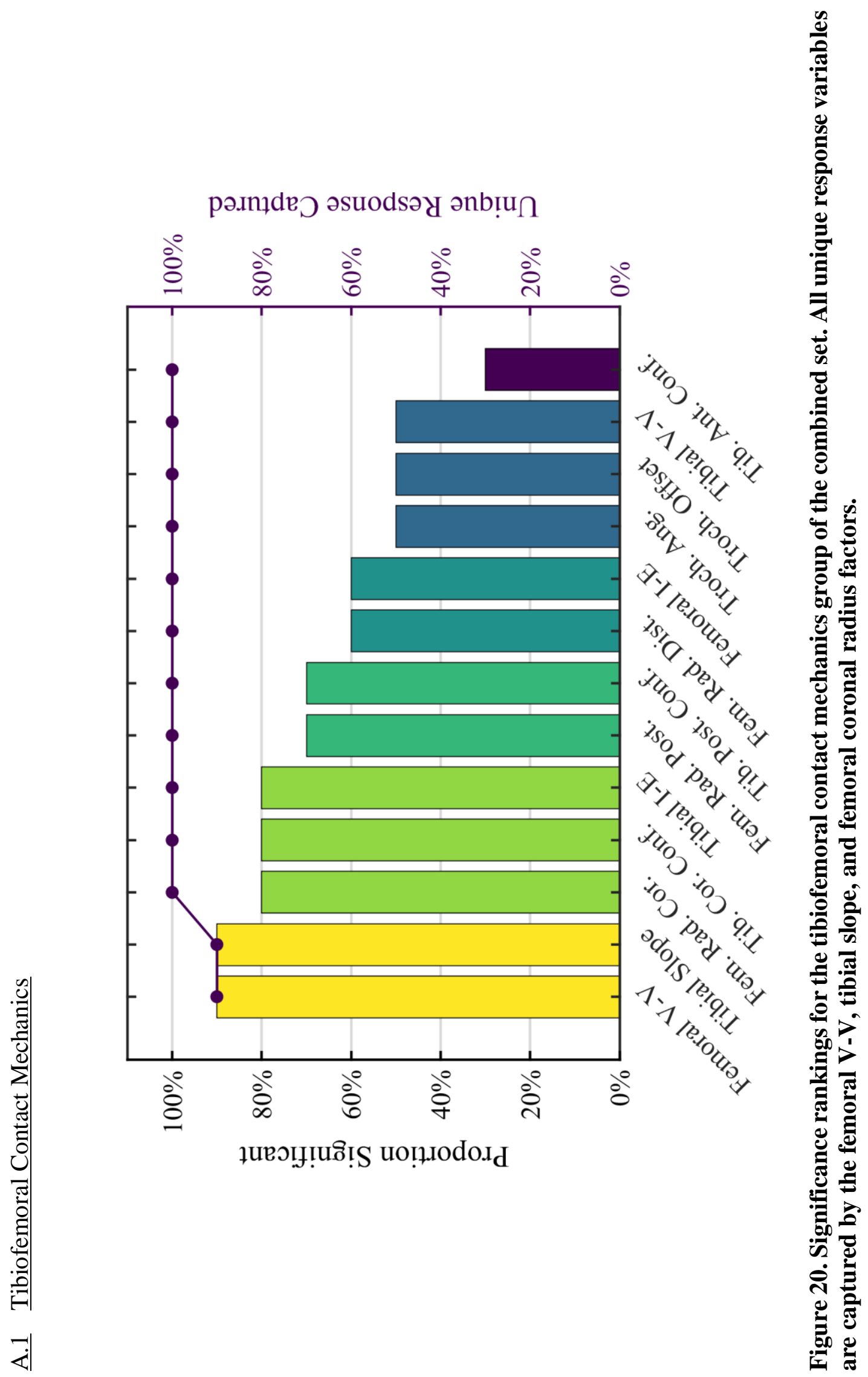




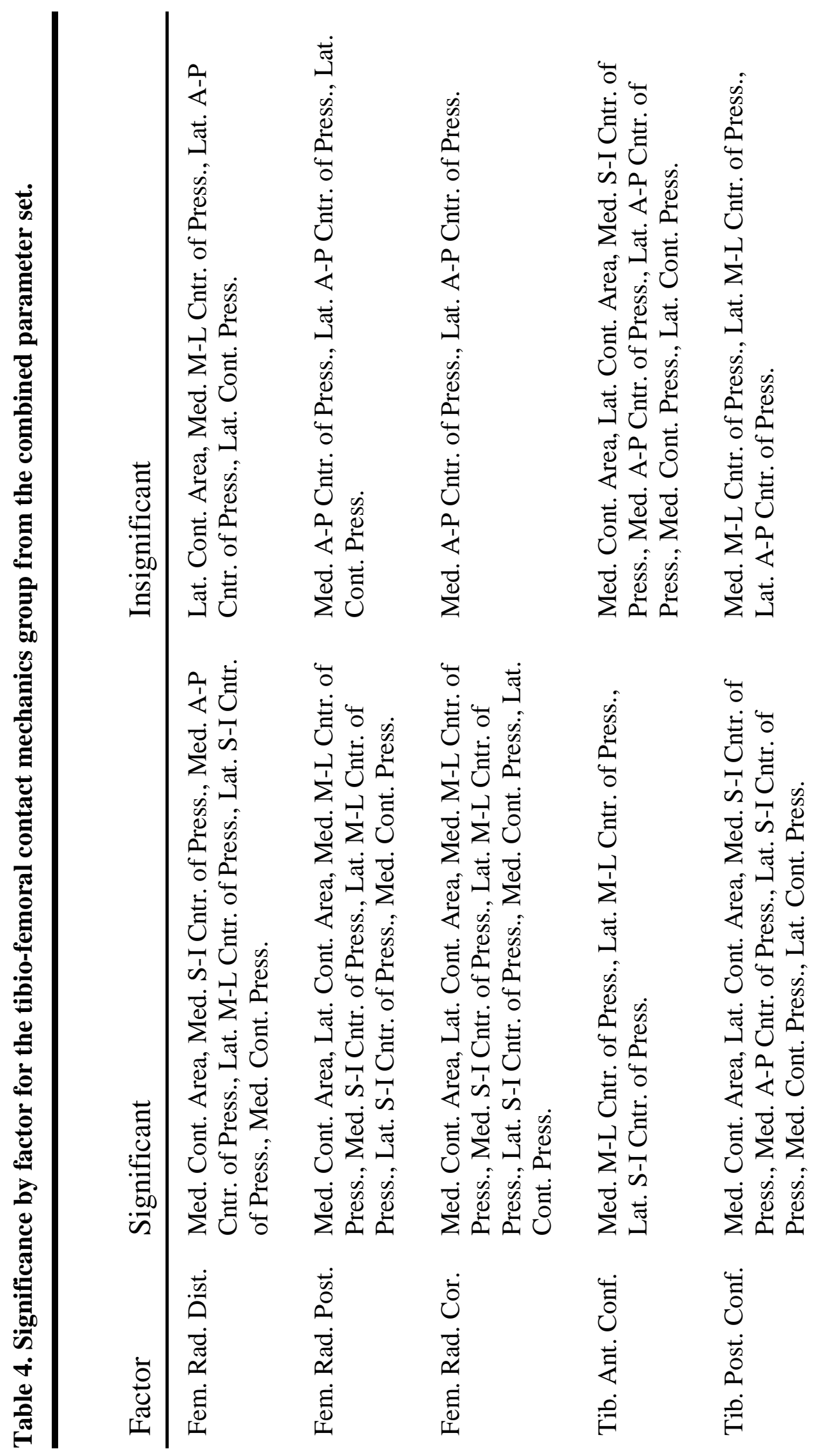




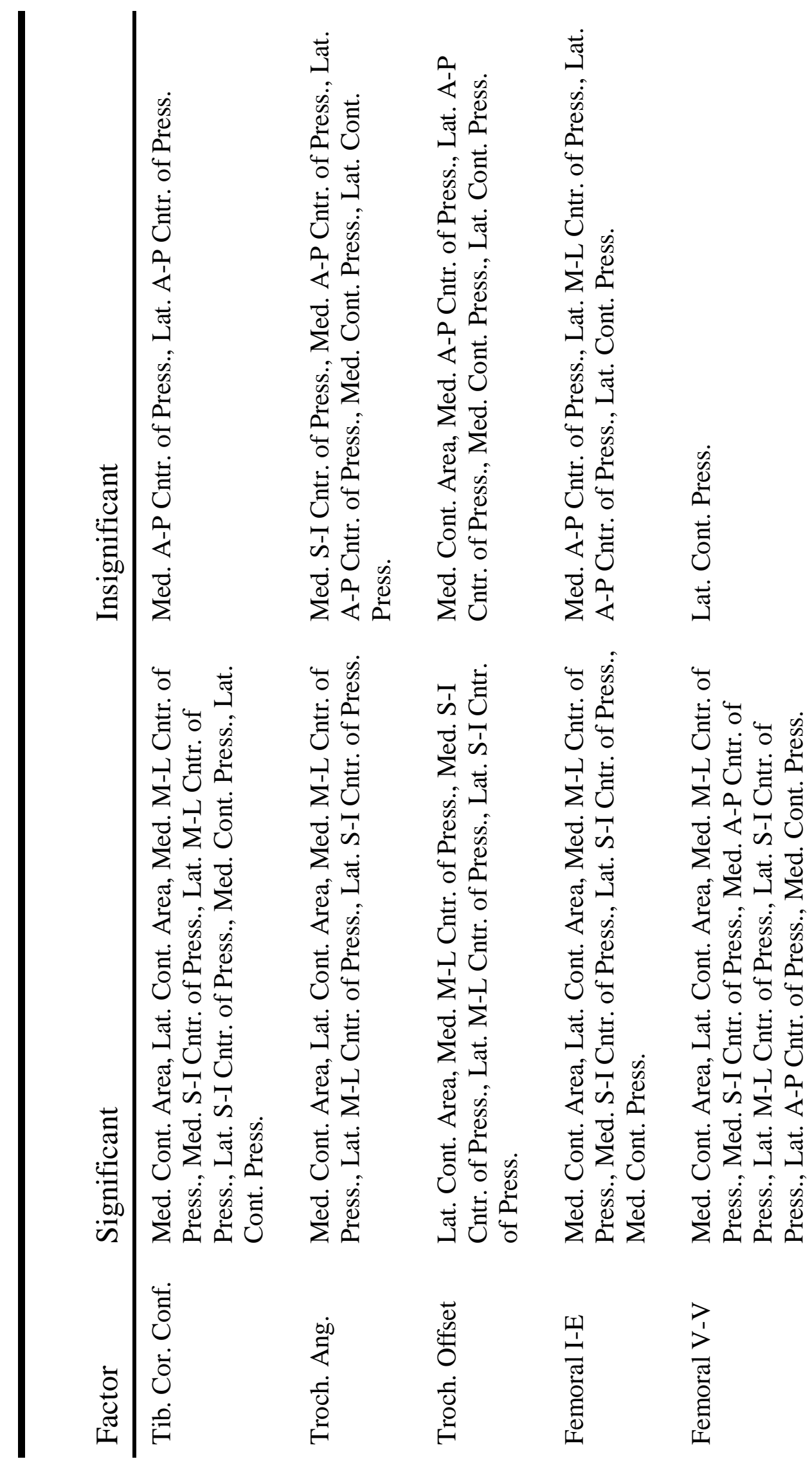




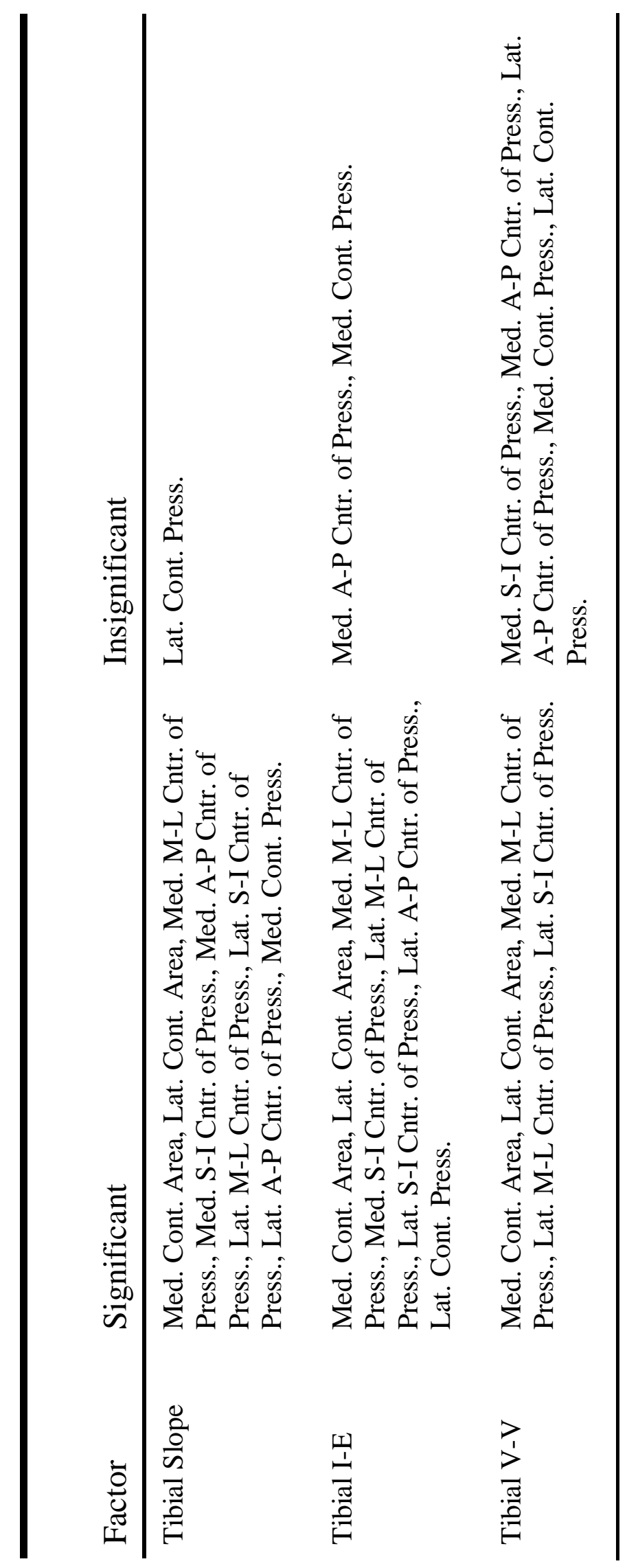



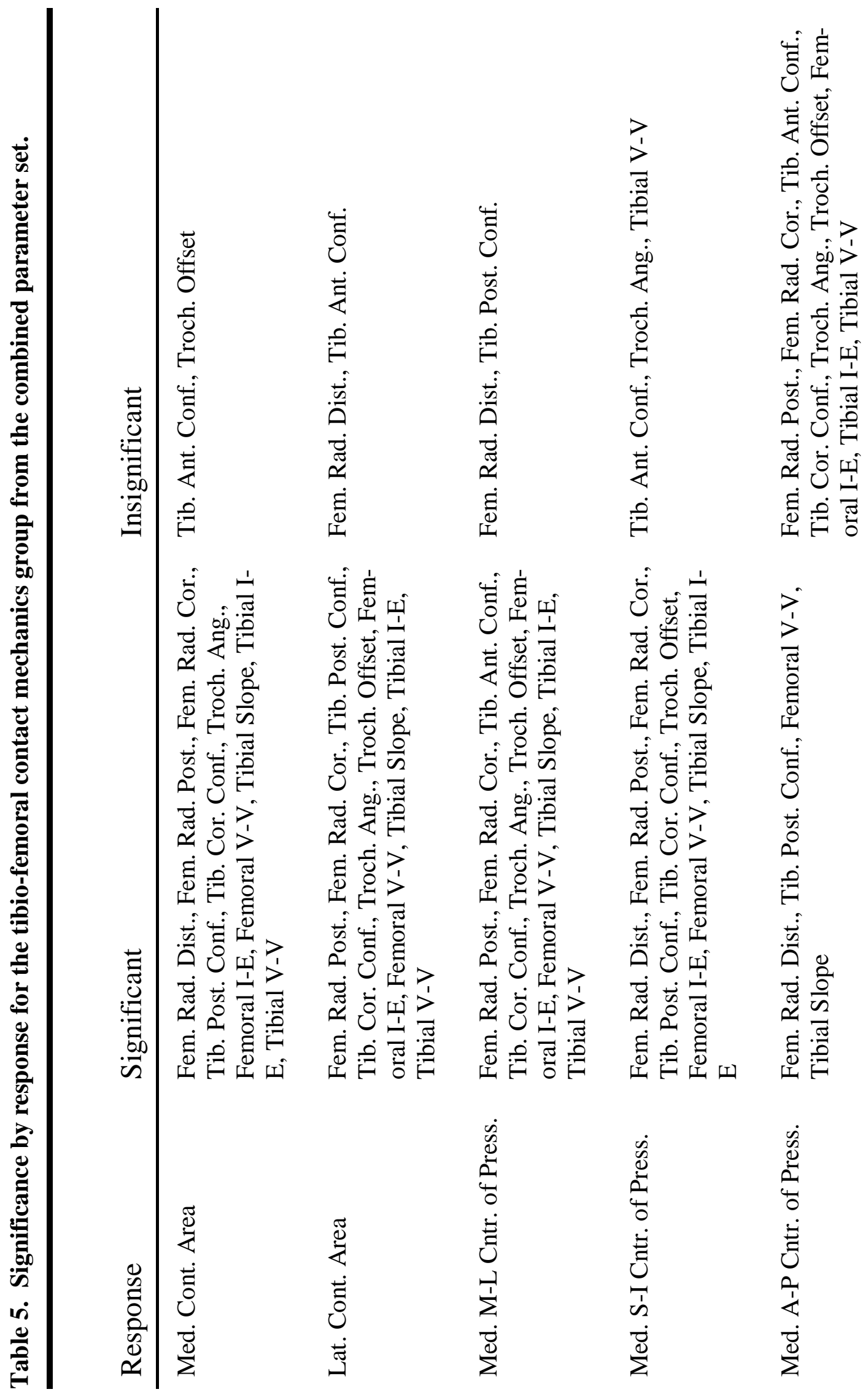


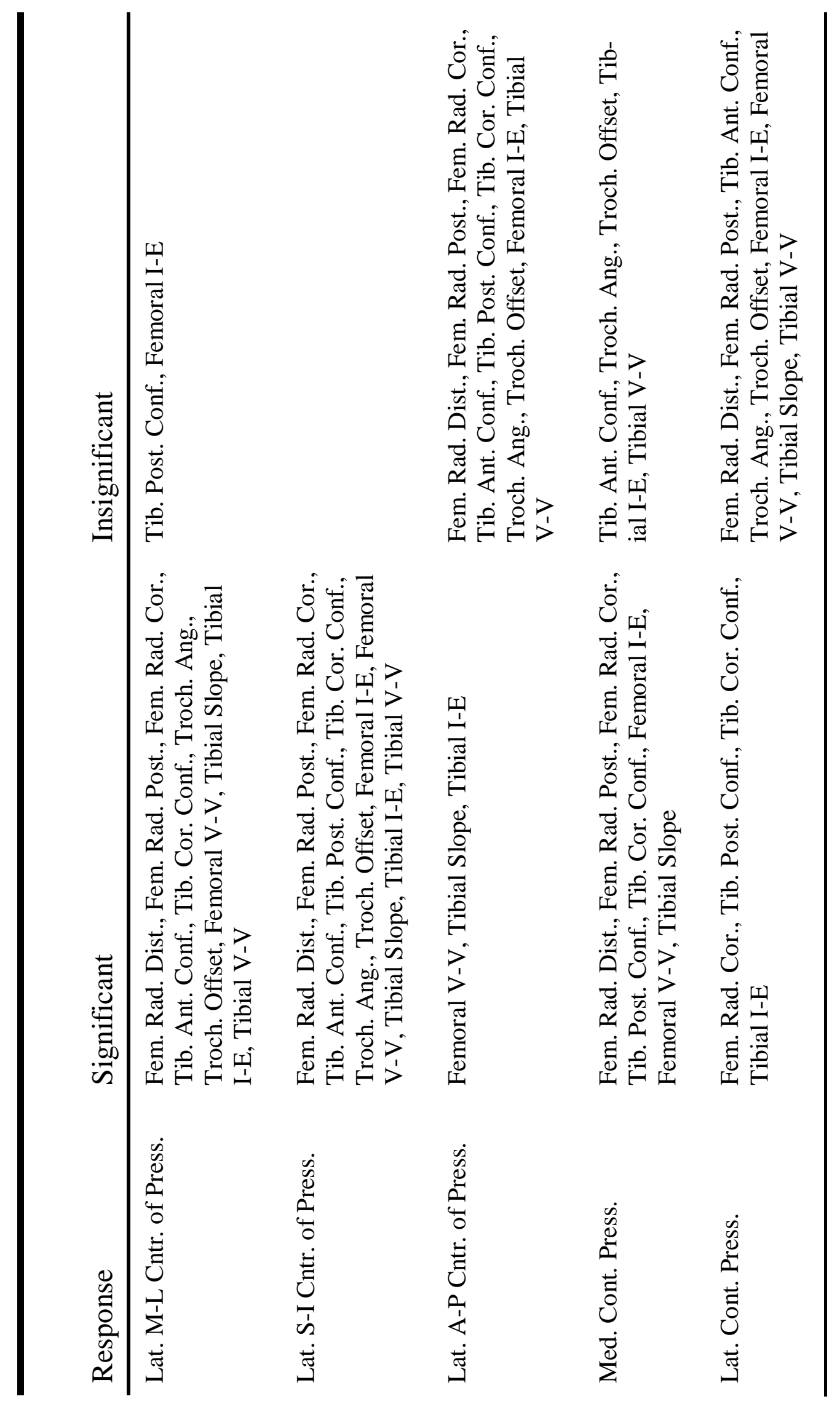




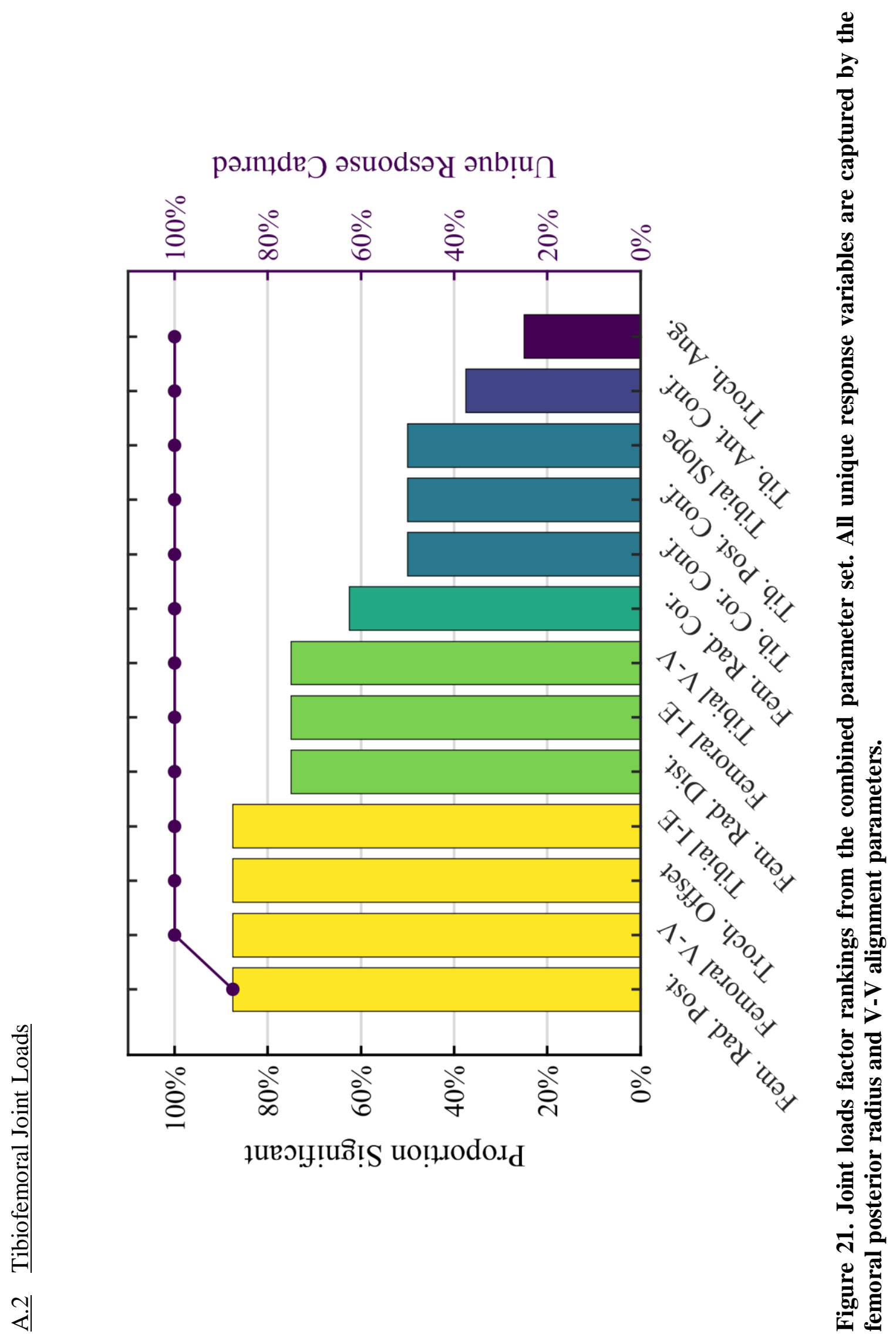




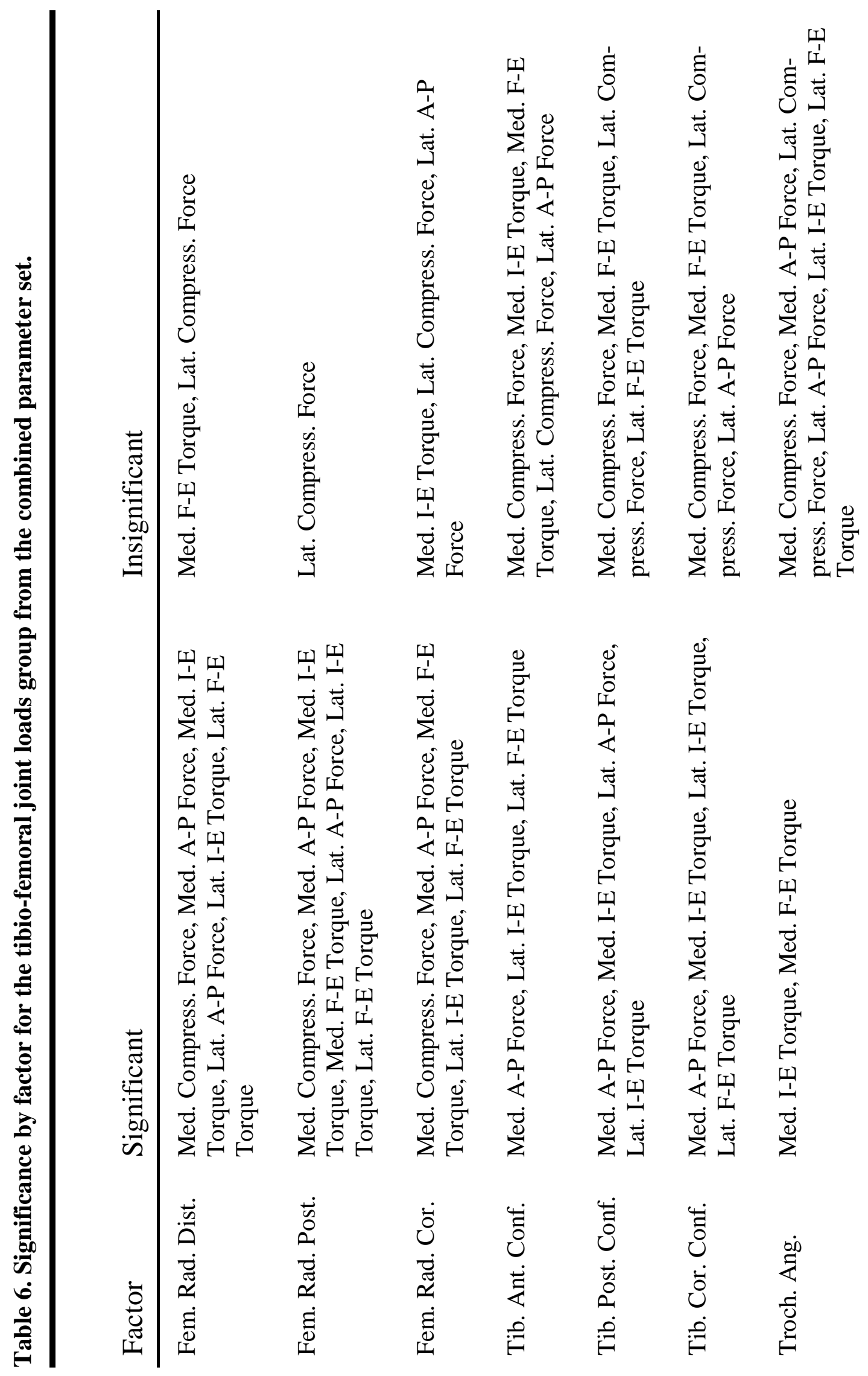




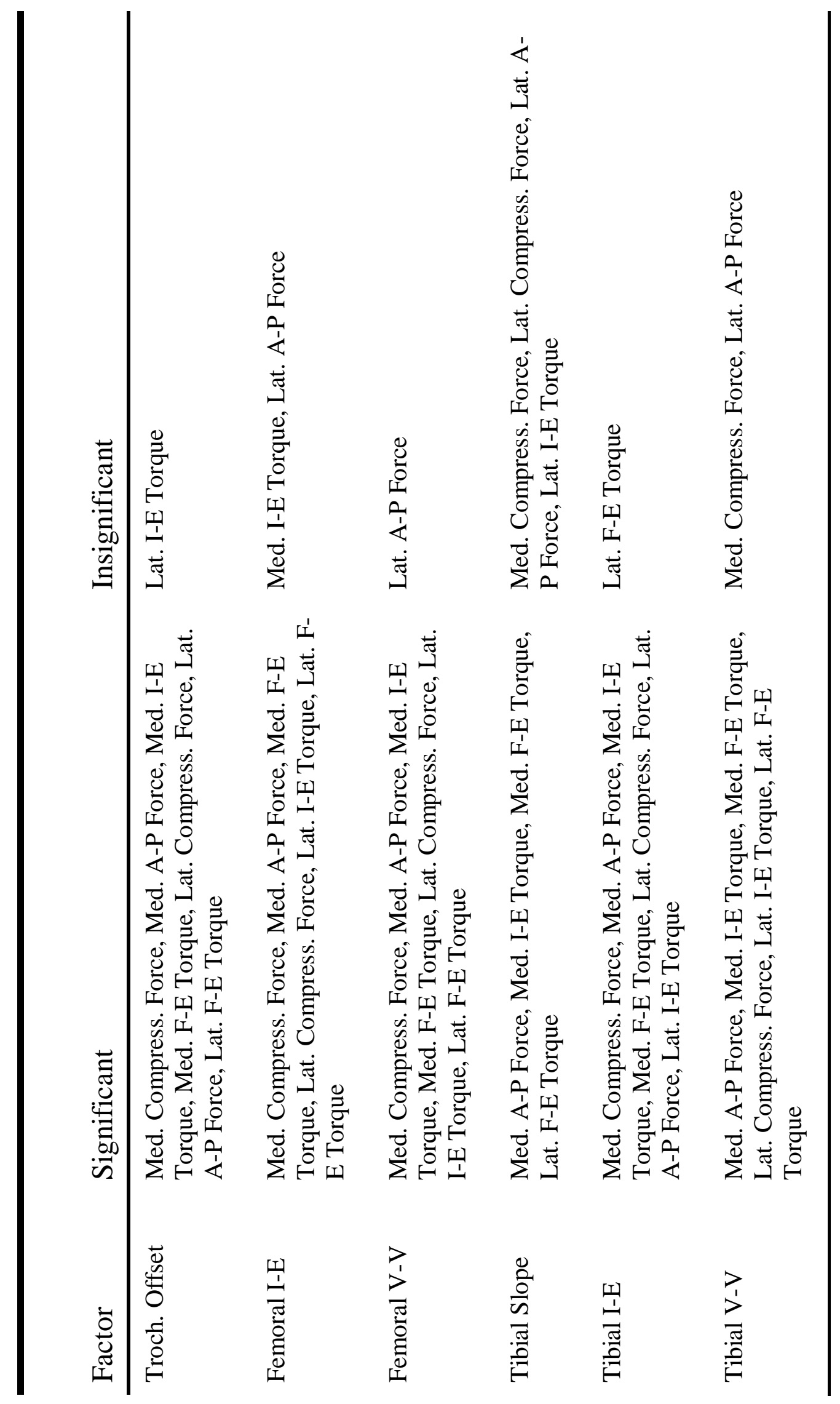




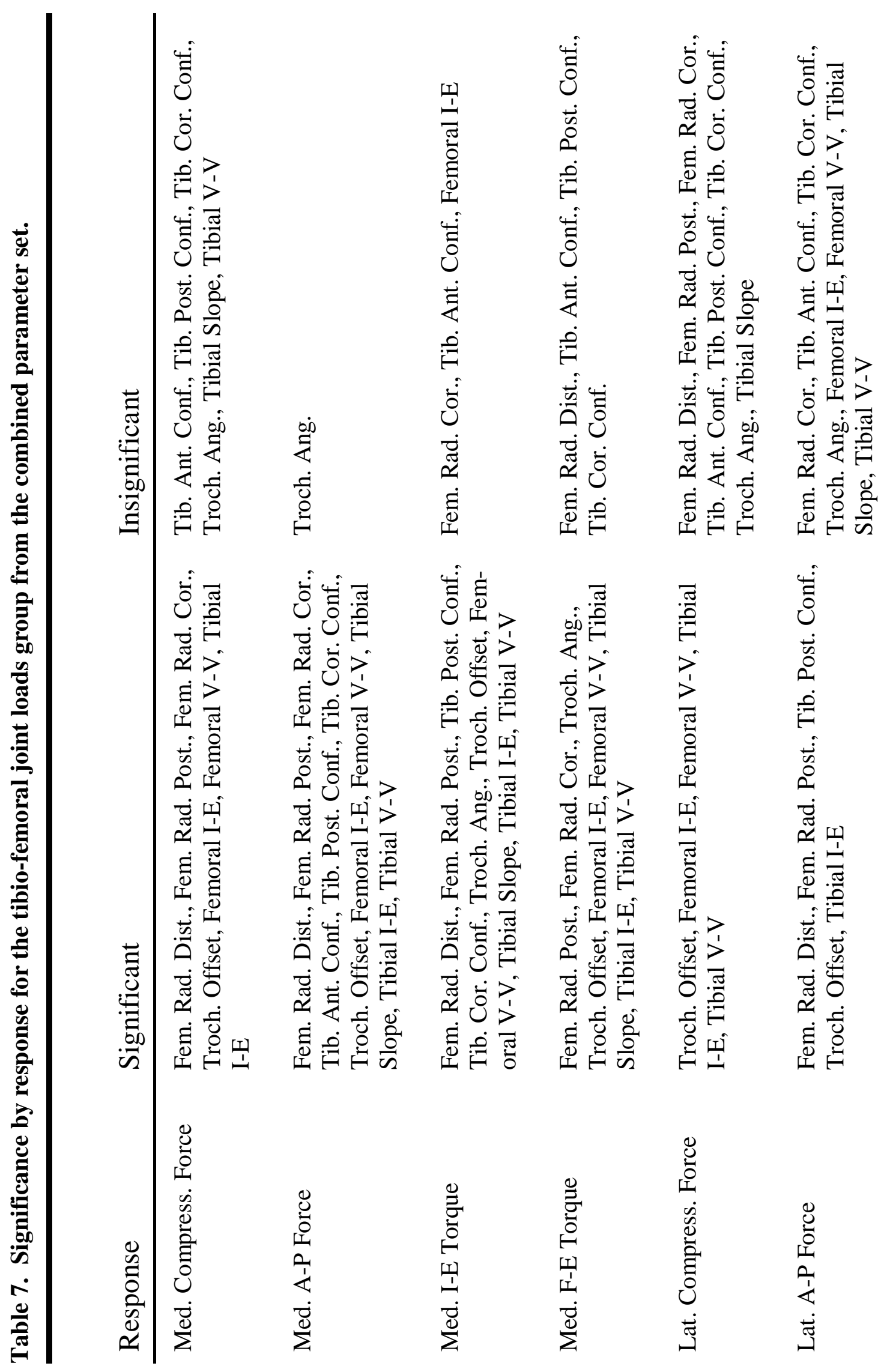




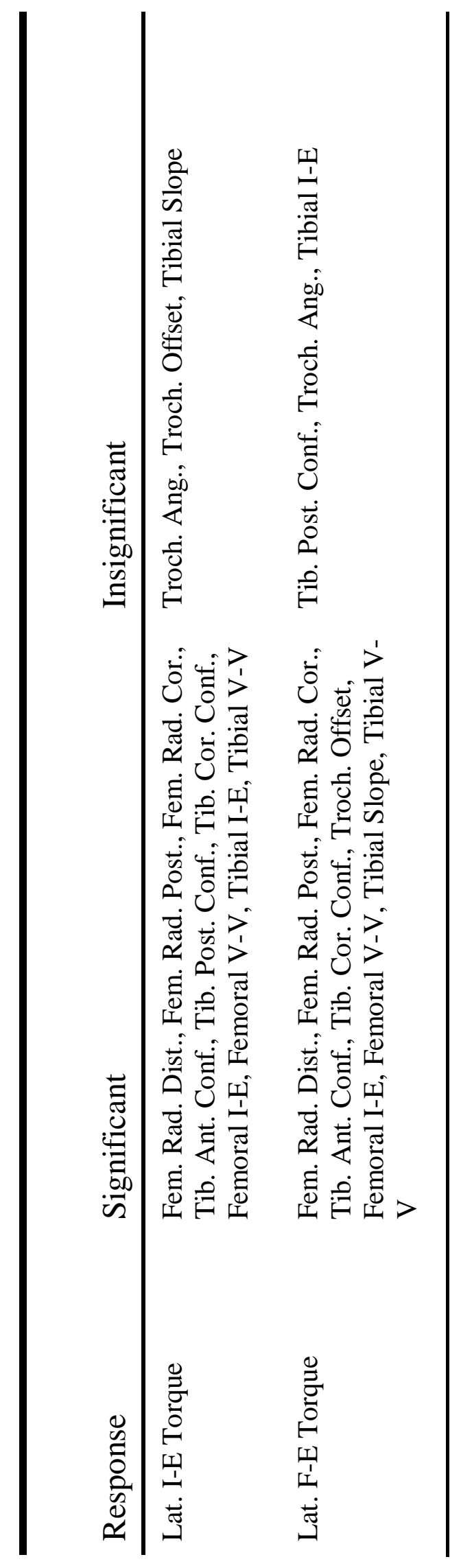




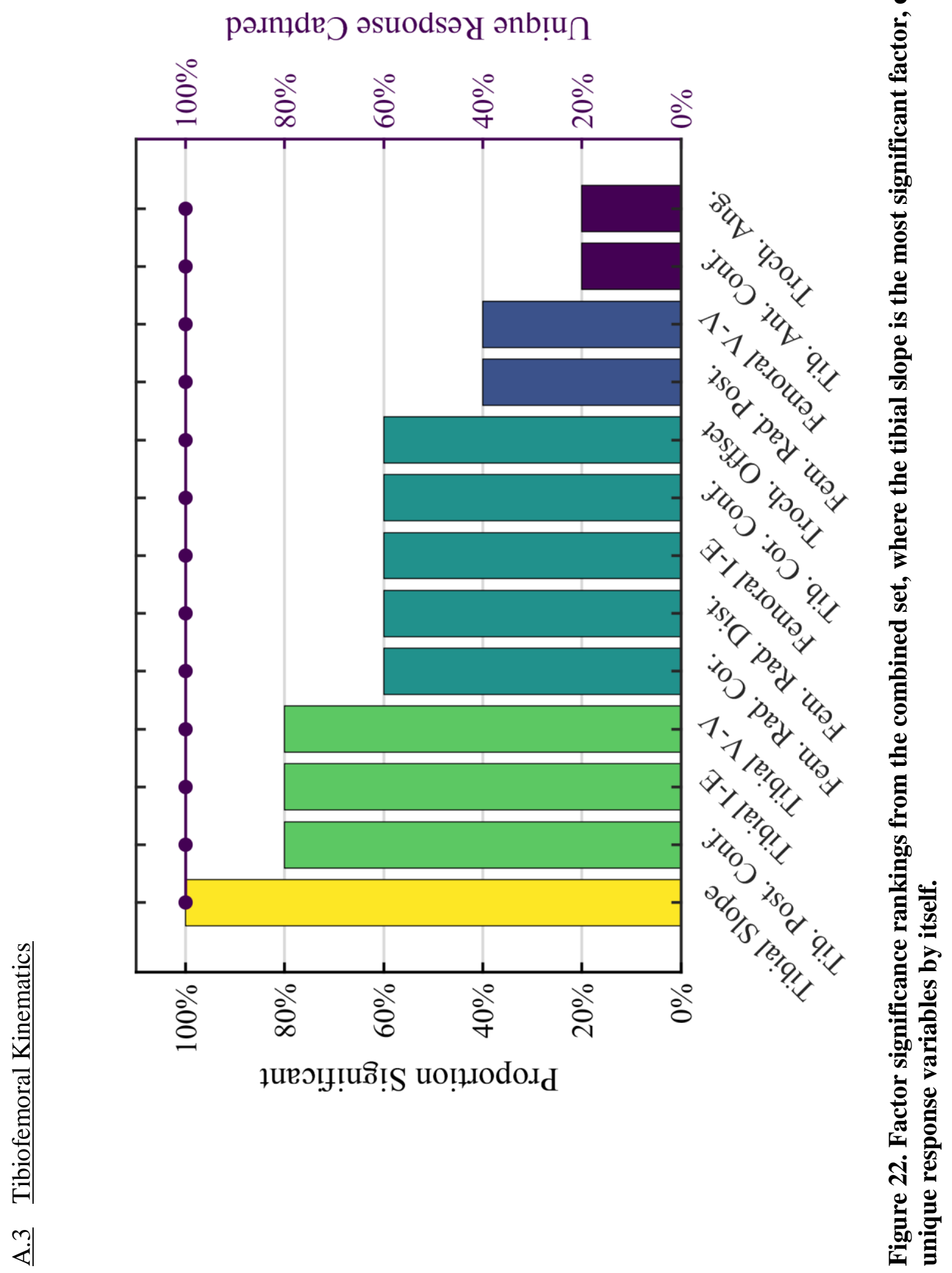




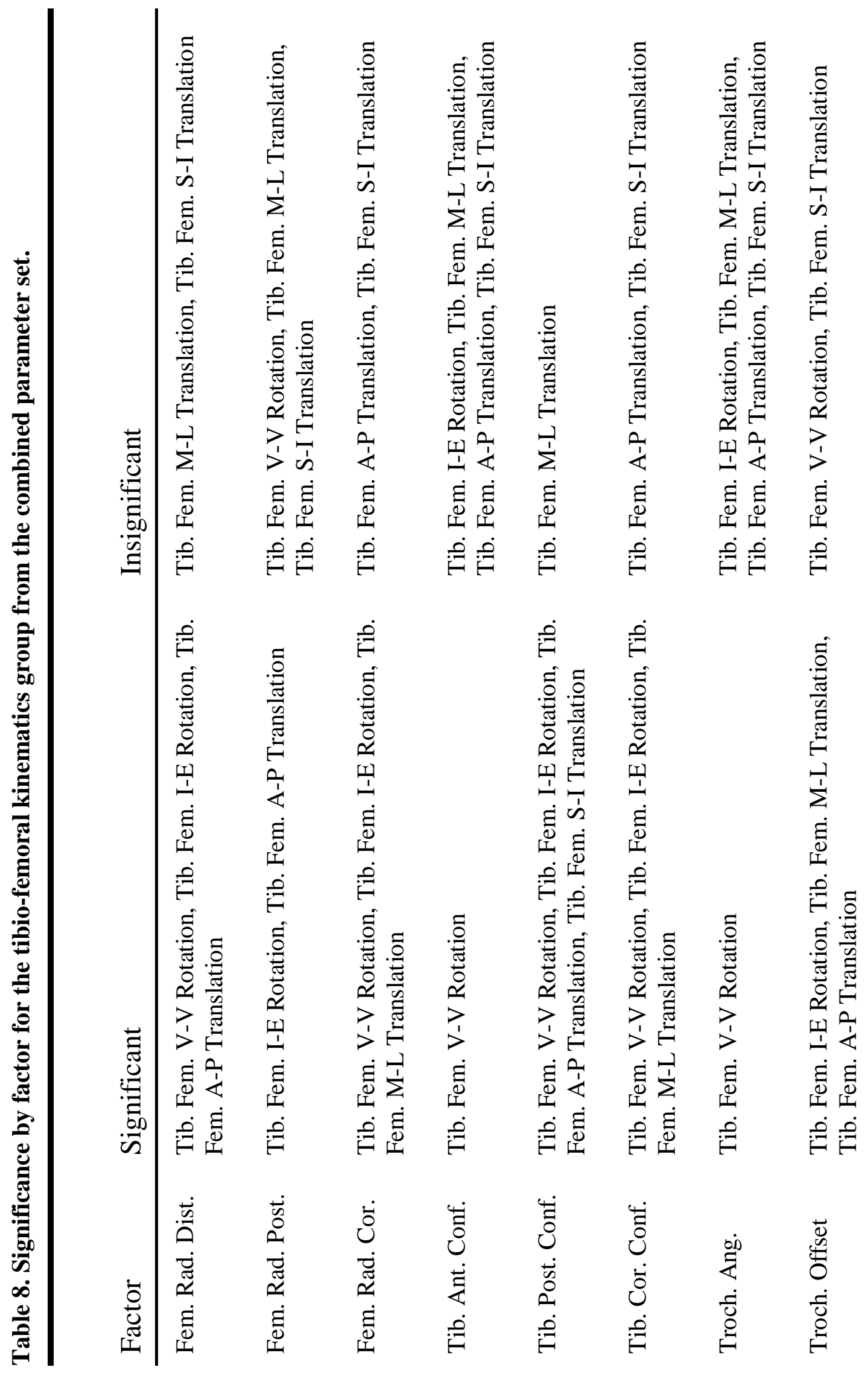




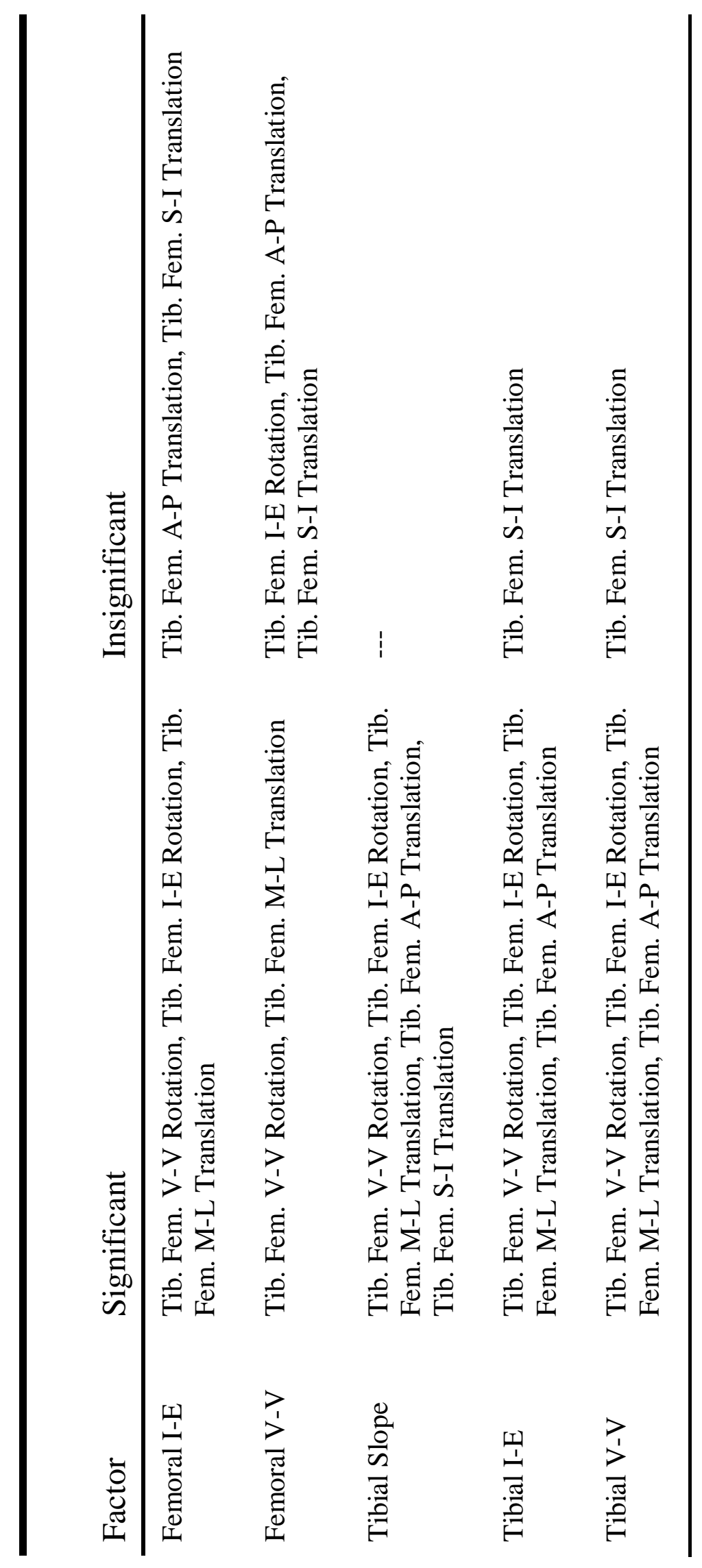




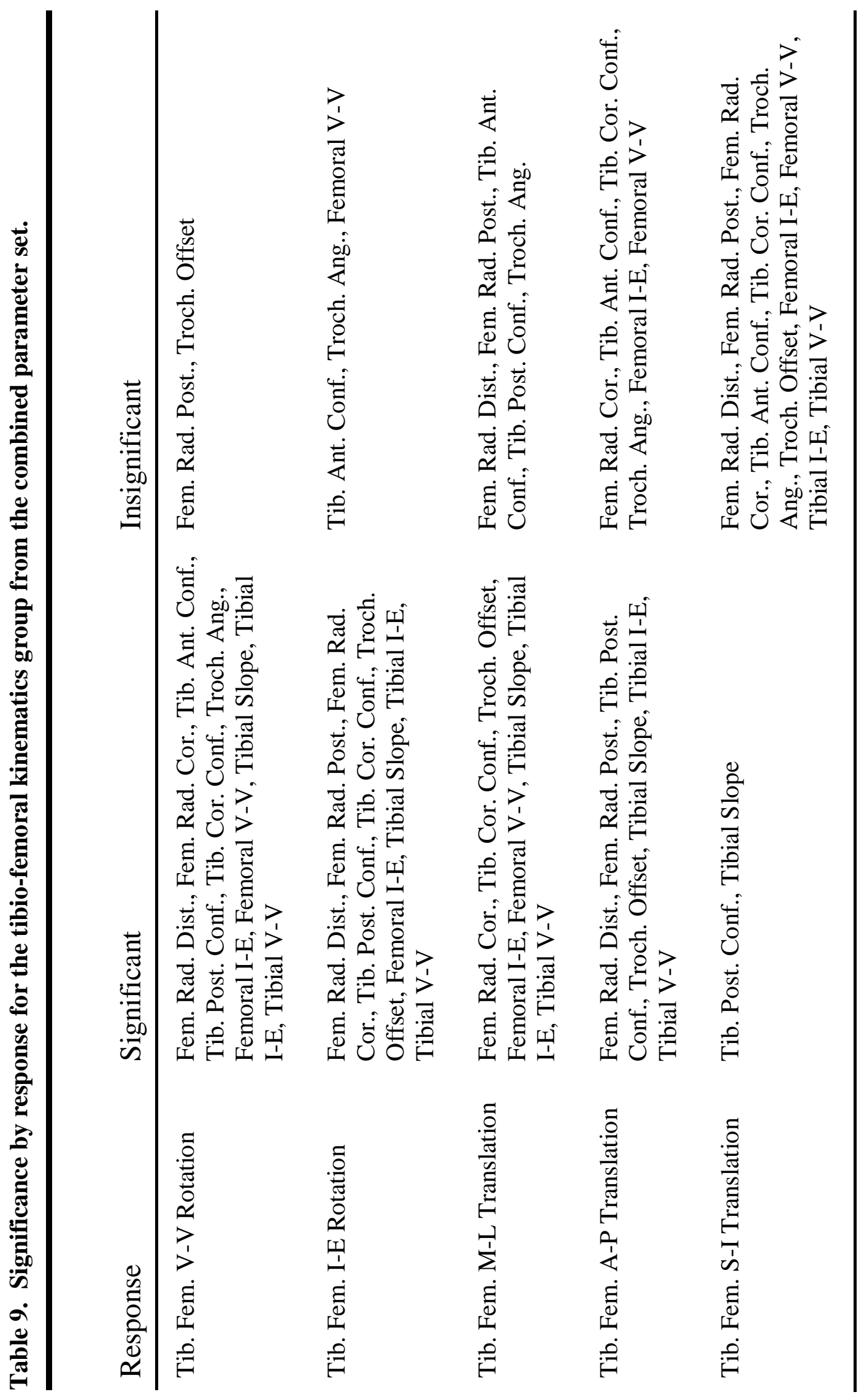




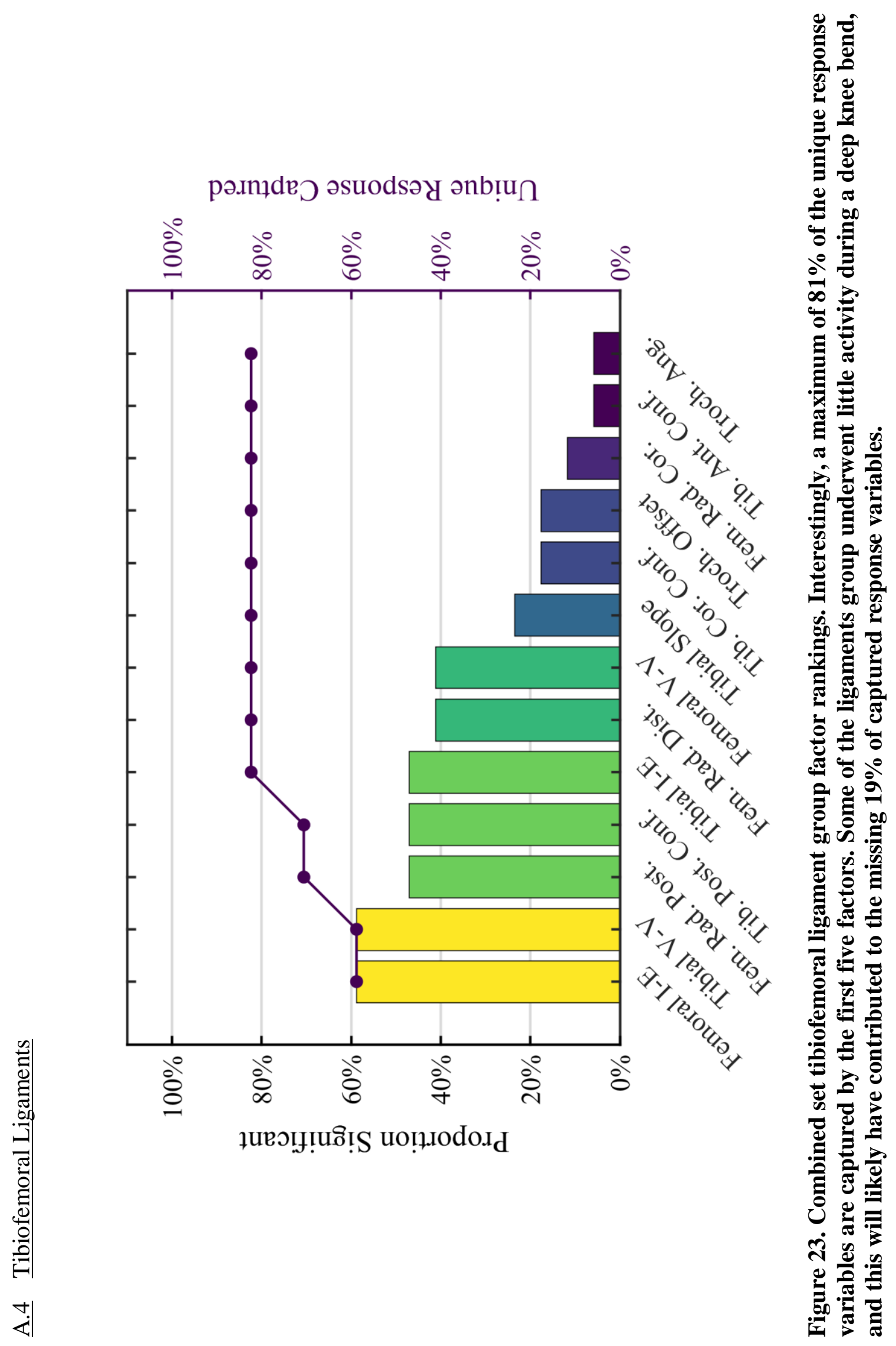




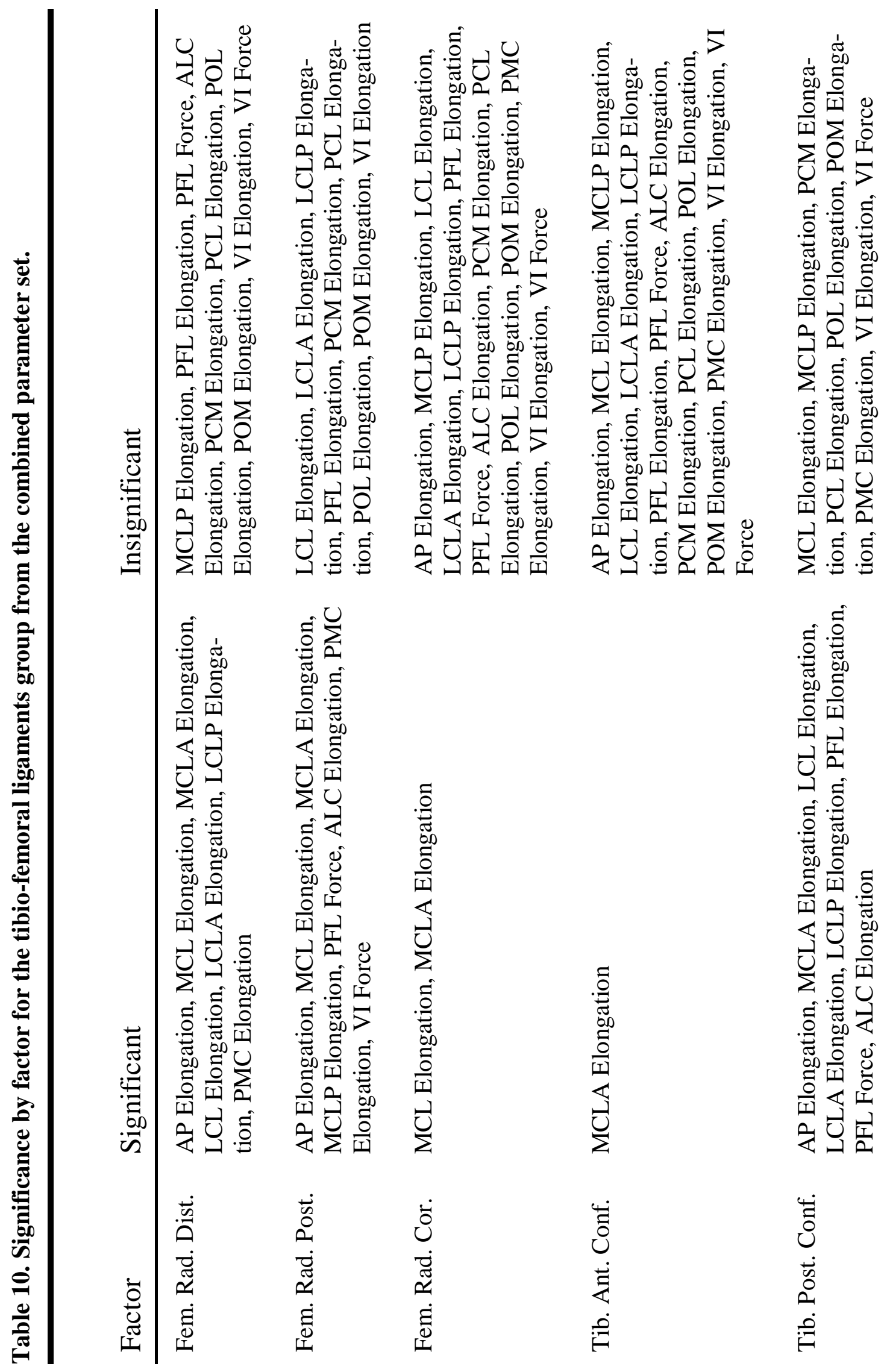




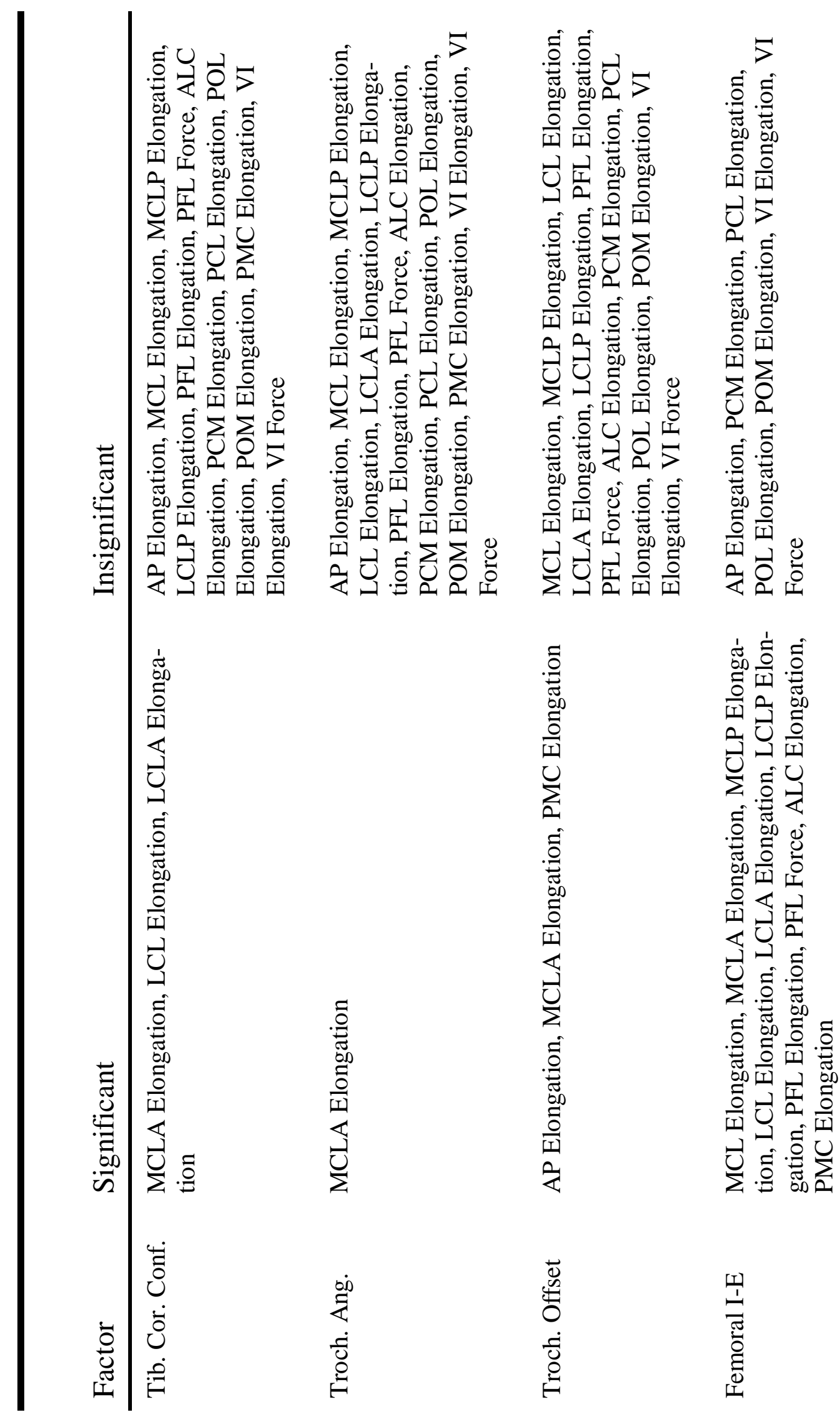




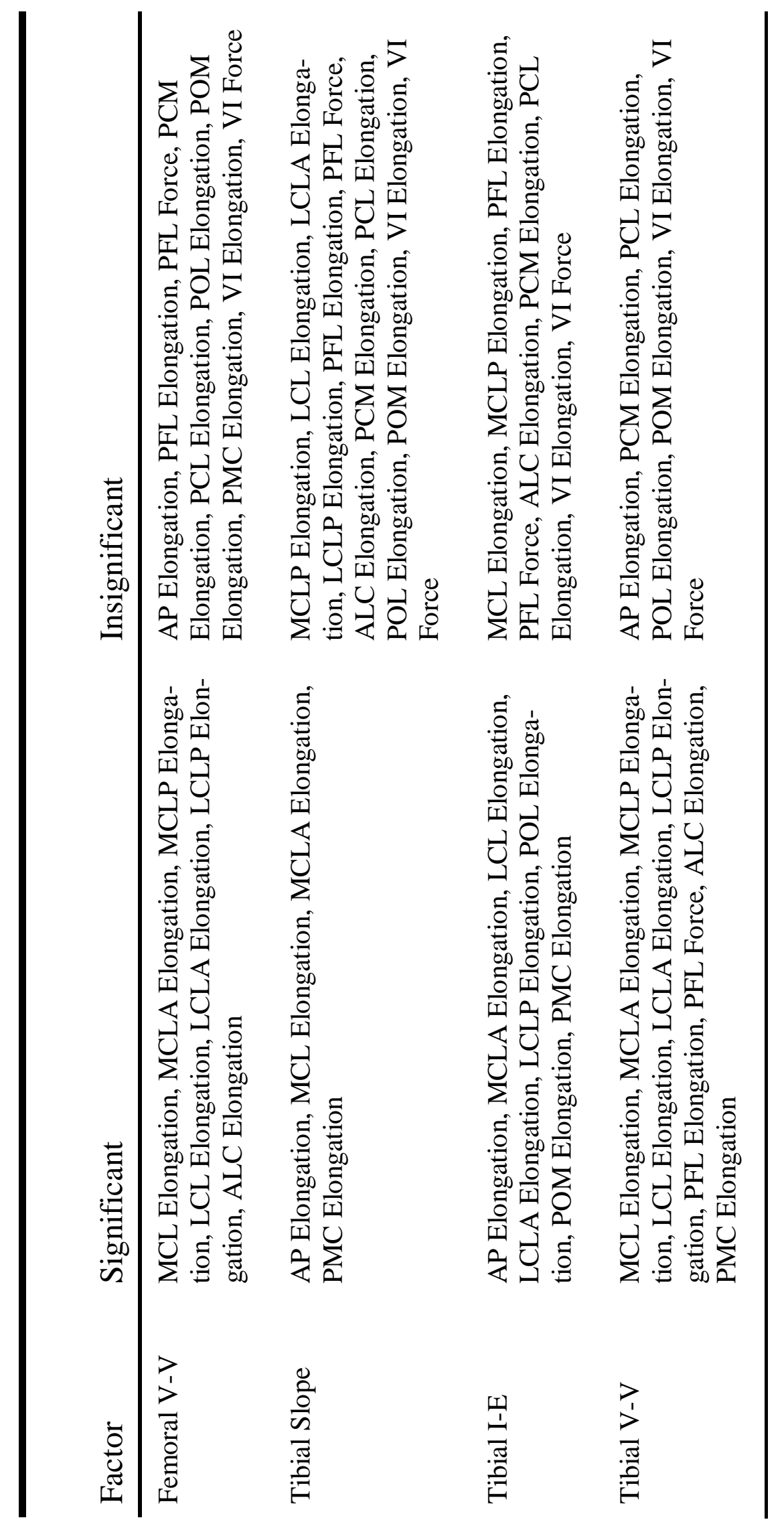




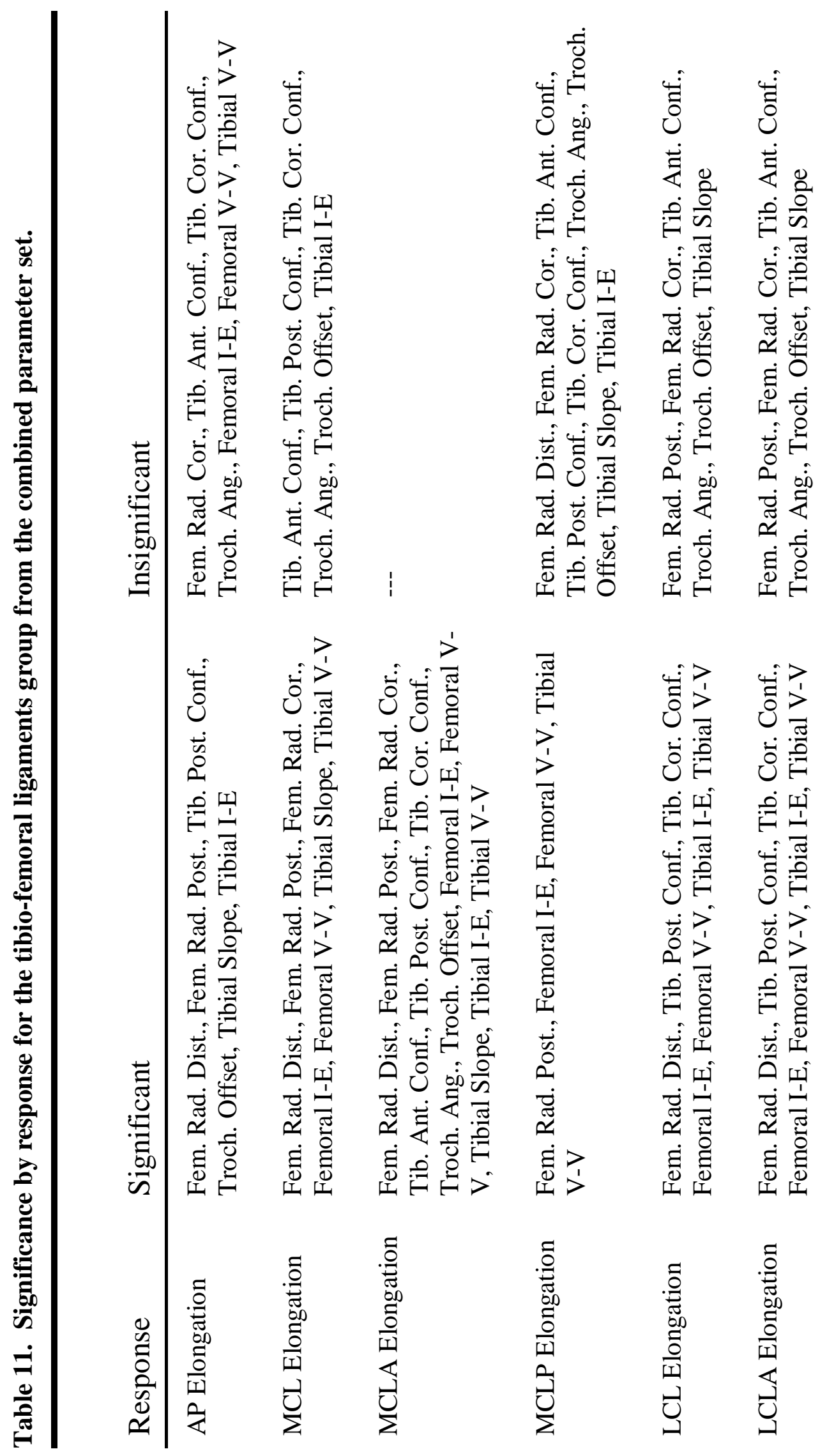




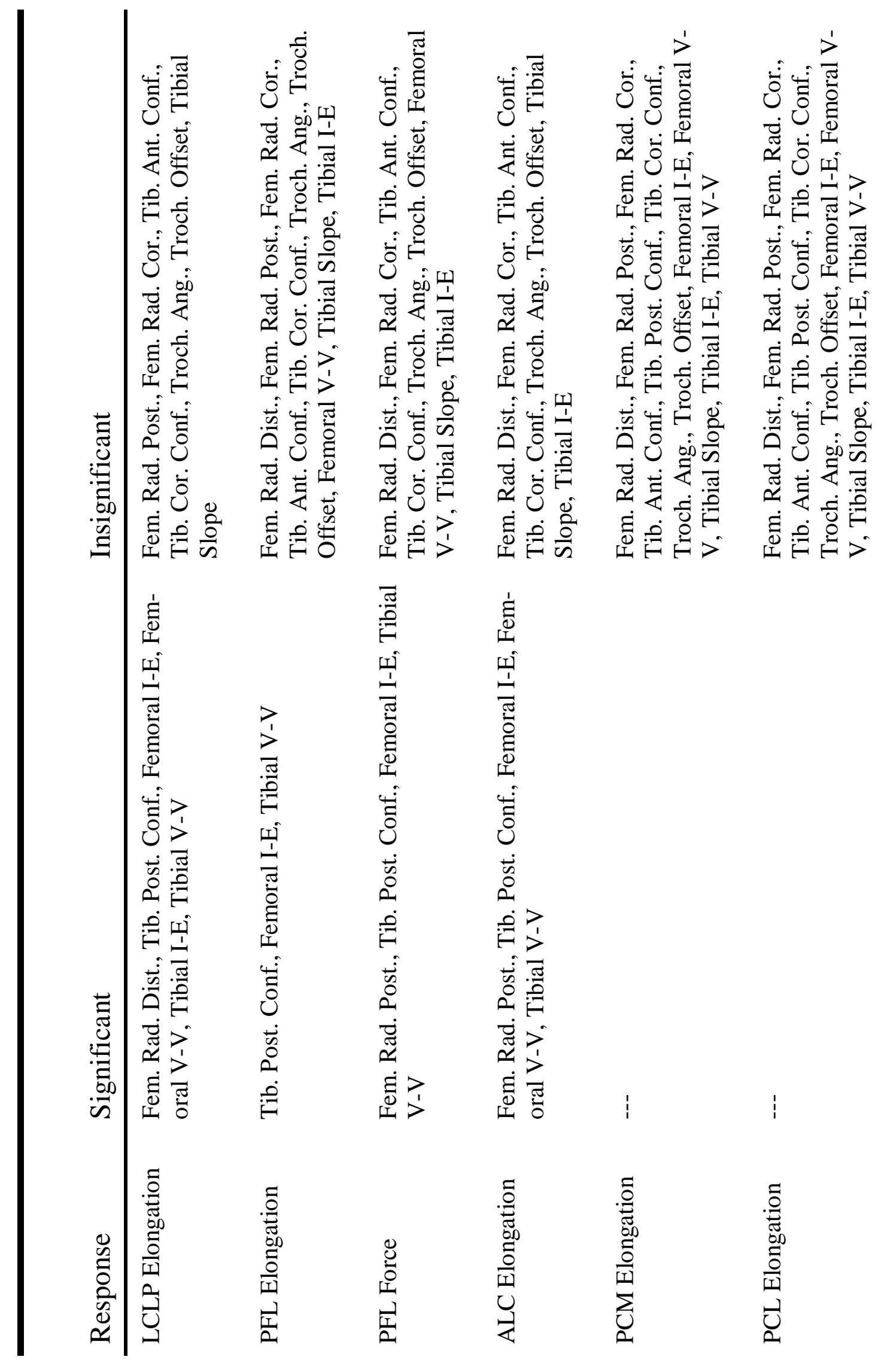




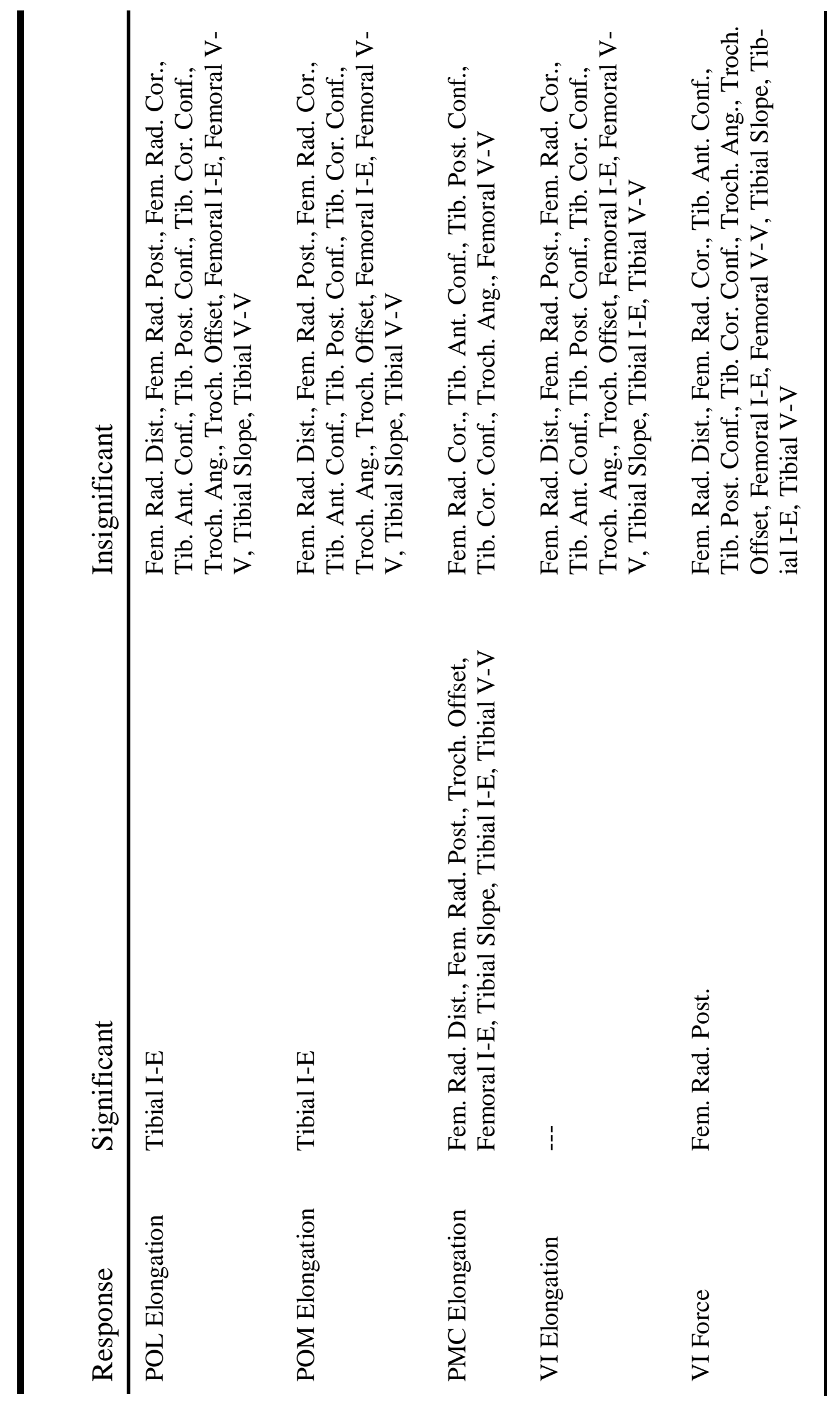




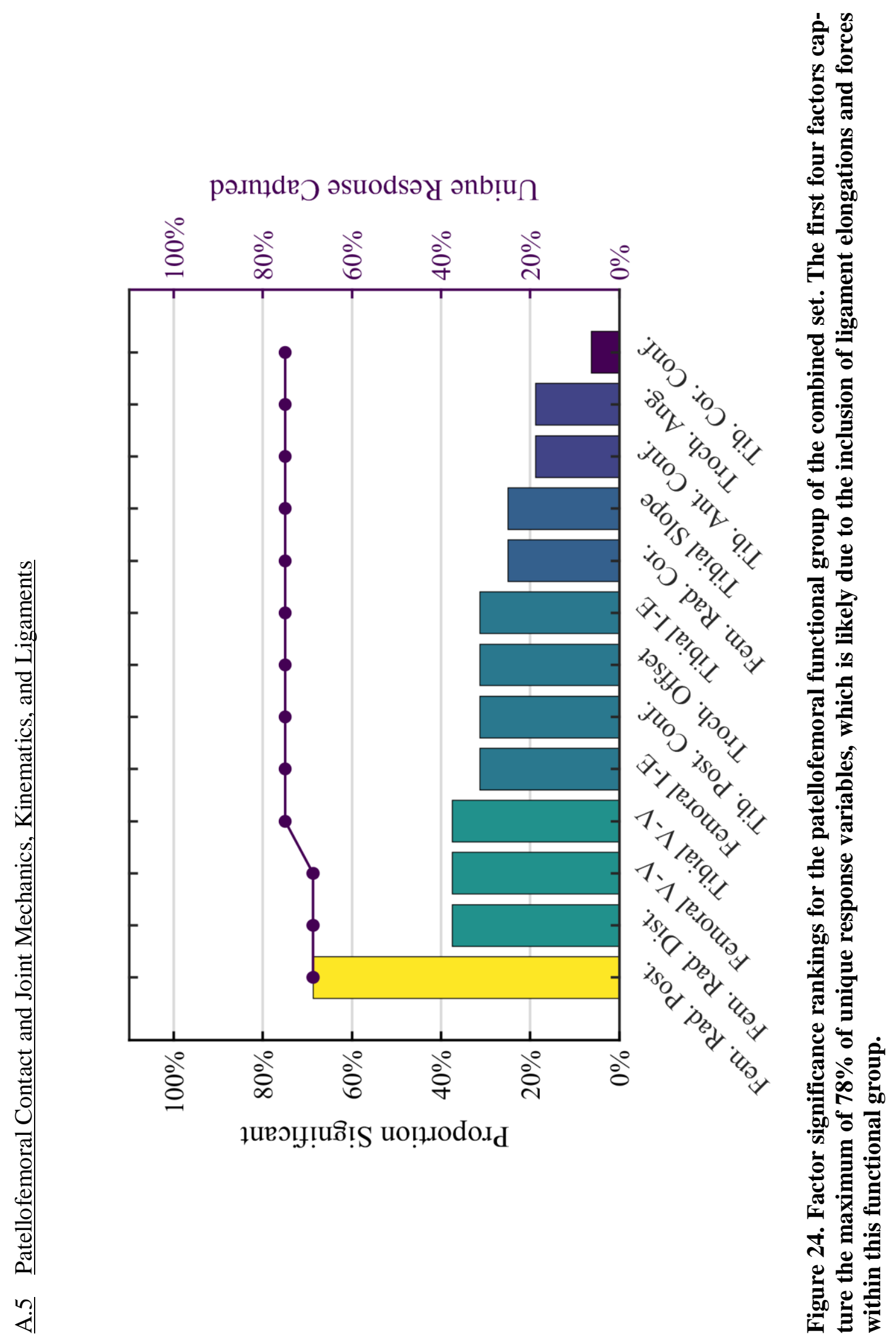




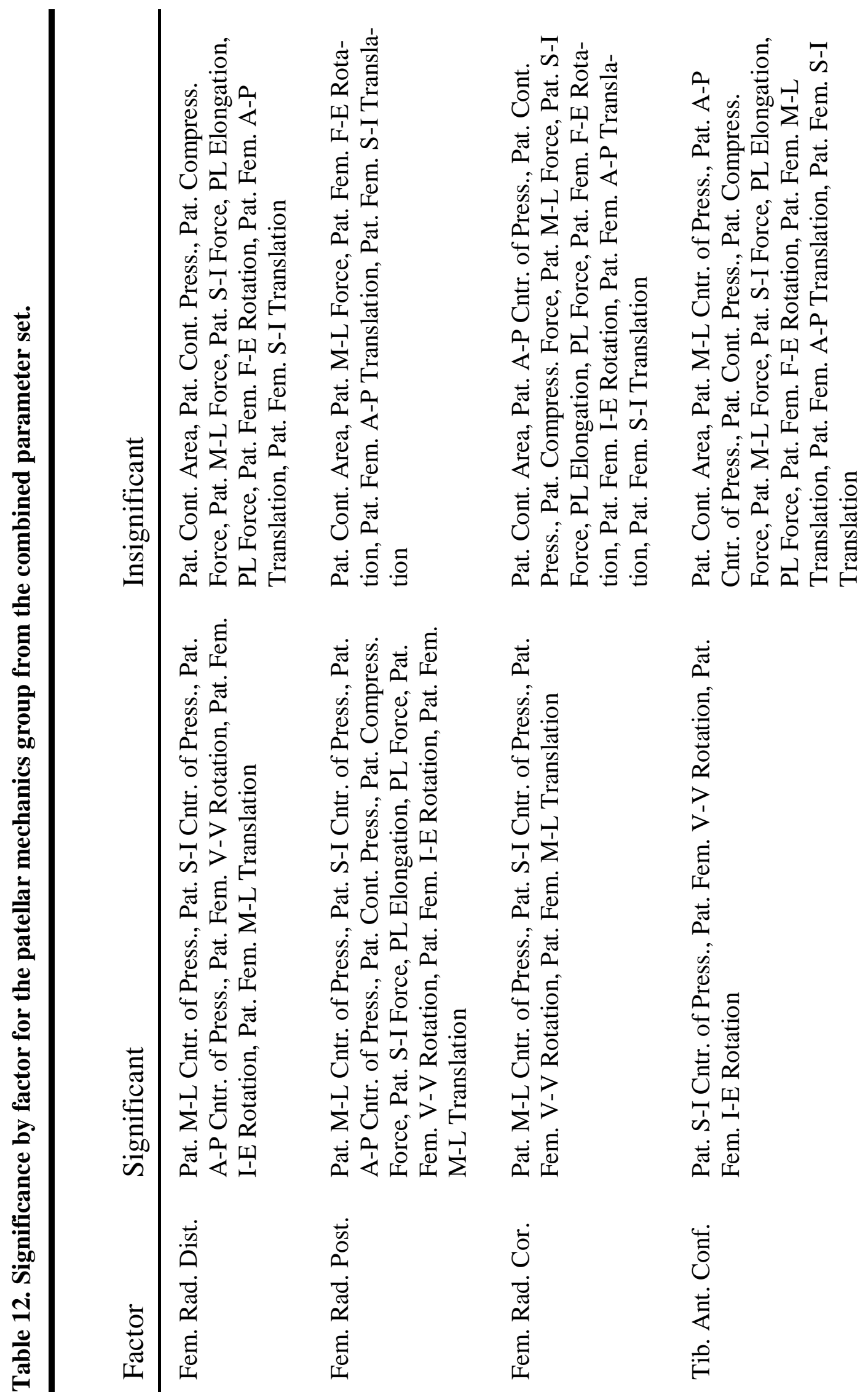




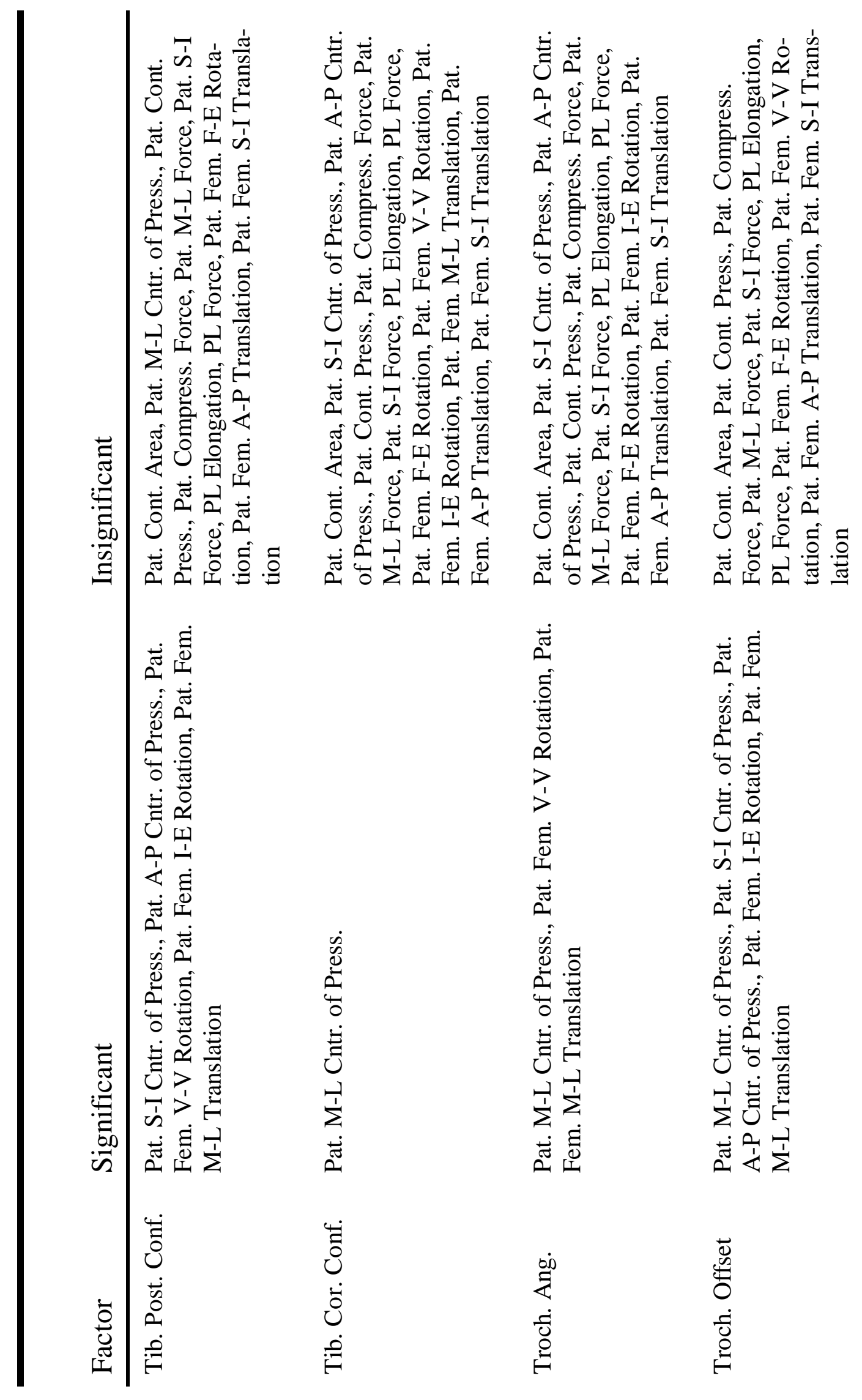




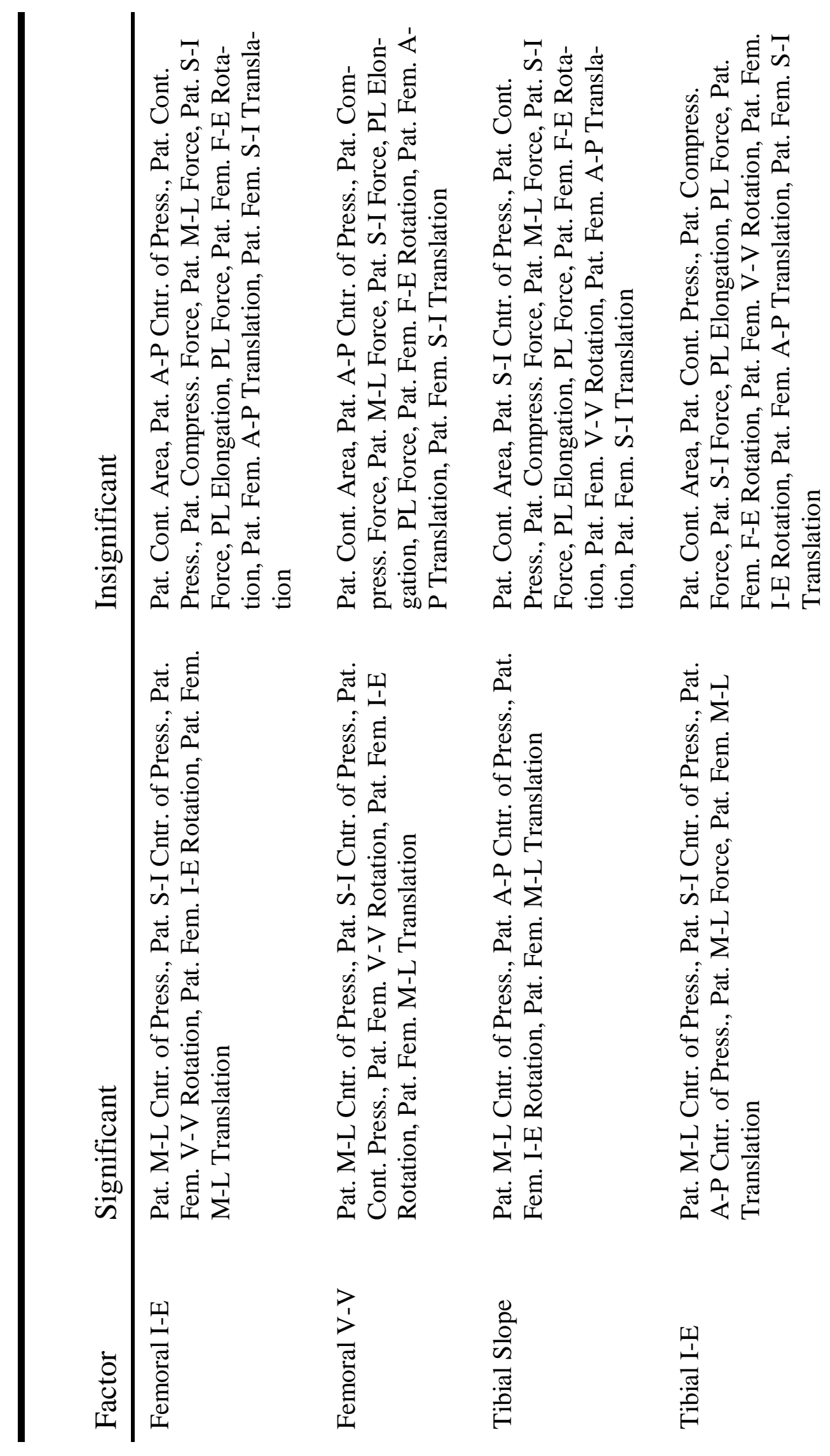




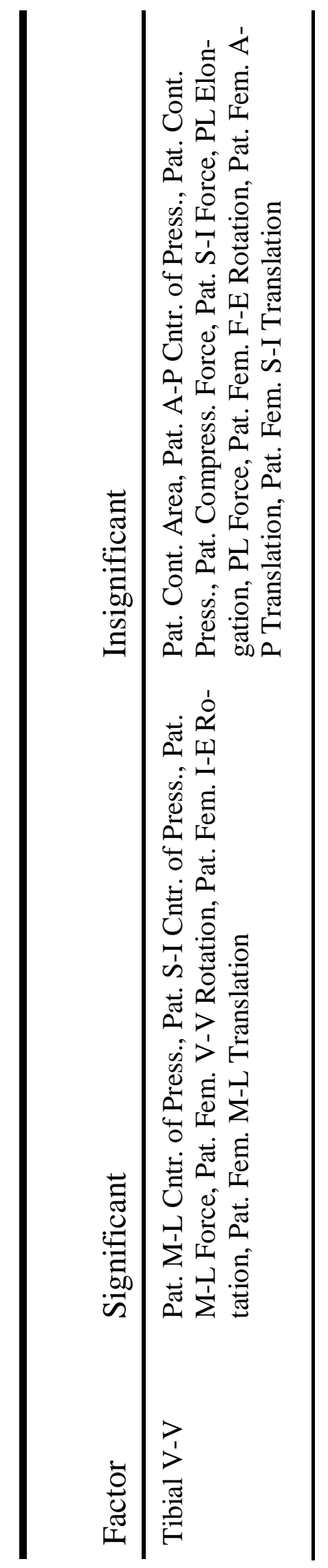




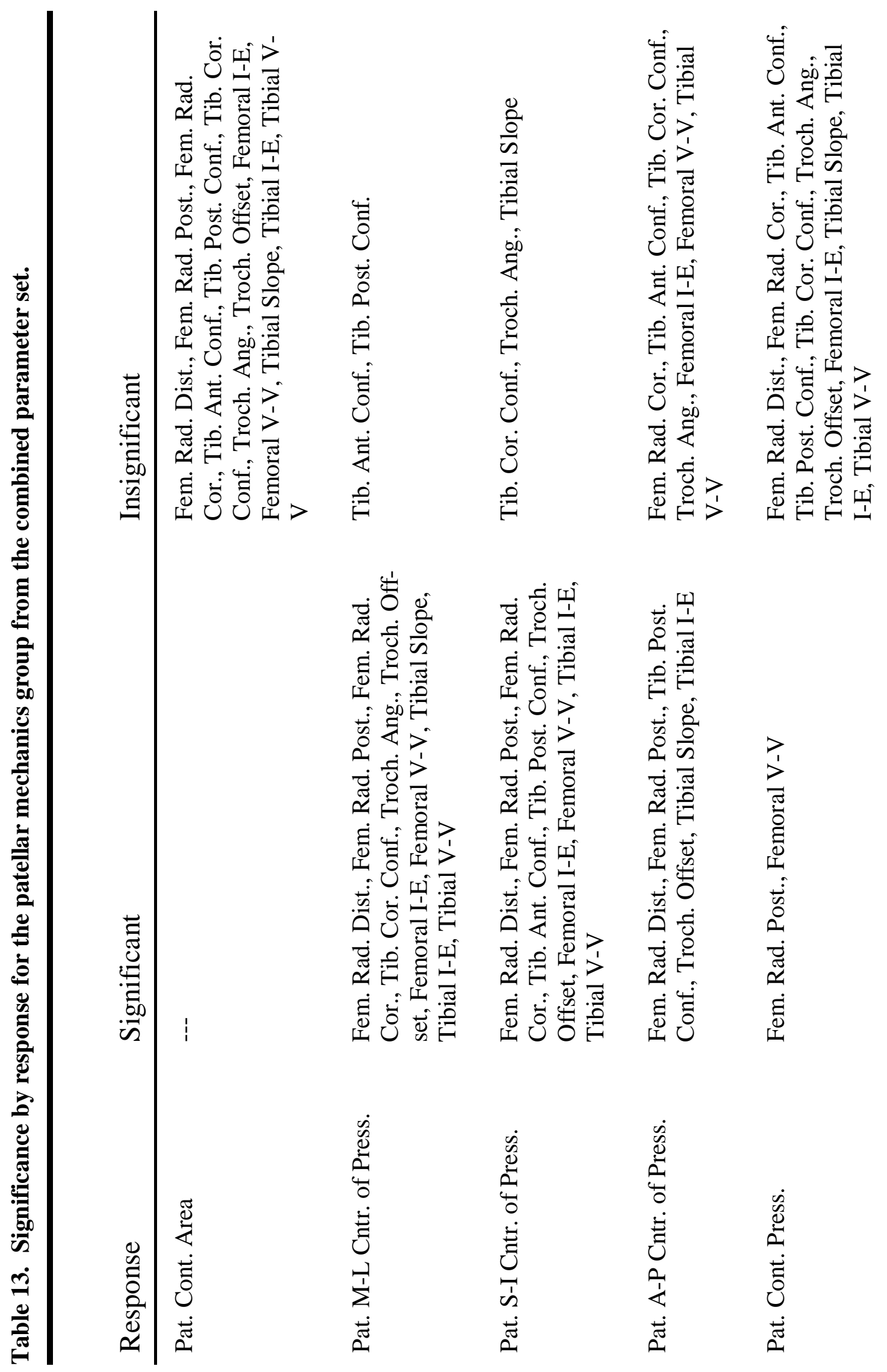




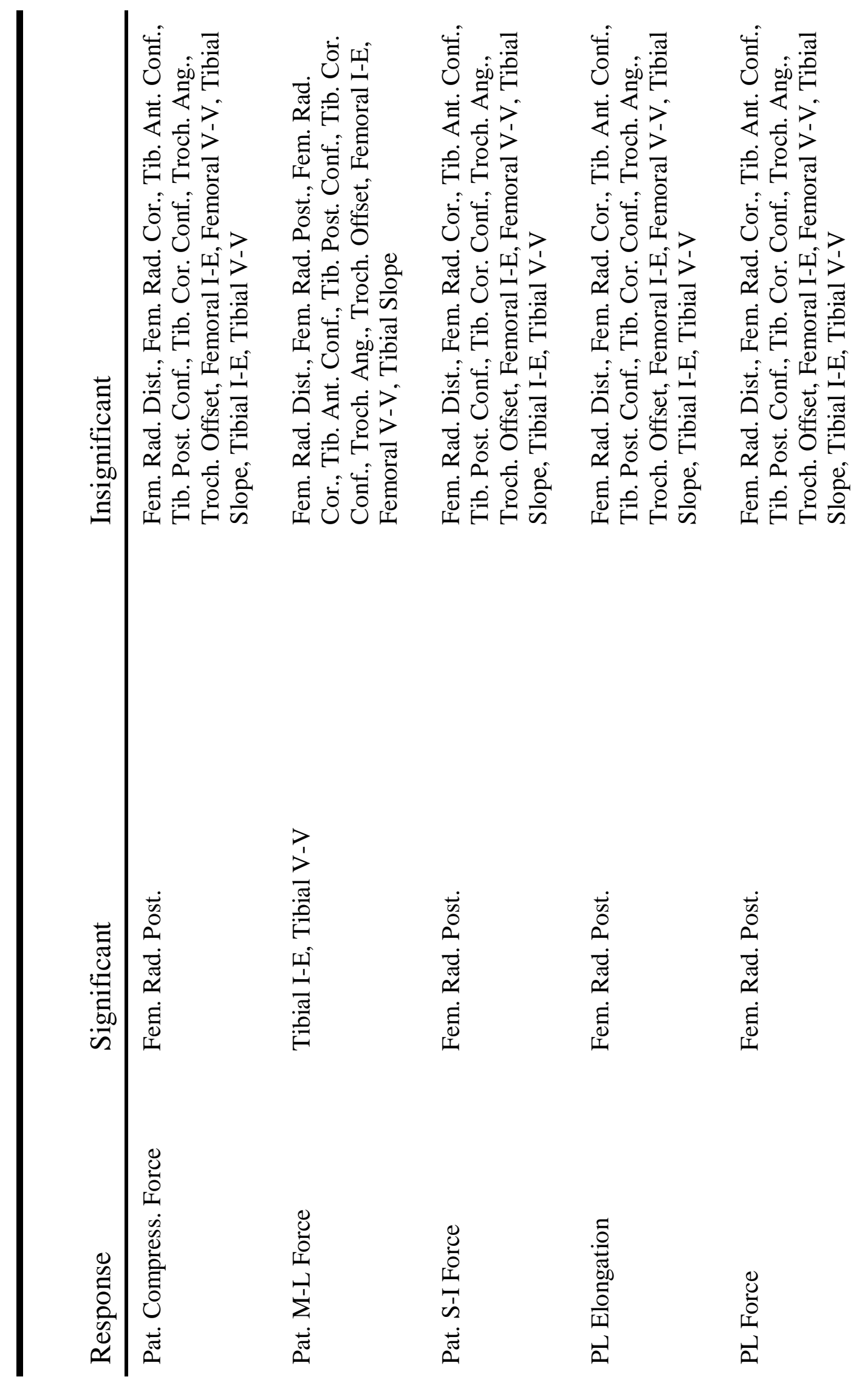




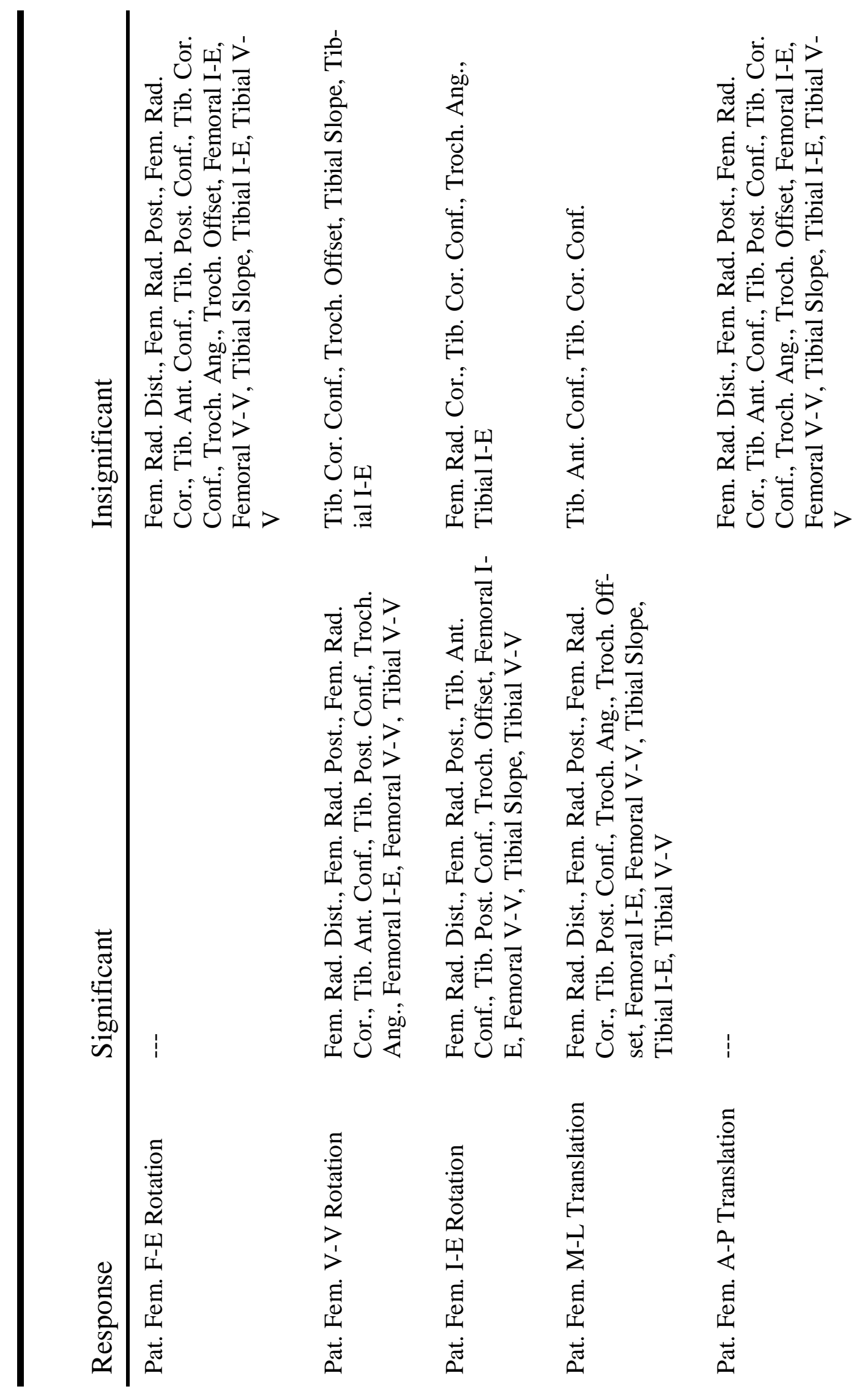




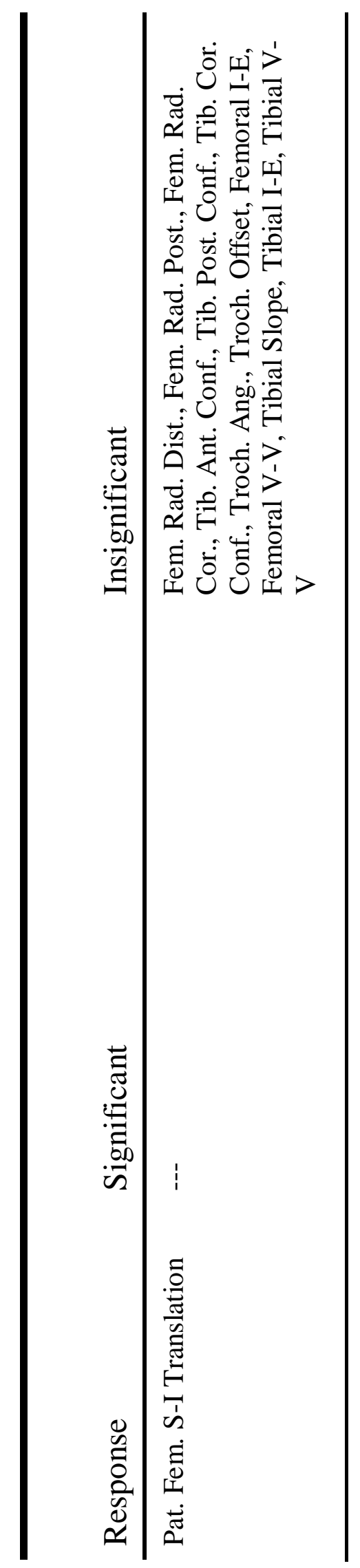




\section{APPENDIX B}

\section{DESIGN SET - TWO SAMPLE T-TEST FACTOR SENSITIVITIES}

The following charts and were produced using the design set with linear predictors and a sample rate of 20 simulations per parameter. For all t-tests, $\alpha=0.05$. The cam radius parameter was removed when moving from the design to the combination parameter sets, and the response variables for which it was significant is included (Table 14). 
Table 14. Significance of response variables for the Cam Radius parameter. This parameter was removed from the combined factor data set.

\begin{tabular}{|c|c|c|}
\hline Group & Significant & Insignificant \\
\hline TF Cont. Mech. & $\begin{array}{l}\text { Med. M-L Cntr. of Press., Med. } \\
\text { S-I Cntr. of Press., Lat. S-I Cntr. } \\
\text { of Press. }\end{array}$ & $\begin{array}{l}\text { Med. Cont. Area, Lat. Cont. } \\
\text { Area, Med. A-P Cntr. of Press., } \\
\text { Lat. M-L Cntr. of Press., Lat. A- } \\
\text { P Cntr. of Press., Med. Cont. } \\
\text { Press., Lat. Cont. Press. }\end{array}$ \\
\hline TF Jnt. Loads & $\begin{array}{l}\text { Med. I-E Torque, Med. F-E } \\
\text { Torque }\end{array}$ & $\begin{array}{l}\text { Med. Compress. Force, Med. A- } \\
\text { P Force, Lat. Compress. Force, } \\
\text { Lat. A-P Force, Lat. I-E Torque, } \\
\text { Lat. F-E Torque }\end{array}$ \\
\hline TF Kinematics & --- & $\begin{array}{l}\text { Tib. Fem. V-V Rotation, Tib. } \\
\text { Fem. I-E Rotation, Tib. Fem. } \\
\text { M-L Translation, Tib. Fem. A-P } \\
\text { Translation, Tib. Fem. S-I } \\
\text { Translation }\end{array}$ \\
\hline TF Ligaments & --- & $\begin{array}{l}\text { AP Elongation, MCL Elonga- } \\
\text { tion, MCLA Elongation, MCLP } \\
\text { Elongation, LCL Elongation, } \\
\text { LCLA Elongation, LCLP Elon- } \\
\text { gation, PFL Elongation, PFL } \\
\text { Force, ALC Elongation, PCM } \\
\text { Elongation, PCL Elongation, } \\
\text { POL Elongation, POM Elonga- } \\
\text { tion, PMC Elongation, VI Elon- } \\
\text { gation, VI Force }\end{array}$ \\
\hline
\end{tabular}




\begin{tabular}{lll} 
Group & Significant & Insignificant \\
\hline Patellofemoral & --- & Pat. Cont. Area, Pat. M-L Cntr. \\
& of Press., Pat. S-I Cntr. of \\
& Press., Pat. A-P Cntr. of Press., \\
& Pat. Cont. Press., Pat. Com- \\
& press. Force, Pat. M-L Force, \\
& Pat. S-I Force, PL Elongation, \\
& PL Force, Pat. Fem. F-E Rota- \\
& tion, Pat. Fem. V-V Rotation, \\
& Pat. Fem. I-E Rotation, Pat. \\
& Fem. M-L Translation, Pat. \\
& Fem. A-P Translation, Pat. Fem. \\
& S-I Translation \\
& \\
\hline
\end{tabular}




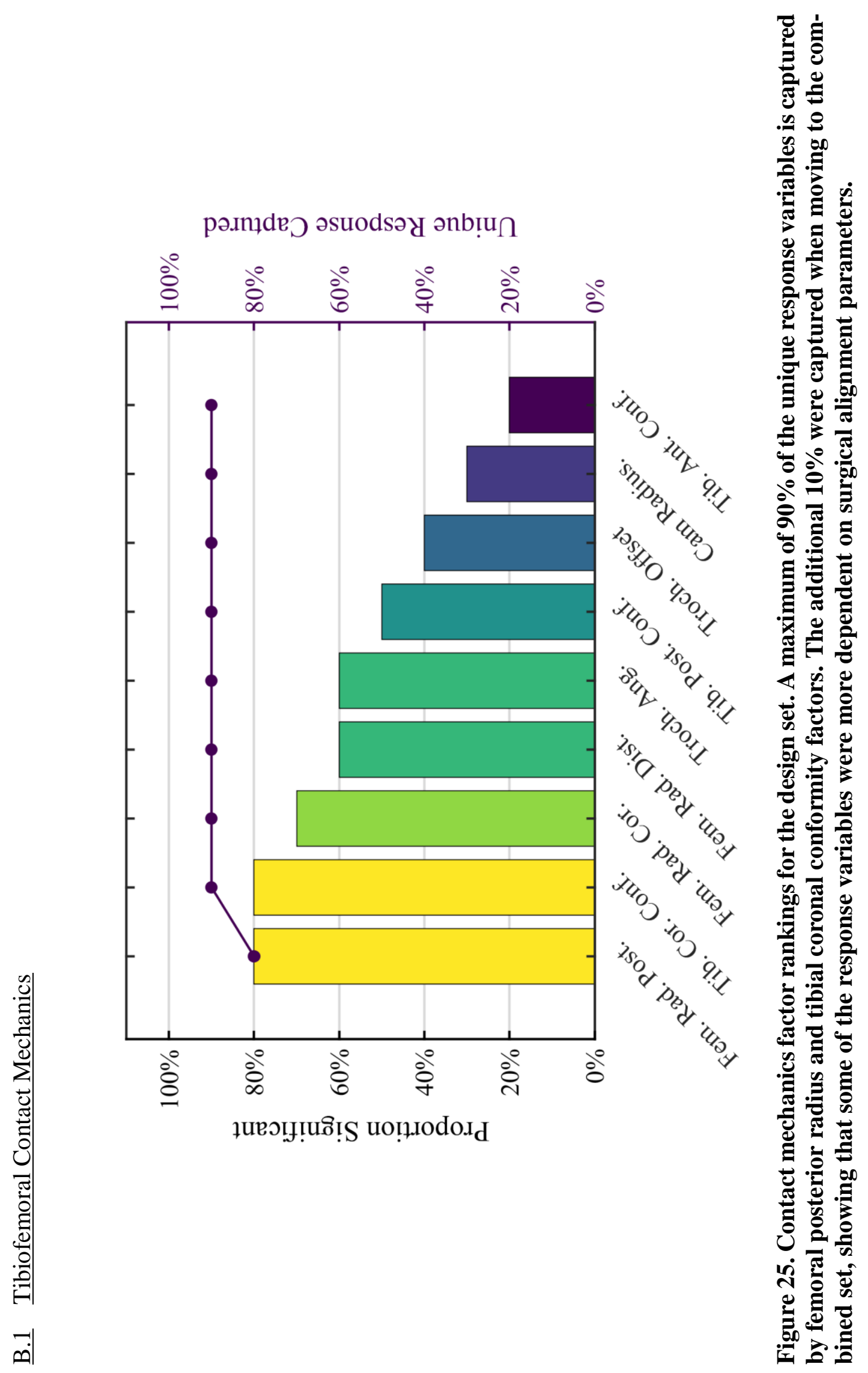




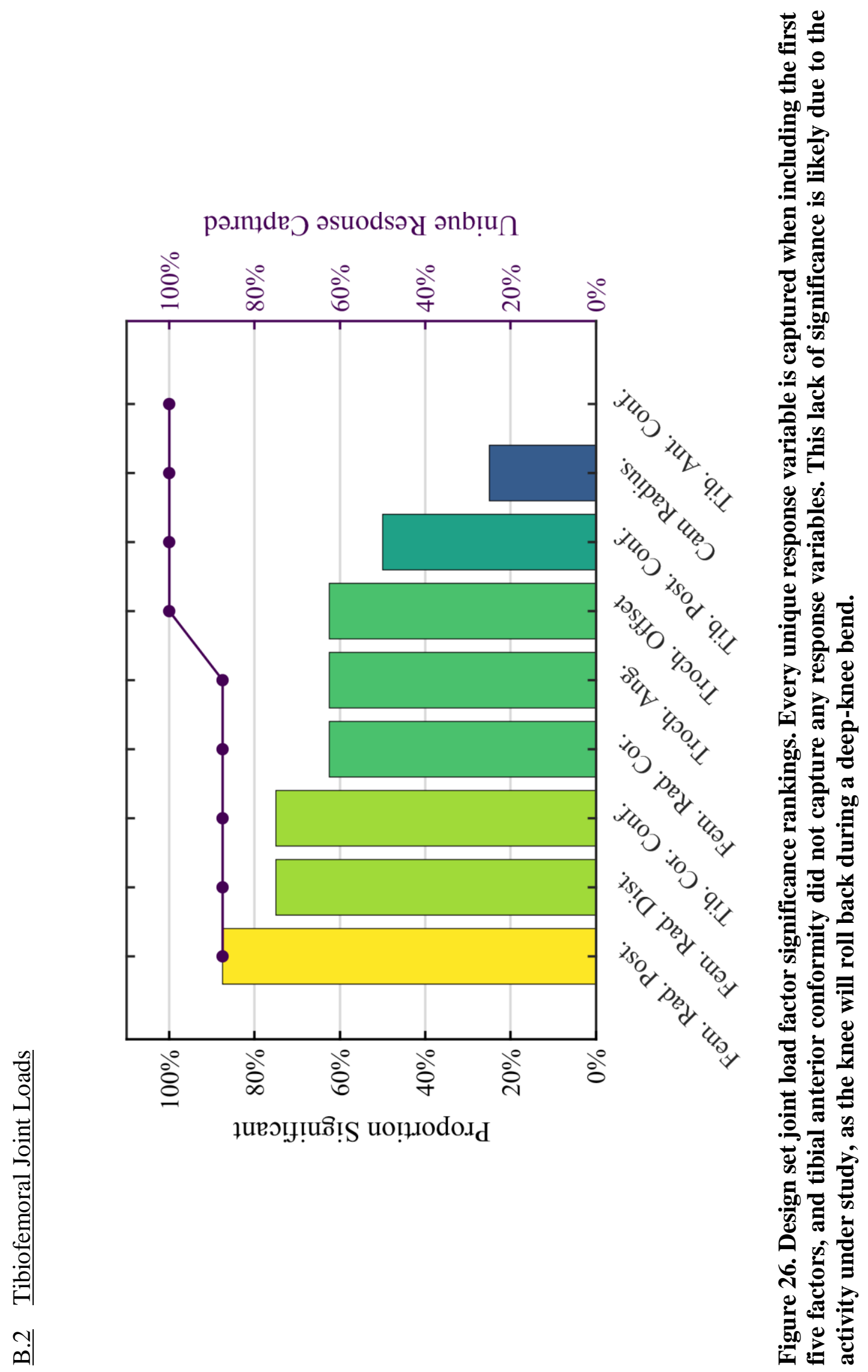




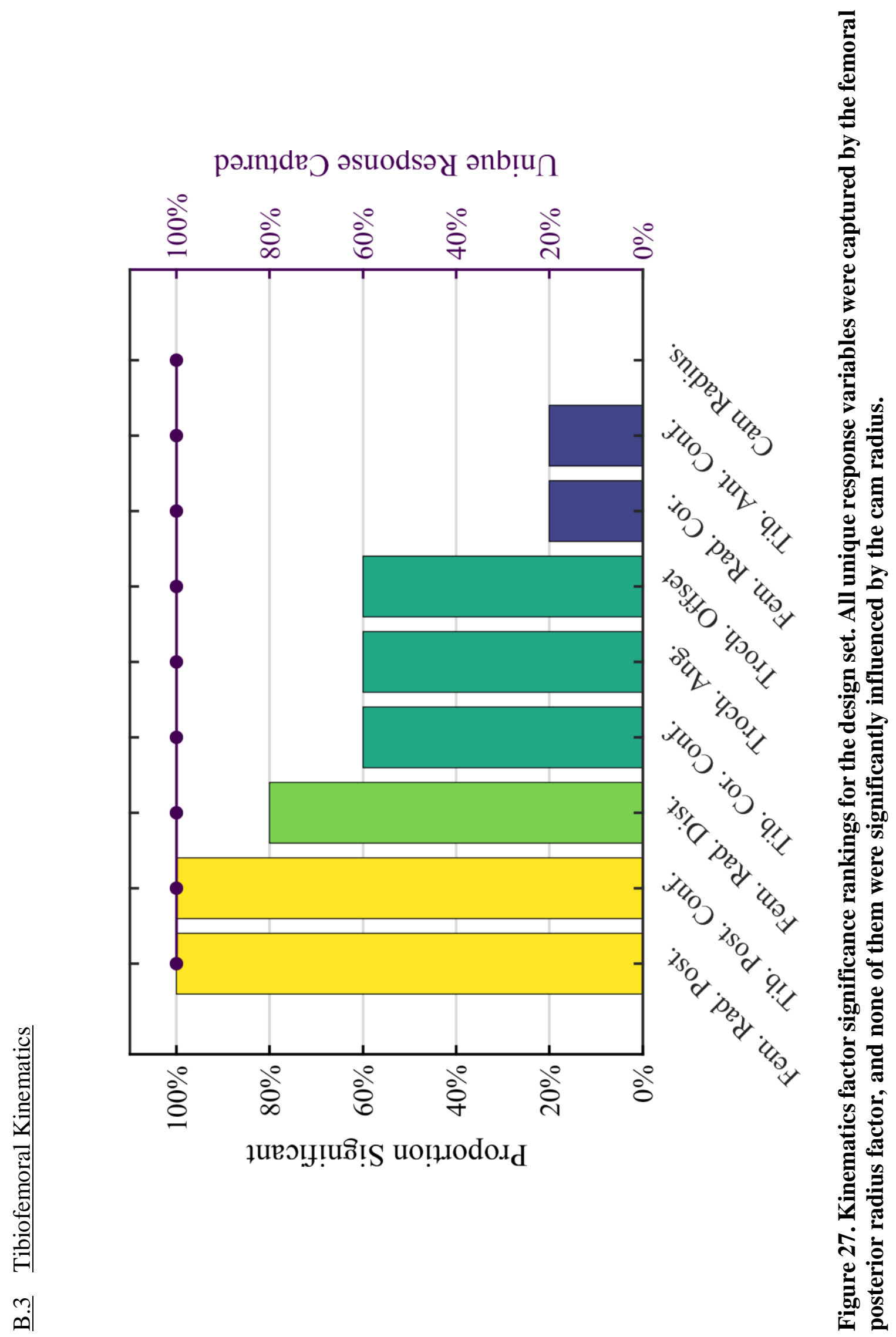




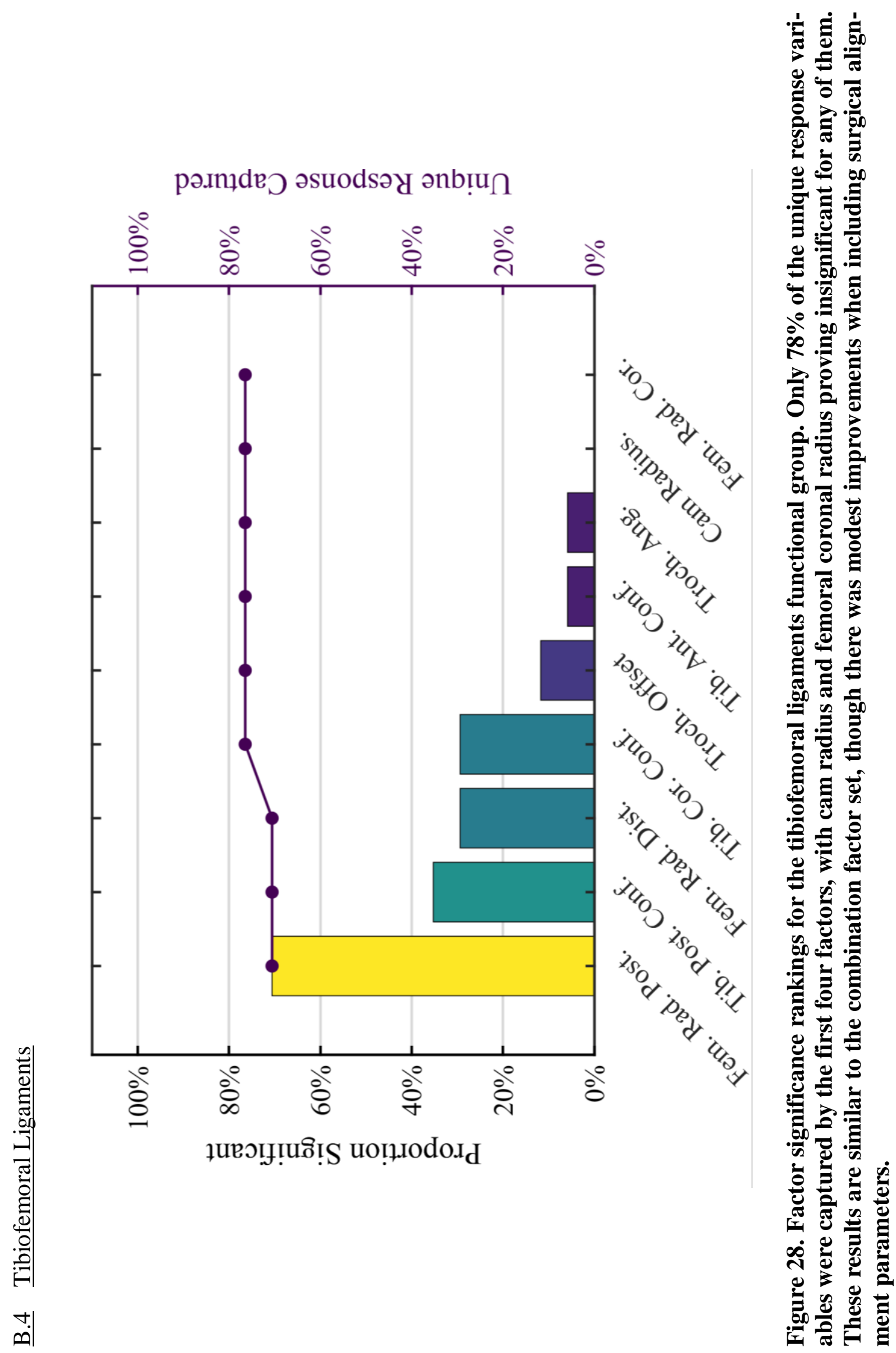




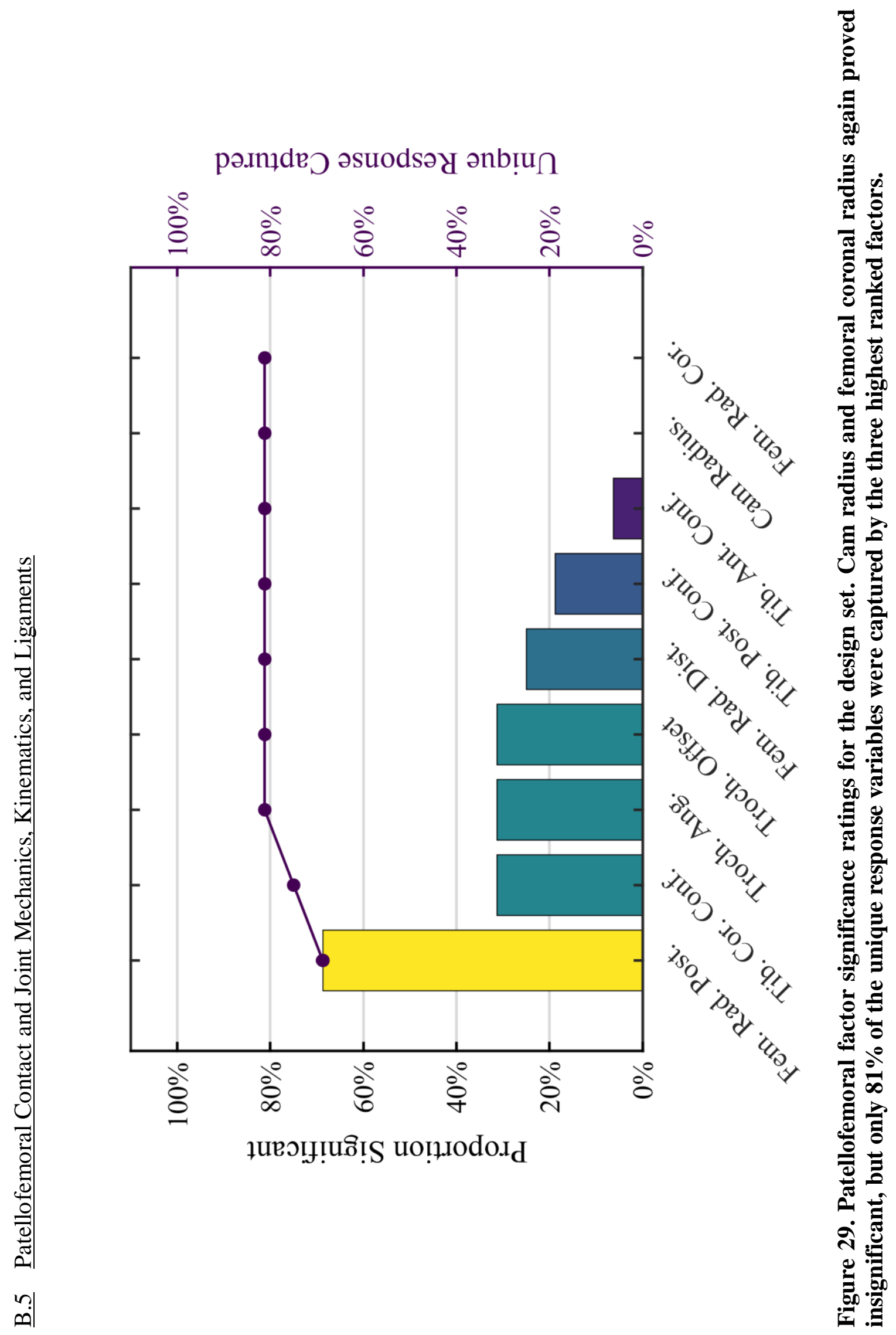




\section{APPENDIX C}

\section{SURGICAL SET - TWO SAMPLE T-TEST FACTOR SENSITIVITIES}

The following charts were produced using the surgical set with linear predictors and a sample rate of 20 simulations per parameter. For all t-tests, $\alpha=0.05$. The femoral F-E surgical alignment parameter was removed when proceeding from surgical to combined parameter sets, and the response variables for which it was significant have been tabulated (Table 15). 
Table 15. Significance of response variables for the Femoral F-E alignment parameter. This parameter was removed from the combined factor data set.

\begin{tabular}{|c|c|c|}
\hline Group & Significant & Insignificant \\
\hline TF Cont. Mech. & $\begin{array}{l}\text { Med. M-L Cntr. of Press., Lat. } \\
\text { M-L Cntr. of Press. }\end{array}$ & $\begin{array}{l}\text { Med. Cont. Area, Lat. Cont. } \\
\text { Area, Med. S-I Cntr. of Press., } \\
\text { Med. A-P Cntr. of Press., Lat. } \\
\text { S-I Cntr. of Press., Lat. A-P } \\
\text { Cntr. of Press., Med. Cont. } \\
\text { Press., Lat. Cont. Press. }\end{array}$ \\
\hline TF Jnt. Loads & $\begin{array}{l}\text { Med. Compress. Force, Med. I- } \\
\text { E Torque, Med. F-E Torque, } \\
\text { Lat. F-E Torque }\end{array}$ & $\begin{array}{l}\text { A-P Force, Lat. Compress. } \\
\text { Force, Lat. A-P Force, Lat. I-E } \\
\text { Torque }\end{array}$ \\
\hline TF Kinematics & $\begin{array}{l}\text { Tib. Fem. V-V Rotation, Tib. Fem. I- } \\
\text { E Rotation, Tib. Fem. M-L Transla- } \\
\text { tion, Tib. Fem. S-I Translation }\end{array}$ & Tib. Fem. A-P Translation \\
\hline TF Ligaments & $\begin{array}{l}\text { MCL Elongation, MCLA Elon- } \\
\text { gation, MCLP Elongation, LCL } \\
\text { Elongation, LCLA Elongation, } \\
\text { LCLP Elongation, PMC Elon- } \\
\text { gation }\end{array}$ & $\begin{array}{l}\text { AP Elongation, PFL Elongation, } \\
\text { PFL Force, ALC Elongation, } \\
\text { PCM Elongation, PCL Elonga- } \\
\text { tion, POL Elongation, POM } \\
\text { Elongation, VI Elongation, VI } \\
\text { Force }\end{array}$ \\
\hline Patellofemoral & $\begin{array}{l}\text { Pat. M-L Cntr. of Press., Pat. } \\
\text { Fem. V-V Rotation, Pat. Fem. } \\
\text { A-P Translation }\end{array}$ & $\begin{array}{l}\text { Pat. Cont. Area, Pat. S-I Cntr. of } \\
\text { Press., Pat. A-P Cntr. of Press., } \\
\text { Pat. Cont. Press., Pat. Com- } \\
\text { press. Force, Pat. M-L Force, } \\
\text { Pat. S-I Force, PL Elongation, } \\
\text { PL Force, Pat. Fem. F-E Rota- } \\
\text { tion, Pat. Fem. I-E Rotation, } \\
\text { Pat. Fem. M-L Translation, Pat. } \\
\text { Fem. S-I Translation }\end{array}$ \\
\hline
\end{tabular}




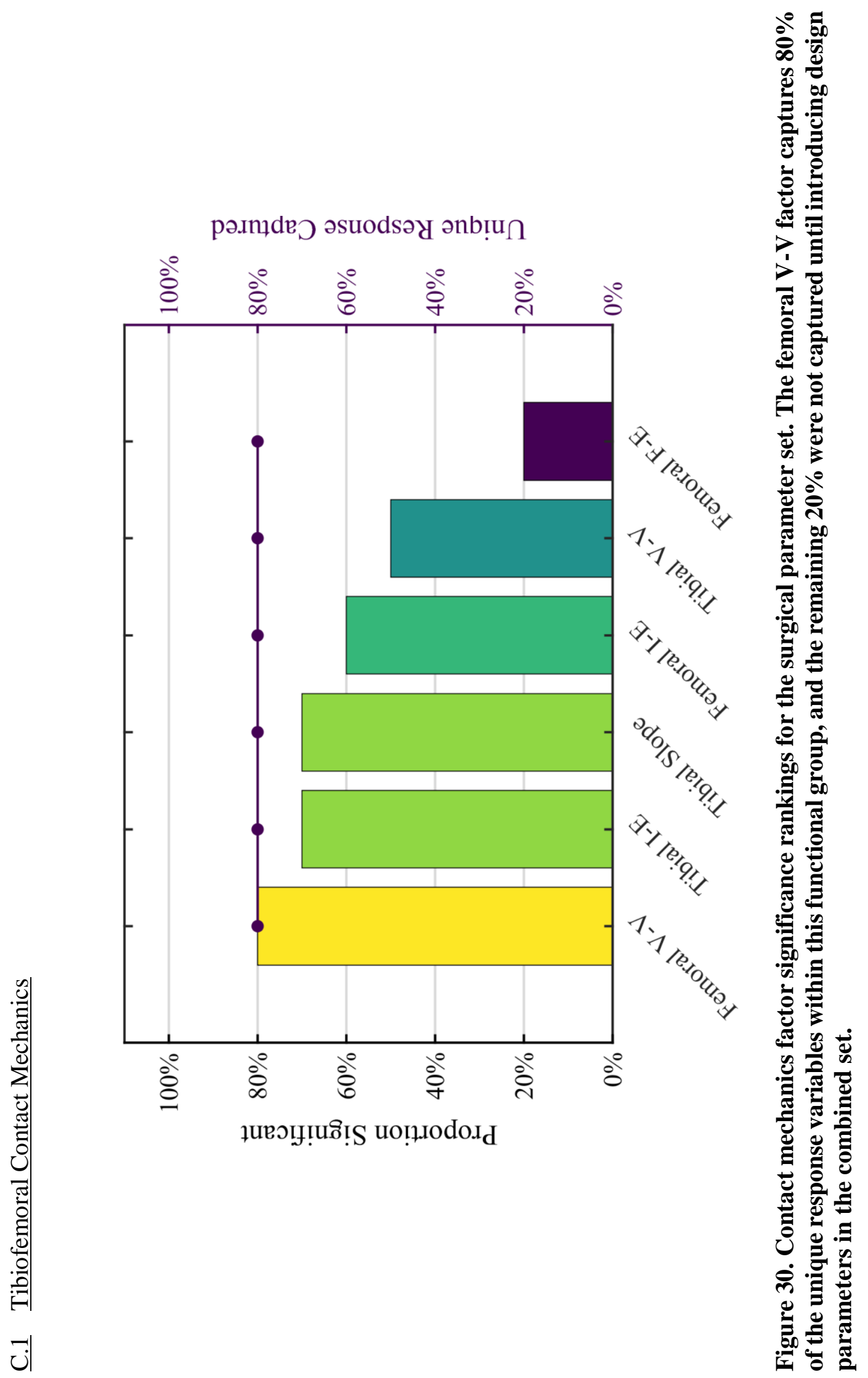




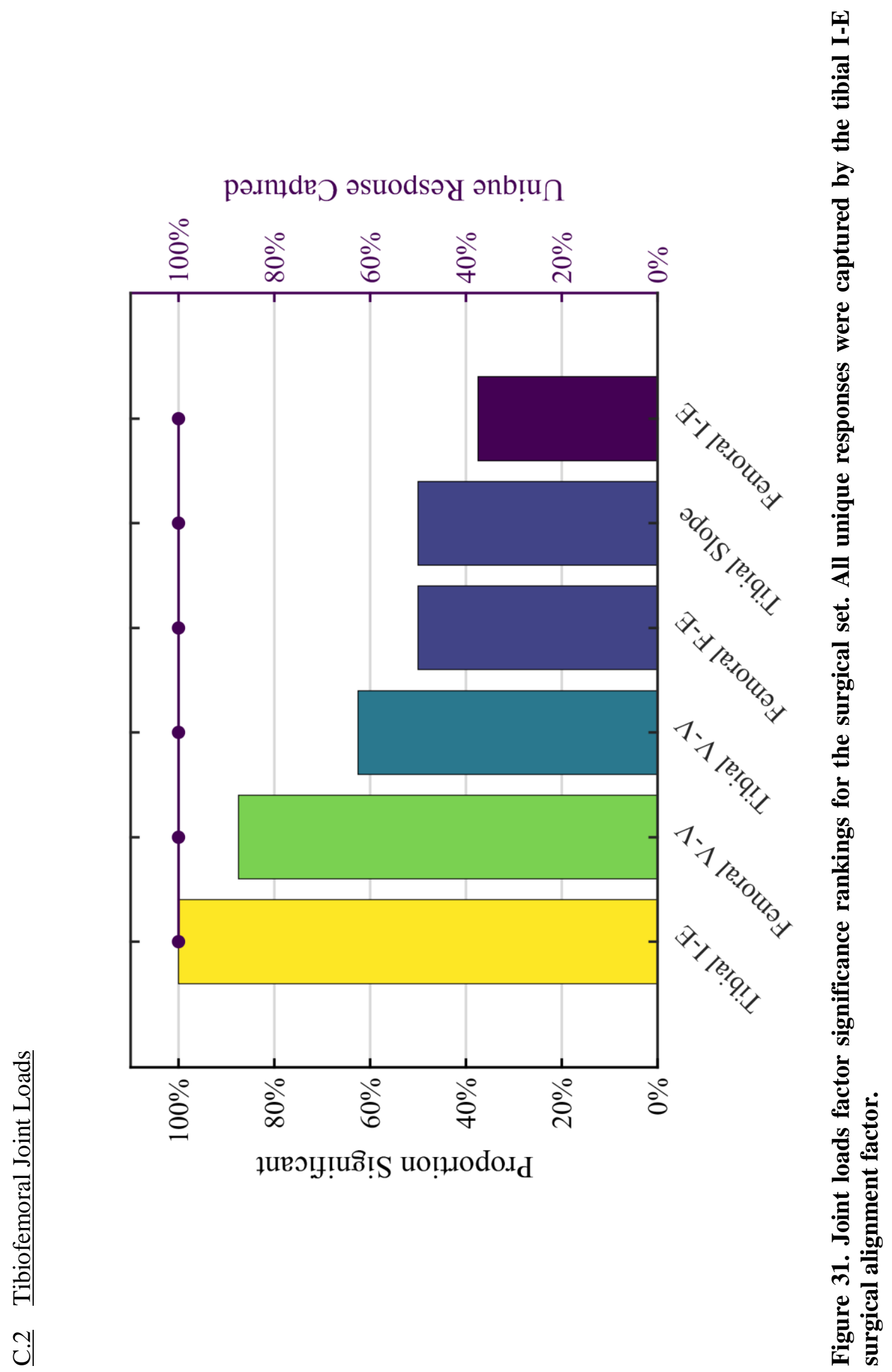




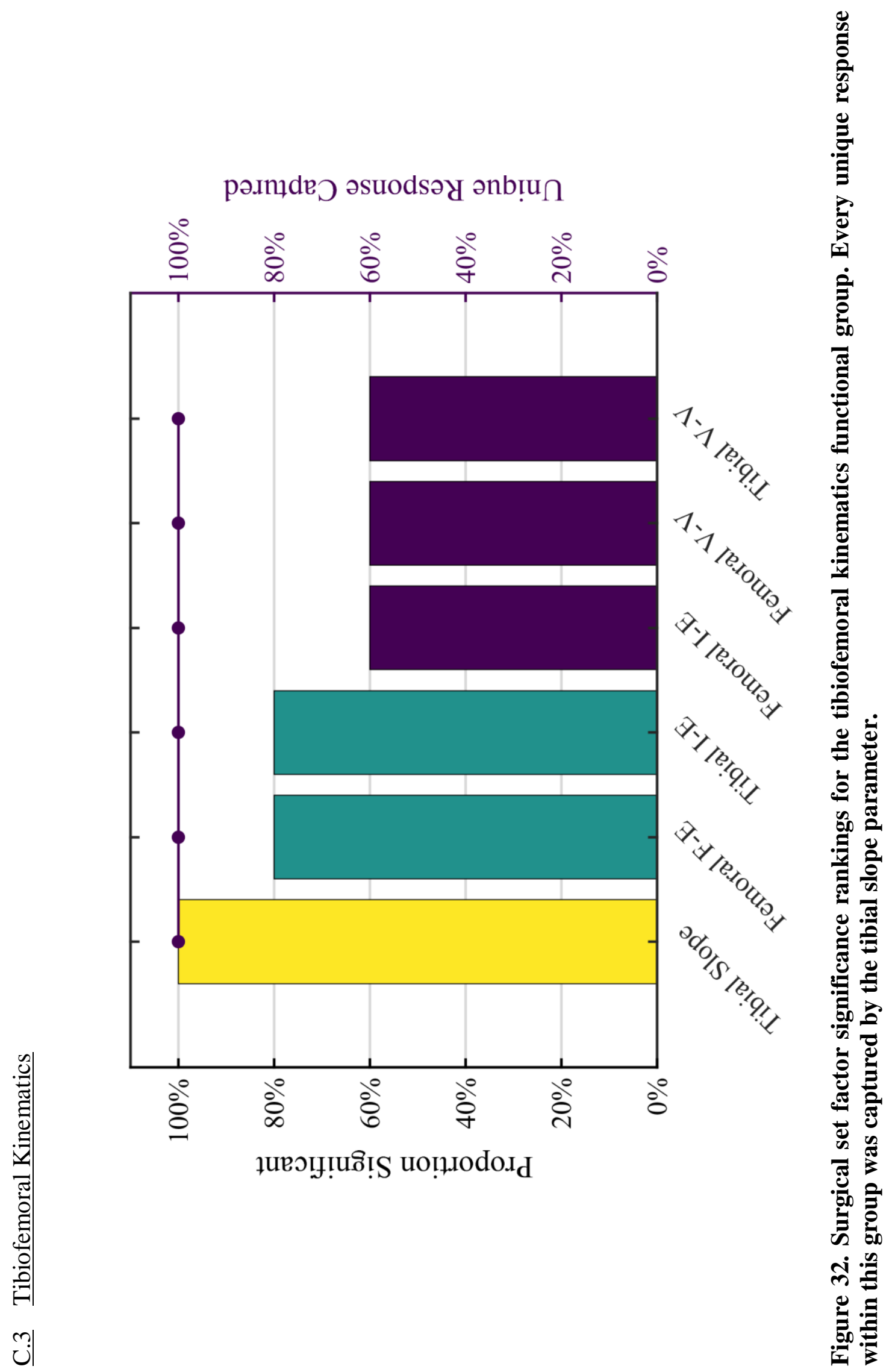




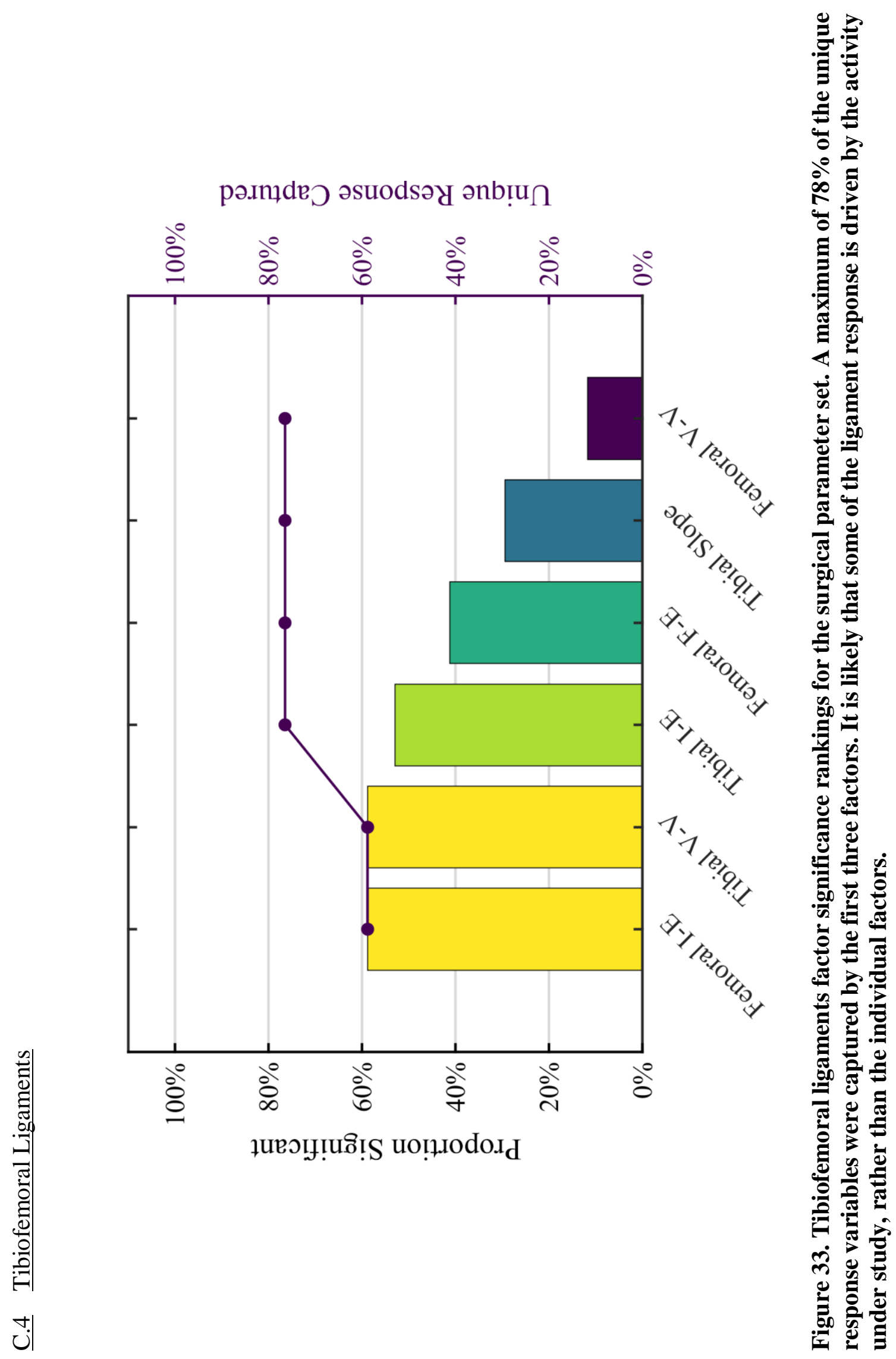




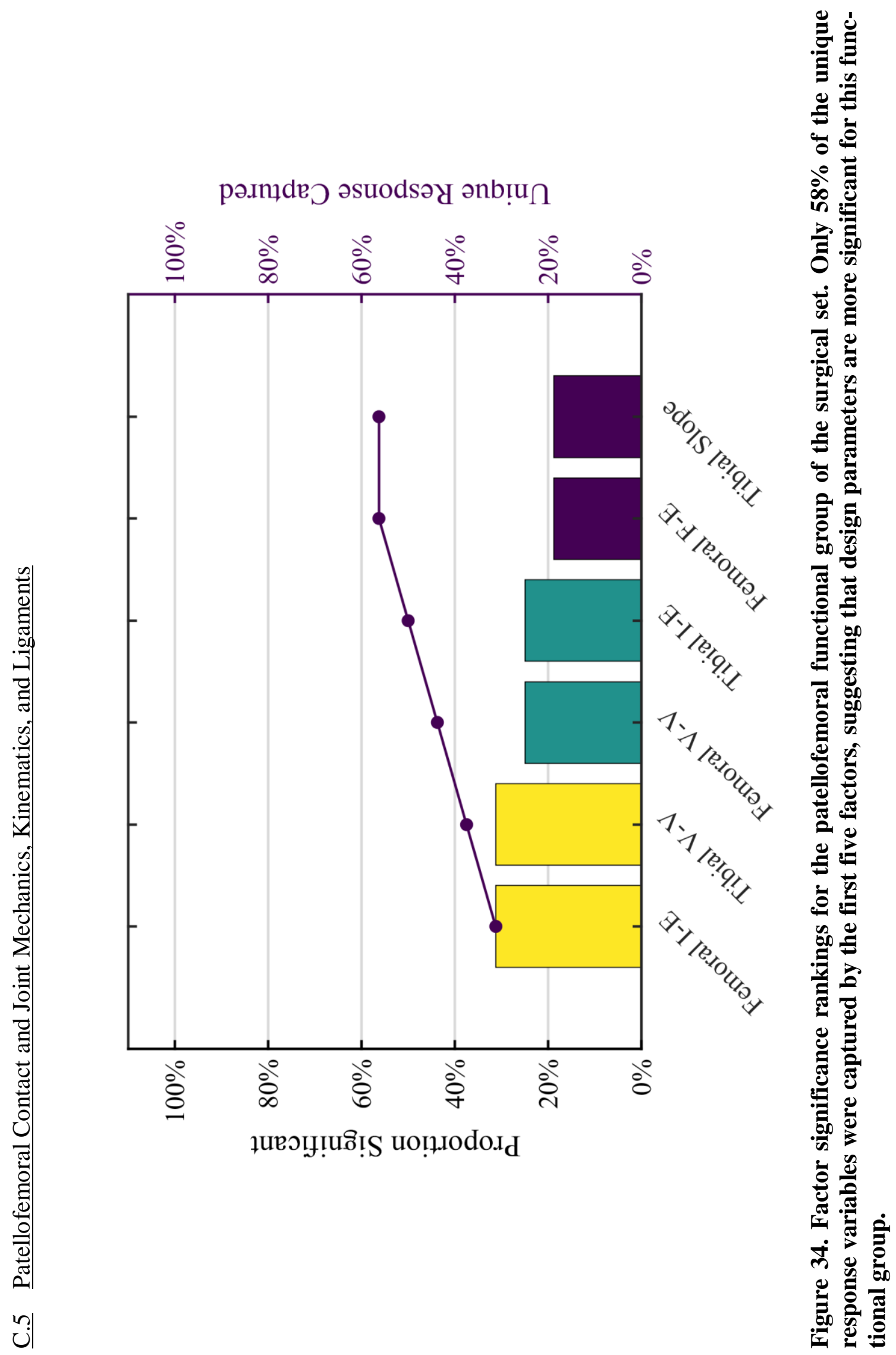

